\title{
Interactions in Collaborative Prototyping for Early Design Development in Pediatric Medication Safety
}

\author{
by \\ Catherine Campbell
}

A thesis submitted to the Faculty of Graduate and Postdoctoral Affairs in partial fulfillment of the requirements for the degree of

Master of Design

in

Industrial Design

Carleton University

Ottawa, Ontario

(C) 2010, Catherine Campbell 


$\begin{array}{ll}\begin{array}{l}\text { Library and Archives } \\ \text { Canada }\end{array} & \begin{array}{l}\text { Bibliothèque et } \\ \text { Archives Canada }\end{array} \\ \begin{array}{l}\text { Published Heritage } \\ \text { Branch }\end{array} & \begin{array}{l}\text { Direction du } \\ \text { Patrimoine de l'édition }\end{array} \\ \begin{array}{l}\text { 395 Wellington Street } \\ \text { Ottawa ON K1A ON4 } \\ \text { Canada }\end{array} & \begin{array}{l}395, \text { rue Wellington } \\ \text { Ottawa ON K1A ON4 } \\ \text { Canada }\end{array}\end{array}$

Your file Votre référence ISBN: 978-0-494-71537-6 Our file Notre référence ISBN: 978-0-494-71537-6

NOTICE:

The author has granted a nonexclusive license allowing Library and Archives Canada to reproduce, publish, archive, preserve, conserve, communicate to the public by telecommunication or on the Internet, loan, distribute and sell theses worldwide, for commercial or noncommercial purposes, in microform, paper, electronic and/or any other formats.

The author retains copyright ownership and moral rights in this thesis. Neither the thesis nor substantial extracts from it may be printed or otherwise reproduced without the author's permission.
AVIS:

L'auteur a accordé une licence non exclusive permettant à la Bibliothèque et Archives Canada de reproduire, publier, archiver, sauvegarder, conserver, transmettre au public par télécommunication ou par l'Internet, prêter, distribuer et vendre des thèses partout dans le monde, à des fins commerciales ou autres, sur support microforme, papier, électronique et/ou autres formats.

L'auteur conserve la propriété du droit d'auteur et des droits moraux qui protège cette thèse. $\mathrm{Ni}$ la thèse ni des extraits substantiels de celle-ci ne doivent être imprimés ou autrement reproduits sans son autorisation.
In compliance with the Canadian Privacy Act some supporting forms may have been removed from this thesis.

While these forms may be included in the document page count, their removal does not represent any loss of content from the thesis.
Conformément à la loi canadienne sur la protection de la vie privée, quelques formulaires secondaires ont été enlevés de cette thèse.

Bien que ces formulaires aient inclus dans la pagination, il n'y aura aucun contenu manquant.

\section{Canadä}


INTERACTIONS IN COLLABORATIVE PROTOTYPING

\begin{abstract}
Prototyping is an established tool for communication between stakeholders in design development. Recent studies have focused on the development of methods for early collaborative prototyping supporting communication and concept development in interdisciplinary design teams. The Canadian healthcare system is facing complex challenges demanding collaboration between experts from multiple disciplines and could potentially benefit from the application of such methods. Thus this research aimed to explore the use of cross-functional collaborative prototyping (CFCPing) to support communication between disciplines through a focused study addressing medication safety in a pediatric hospital. A comparison of verbal and physical behaviours exhibited across participating disciplines suggested that designers and non-designers assumed different roles and that groups exhibiting frequent transitions between ideation and evaluation generated more ideas than the groups exhibiting comparatively frequent transitions between information and management type behaviours. Implications for the conduct of future CFCPing sessions, applications in healthcare and future research are discussed.
\end{abstract}

Keywords: interdisciplinary, design development, collaborative prototyping, medication room, pediatric hospital 
INTERACTIONS IN COLLABORATIVE PROTOTYPING

\section{Acknowledgements}

I would first like to thank my advisors, Prof. Thomas Garvey at the School of Industrial Design and Prof. Avi Parush from the Department of Psychology for their continued support, guidance and availability to discuss my thoughts and ideas when developing and executing this project. I am also grateful to Prof. WonJoon Chung, the original author of the CFCPing method, for many conversations at the outset of this research and for participating as a member of my examination committee. Furthermore, I would like to thank Prof. Adrian Chan from the Department of Systems and Computer Engineering for his valuable insight and feedback as a member of my examination committee.

Dr. Jacqueline Ellis, Dr. Régis Vaillancourt and the rest of the IV Morphine Study team were a pleasure to work with and provided invaluable support throughout this project. I am also grateful to the nurses, pharmacists, pharmacy technicians and professional industrial designers who participated in the collaborative sessions. Furthermore, this study would not have been possible without funding from MITACS and CHEO.

Finally, I would like to thank my family, friends and colleagues in the Master of Design program at Carleton for their continued support and encouragement throughout this process. 


\section{Table of Contents}

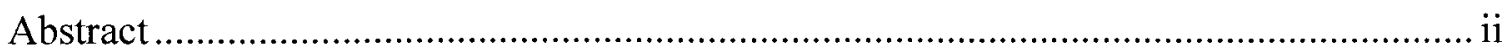

Acknowledgements ..................................................................................................

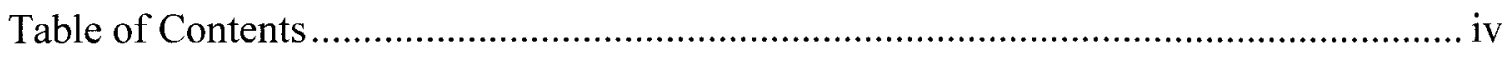

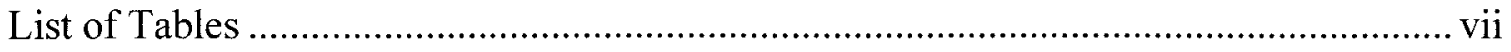

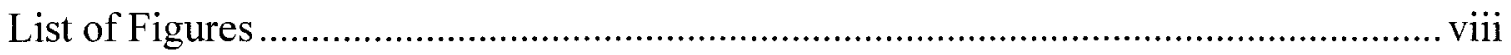

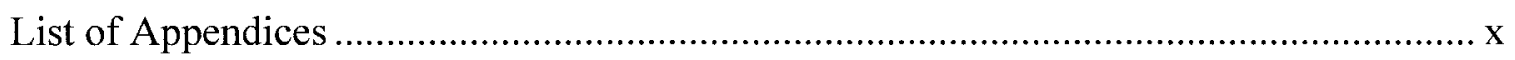

Glossary of Terms ............................................................................................... xi

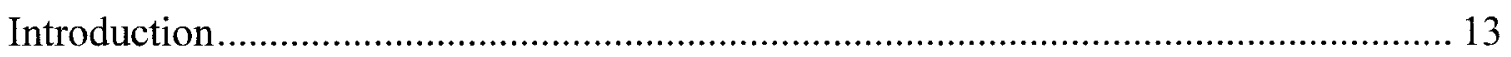

Collaborative Prototyping for Early Concept Development ......................................... 13

An Opportunity for Empirical Research ……………...................................... 14

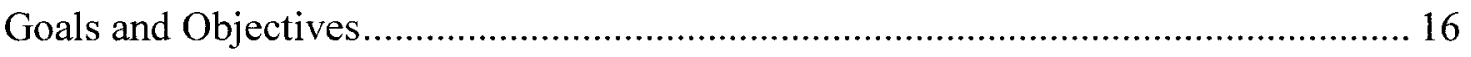

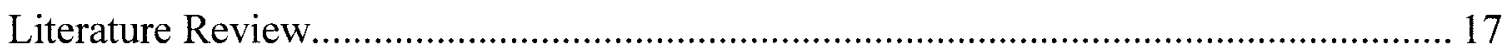

Concept Development at the Fuzzy-Front-End....................................................... 17

Brainstorming and Creative Problem Solving in Small Groups ................................. 18

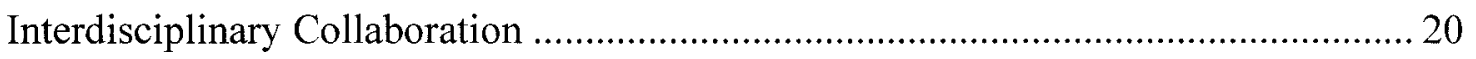

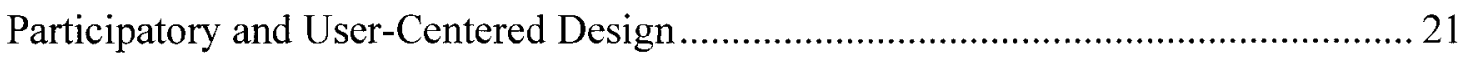

Knowledge Management in Interdisciplinary Collaboration ........................................2 25

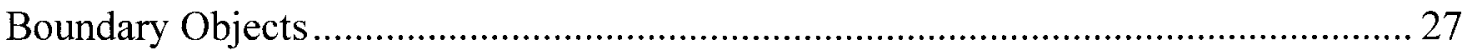

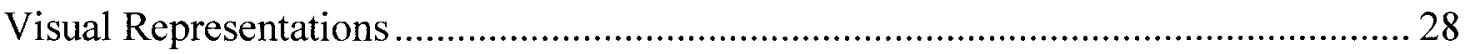

Cross-Functional Collaborative Prototyping (CFCPing) ……………………............. 31

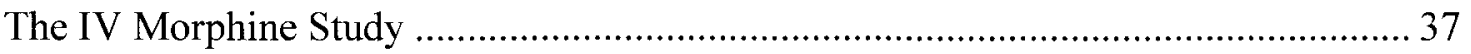




\section{INTERACTIONS IN COLLABORATIVE PROTOTYPING}

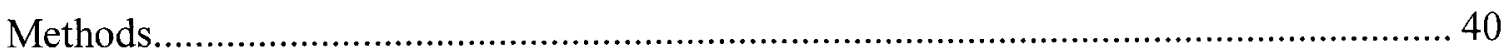

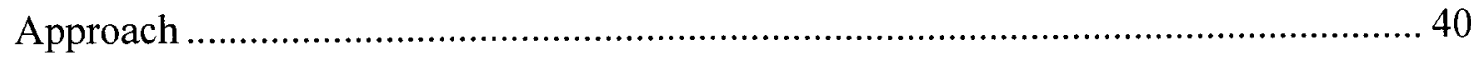

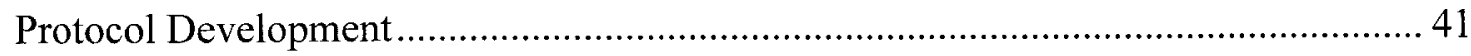

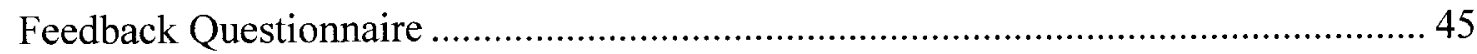

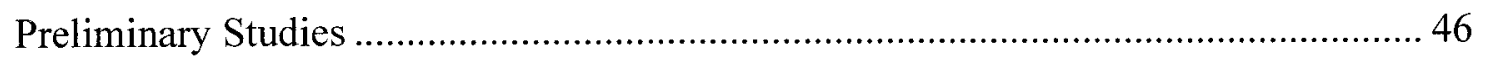

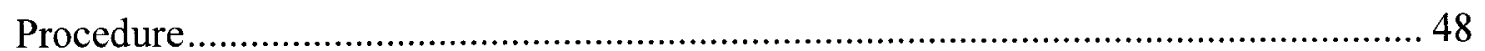

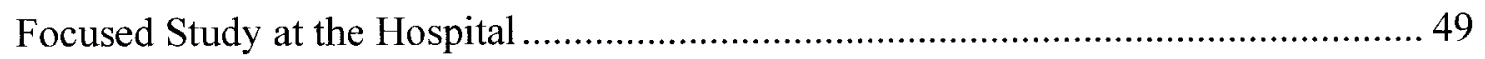

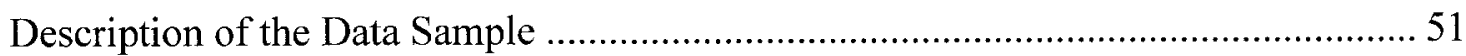

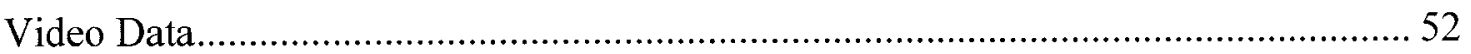

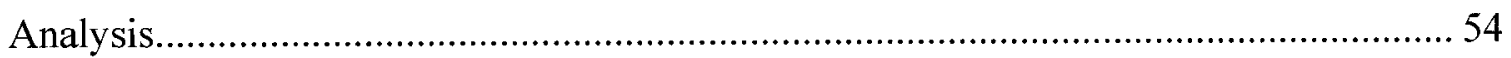

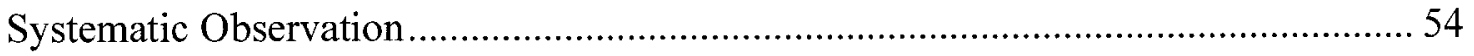

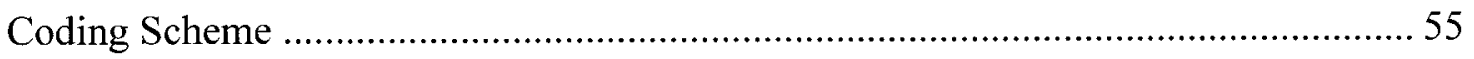

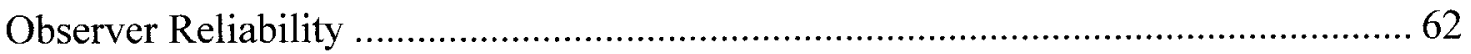

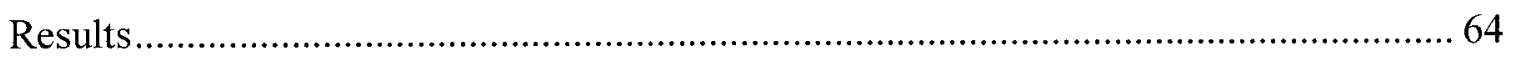

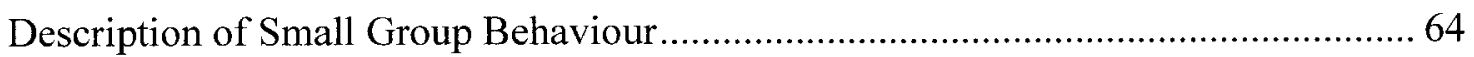

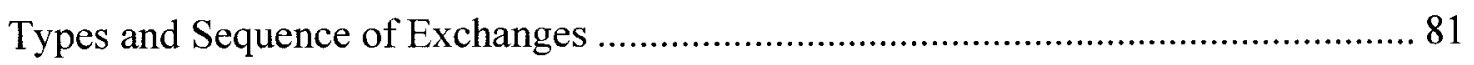

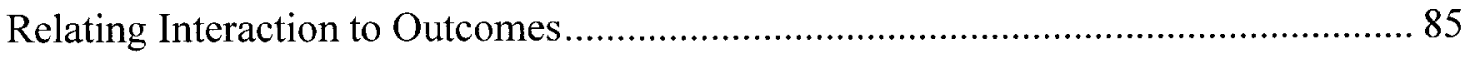

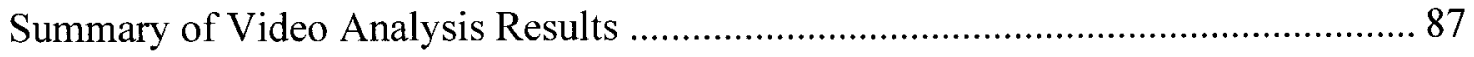

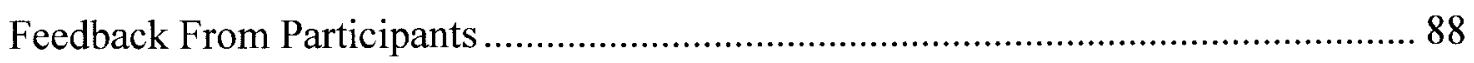

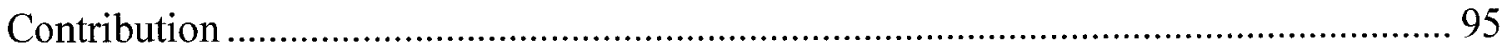

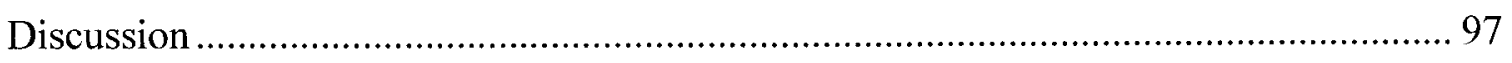

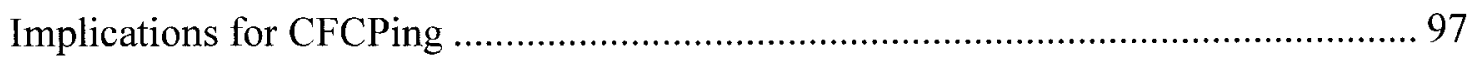

Implications for use of CFCPing in Healthcare Settings .................................. 102 


\section{INTERACTIONS IN COLLABORATIVE PROTOTYPING}

Limitations of the Study ...................................................................................... 104

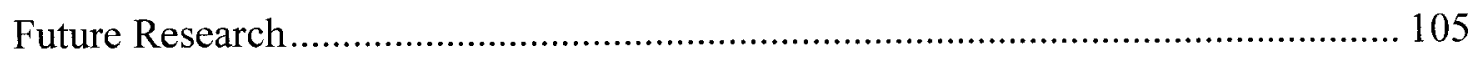

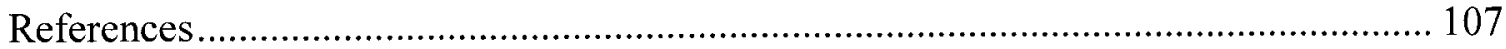




\section{INTERACTIONS IN COLLABORATIVE PROTOTYPING}

\section{List of Tables}

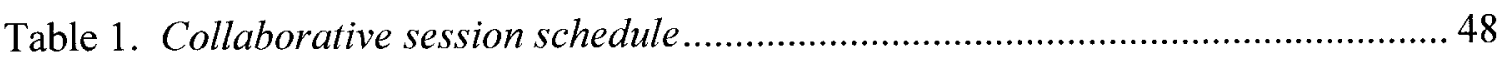

Table 2. Coding scheme to identify individual behaviours ........................................ 57

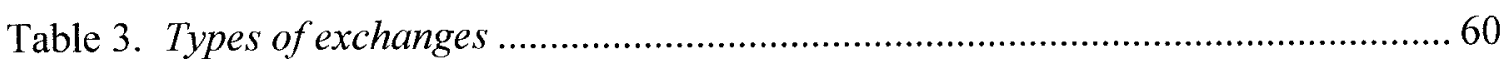

Table 4. Example of coded exchanges in one minute of dialogue ................................ 61

Table 5. Example dialogue including explanatory behaviour .................................... 66

Table 6. Example dialogue including explanatory behaviour ..................................... 66

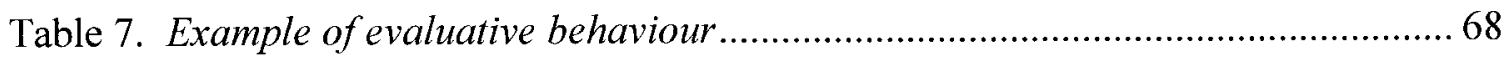

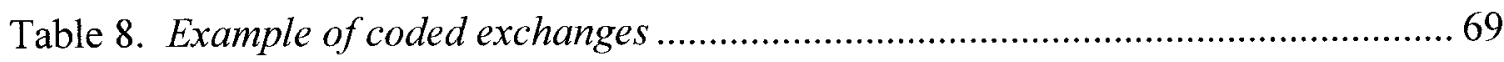

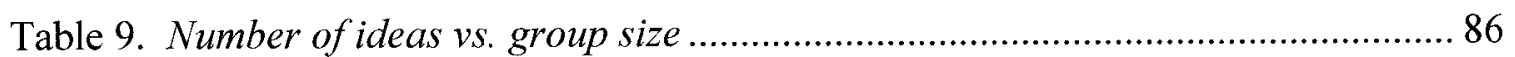

Table 10. Example of preliminary interaction analysis for collaborative session \#1 ... 131

Table 11. Example dialogue showing information exchange (verbal behaviour only) 133

Table 12. Example dialogue showing information exchange (verbal \& physical) ....... 133

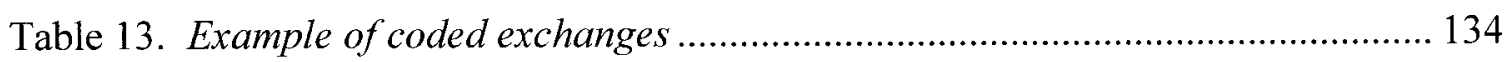

Table 14. Example dialogue showing suggestion and evaluation exchanges .............. 134 
INTERACTIONS IN COLLABORATIVE PROTOTYPING

List of Figures

Figure 1. CFCPing Method by WonJoon Chung (2009, p. 116) ................................ 35

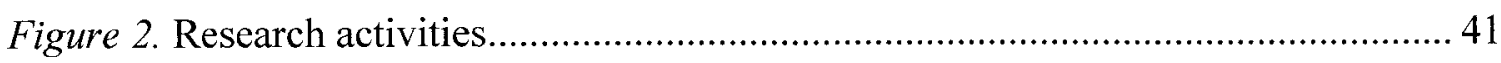

Figure 3. Collaborative session structure ......................................................... 42

Figure 4. Breakdown of participants in each group by discipline ...............................52

Figure 5. Example of general observations capturing small group behaviour ............... 63

Figure 6. Average proportion of information-seeking behaviour (question and request

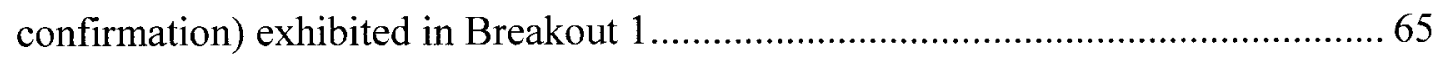

Figure 7. Average number of evaluative behaviours exhibited in Breakout 1 ................. 68

Figure 8. Average frequency of physical behaviours exhibited by discipline ............... 71

Figure 9. Using reference images and gesture to support information transfer .............. 72

Figure 10. Examples of sketching and interaction with visual representations .............. 73

Figure 11. Nurse makes model of existing MAR to explain concept to designer........... 75

Figure 12. Pharmacy technician uses a piece of the model to generate new ideas .......... 75

Figure 13. Designer makes new elements of the model............................................ 76

Figure 14. Relative participation by discipline in Breakout 1 ...................................... 77

Figure 15. A group of five self-organizes into two smaller groups.............................. 78

Figure 16. Interactions observed in a five-person group........................................ 79

Figure 17. Proportion of group activity as a function of exchange type ....................... 82

Figure 18. Emergent concept development Process 1 exhibited by CS1G1 \& CS2G2 ... 84

Figure 19. Emergent concept development Process 2 exhibited by CS1G2 \& CS1G3 ... 85

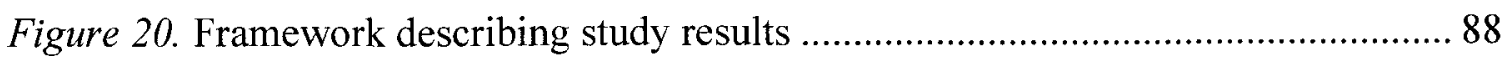

Figure 21. Feedback from participants on the Pictionary warm-up game ..................... 90 


\section{INTERACTIONS IN COLLABORATIVE PROTOTYPING}

Figure 22. Feedback from participants on the use of sketching and prototyping............ 90

Figure 23. Feedback regarding the modification of prototypes to share ideas................ 91

Figure 24. Participant perception of sketching and prototyping ................................. 92

Figure 25. Example of a coded video clip........................................................ 132 
INTERACTIONS IN COLLABORATIVE PROTOTYPING

\section{List of Appendices}

Appendix 1. Collaborative Design Session Protocol ......................................... 117

Appendix 2. Collaborative Session Feedback Survey …..................................... 124

Appendix 3. Collaborative Session Introductory Presentation ............................... 127

Appendix 4. Behaviour Analysis by Discipline for Individual Groups.................... 131

Appendix 5. Examples of Coded Dialogue........................................................ 132 
INTERACTIONS IN COLLABORATIVE PROTOTYPING

\section{Glossary of Terms}

Adverse events (AEs): Defined as "undesired and unplanned occurrences, directly associated with the care or services provided to a patient/client in the health care system" (The Royal College of Physicians and Surgeons of Canada [RCPSC], 2003).

Boundary objects: Originally defined by Star \& Greisemer (1989) as the notion that artifacts can occupy multiple intersecting worlds and satisfy information requirements in each of the. In product design and knowledge management circles these are physical objects that support the transfer, translation and transformation of knowledge between collaborating disciplines (Carlile, 2002).

Collaborative prototyping: in the context of this study, collaborative prototyping refers to an interdisciplinary team of designers and non-designers making prototypes and mockups of design ideas during brainstorming and early concept development. These mockups serve as boundary objects and scaffolding (Chung, 2009) supporting knowledge management and ideation.

Generative tools: refers to "the creation of a shared design language that designers/ researchers and the stakeholders use to communicate visually and directly with each other... with it, people can express an infinite number of ideas through a limited set of stimulus items" (Sanders \& Chan, 2007). This includes specifically designed or selected sets of prototyping materials that help users (usually non-designers) visually represent their ideas.

Interdisciplinary collaboration: problem solving involving individuals from a variety of educational and/or professional backgrounds and experience working together, towards a 
INTERACTIONS IN COLLABORATIVE PROTOTYPING

shared goal. In the context of this study, the objective of collaborative efforts is the development of innovative products and service concepts.

Interprofessional care: (or Interdisciplinary care) is defined by multiple health caregivers working collaboratively to deliver quality patient care within and across settings (Health Force Ontario, 2007).

Medical error: the term used to represent "the failure to complete a planned action as it was intended or when an incorrect plan is used in an attempt to achieve a given aim" (RCPSC, 2003).

Medication error: a medical error that occurs "at any point in the process of providing medications to patients" (RCPSC, 2003).

Participatory design: a cooperative approach to design development where professional designers work with experienced users "acting as lay designers" (Bødker et al., 1988, p. $385)$.

Patient safety: "the reduction and mitigation of unsafe acts within the health-care system, as well as through the use of best practices shown to lead to optimal patient outcomes" (RCPSC, 2003). 
INTERACTIONS IN COLLABORATIVE PROTOTYPING

Introduction

\section{Collaborative Prototyping for Early Concept Development}

In a world where interrelationships between technology, people and products is increasingly complex, innovation is a sought-after commodity. The integration of disciplinary perspectives, knowledge and experience has been identified as a key ingredient in the development of innovative product solutions (Cooper, 1993; Paulus, 2000; Kelly, 2001). Carlile (2004) reveals, "Dorothy Leonard's (1995) statement that most innovation happens at the boundaries between disciplines or specializations tells us that working across boundaries is a key ingredient to competitive advantage, but also why innovation proves so difficult to create and maintain". Design development is changing as local and global design challenges become more complex and the integration of knowledge and experience from multiple disciplines, including both design and nondesign disciplines, is required to address these challenges.

In the last two decades, research in participatory design and interdisciplinary collaboration has been moving towards the use of generative tools to support nondesigners in the communication of knowledge, ideas and experience (Sanders, 2006). Knowledge may be viewed as both explicit, that which can be taught and learned, and tacit, based on subjective insight, personal experience and intuition (Nonaka \& Takuchi, 1995). A variety of approaches and tools have been developed to facilitate the externalization of tacit knowledge through two and three-dimensional visual representations (e.g. sketching, collaging, mock-up/prototypes). These visual representations provide a common language, supporting communication and the manipulation of ideas between team members with different disciplinary backgrounds 
(Carlile, 2002). A number of these tools specifically address collaboration at the front end of the design process, when team-members are new to each other and the design problem.

Prototypes are recognized as boundary objects (Carlile, 2002) that facilitate sociocultural relationships within collaborative teams (Chung, 2009, p. 143) and support communication and ideation at the early stages of design development. Cross-functionalcollaborative-prototyping (CFCPing) presented by Chung (2009) is one example of wellarticulated steps and principles designed to support interdisciplinary design development. CFCPing involves small teams of professionals with diverse disciplinary backgrounds working together to understand and develop design solutions using physical prototypes as a medium for discussion and discovery. Insights from early research using this method suggest that prototypes provide a fuller integration of disciplinary knowledge and expertise, helping designers conduct efficient collaboration more easily and thus creating concrete product concepts and definitions more effectively. However, this initial research was conducted in an academic setting and as part of graduate course work in interdisciplinary product design; it has experienced limited implementation with professionals in industry. Therefore, there is a need for more empirical research exploring the use if CFCPing in a wider variety of design projects and with more diverse experts (Chung, 2009, p. 145).

\section{An Opportunity for Empirical Research}

Certain characteristics of the Canadian healthcare system suggest this is one industry that may benefit from the use of CFCPing as a method supporting collaboration and design development. It is widely acknowledged that healthcare is a complex system, 
facing multi-faceted challenges that demand collaboration between experts from multiple disciplines. In addition, safety culture, employee involvement and inter-professional care are now recognized as important methods for improving patient safety (Curran, 2007; Health Force Ontario, 2007). Thus there is a need to identify methods that support interdisciplinary collaboration in the healthcare context.

Medication error is a well-recognized challenge that has received increasing attention since the Institute of Medicine report "To Err is Human" (Kohn et al., 2000). In 2004 the overall incidence rate of medical error in Canadian hospitals was reported to be $7.5 \%$ of annual hospital admissions, suggesting that about 185,000 were associated with adverse events and close to 70,000 of these were potentially preventable (Bakeret al., 2004). In the same year, the Canadian Institute for Health Information (CIHI, 2004) revealed that approximately $24 \%$ of identified adverse events were drug or fluid related. Pediatric medication error has received significantly less attention than medication error in the adult population. Evidence from the U.S. suggests that potentially harmful medication errors may be three times more common in pediatrics than in the adult population (Ghaleb et al., 2006).

In 2009, a Canadian pediatric hospital launched an interdisciplinary research project to identify factors associated with IV bolus morphine use in a pediatric surgical ward (Parush et al., 2010). Findings from this study suggested that two thirds of the identified risk factors were related to medication preparation, which is conducted in the medication room. The next step was to identify potential solutions to the identified risk factors. As medication preparation and delivery is the responsibility of multiple disciplines within the hospital, and the medication room presents a physical design 
problem, collaborative prototyping was identified as a tool that could be used to support interdisciplinary collaboration and concept development with representatives from nursing, pharmacy and design.

\section{Goals and Objectives}

The overarching goal of this research is to explore interdisciplinary interaction during early concept development and contribute to the development of a better understanding of how prototyping methods can be used to support collaboration in small groups. Therefore, it is necessary to study interactions between participants and visual representations during collaborative concept development. Cross-functional collaborative prototyping (CFCPing) has been identified as a method requiring further empirical study, and a pediatric hospital in need of innovative solutions for identified medication safety challenges provides an empirical context. This leads to the two main objectives of this thesis: to explore the use of CFCPing for interdisciplinary collaboration, and to investigate the use of collaborative prototyping to support communication between disciplines and early concept development in a healthcare context. Addressing these objectives helps to answer the following research questions:

1. What role(s) do professional product designers and prototypes play in the context of cross-functional collaborative prototyping at the front-end of design development?

2. How can CFCPing support interdisciplinary collaboration in a healthcare context? These research questions are contextualized by a review of relevant literature. 
INTERACTIONS IN COLLABORATIVE PROTOTYPING

\section{Literature Review}

\section{Concept Development at the Fuzzy-Front-End}

The early phase of the design process is described by some researchers as idea generation or concept development encompassing the process of identifying the main problems or design opportunities based on existing solutions and generating new ideas, improvements and design concepts (Boeddrich, 2004; Reinertsen, 1999; Vink, Imada \& Zink, 2008). It is an uncertain time in the product development cycle, also referred to as the fuzzy-front-end (Smith \& Reinertsen, 1991). In product design, this phase includes the period after customer needs are identified, when product concepts are developed, but before technical and financial limitations are introduced and a final concept selected (Dahan \& Hauser, 2002; Ulrich \& Epinger, 2000; Chung, 2009). One of the main objectives during this process is to generate ideas and explore solutions. During initial concept development "it is less about validating and filtering the ideas than it is about providing ongoing guidance in the uncertain innovation endeavor" (Fulton Suri, 2008, p. 56). The desired outcome of this exploratory period is an innovative product concept, defined as "the first abstraction of product ideas, which is a preliminary characterization of the future product" (Chung, 2010, p. 19).

The uncertainty and exploratory nature of initial concept development invites opportunities for innovation and chaos. The process can be lengthy, is often poorly understood and full of opportunities for improvement (Reinertsen, 1999, p. 25). Initial concept development is a critical point in new product development, setting the stage for later phases of product design and testing. Thus understanding how experienced and successful designers and design teams execute the concept development process, and how 
it can be improved, remains an important focus of study (Reinertsen, 1999; Cross, 2007). For example, protocol analysis of experienced designers working through the conceptual phase of a design problem has been used in an attempt to develop a better understanding of how good ideas are generated and developed (Schön, 1983; Cross, 2007).

The act of exploring the possible solutions and generating ideas, often referred to as brainstorming, is an important part of early concept development. Brainstorming in groups vs. alone is generally preferred for creative tasks and is a popular tool for generating new ideas in organizations (Sutton \& Hargadon, 2010). A significant amount of research has investigated factors contributing to more or less successful brainstorming and concept development. In particular, the integration of disciplinary perspectives, knowledge and experience within these groups has been identified as a key ingredient in the development of innovative product solutions (Cooper, 1993; Paulus, 2000; Kelly, 2001). However, interdisciplinary collaboration in itself also presents challenges. Thus it is important to identify factors affecting group brainstorming and define interdisciplinary collaboration in the context of design development before studying interdisciplinary collaboration before engaging in empirical study.

\section{Brainstorming and Creative Problem Solving in Small Groups}

A formal process for brainstorming was initially introduced by Osborn (1963) as a tool to support creative problem solving in groups. He introduced a number of strategies to improve the outcomes of group brainstorming in organizations, including: avoid criticism during idea generation, suggest wild ideas, combine and build on ideas, and the notion that more ideas produce more better ideas. These principles, or rules of brainstorming, have been widely adopted and hold particular value in the early concept 
development strategies used by a number of innovative design firms. One well-known example is IDEO, one of the largest design innovation and consulting firms in the United States, which shared some of its brainstorming and innovation strategies on $A B C$ Nightline in 1999.

Since Osborn's initial publication, a significant amount of research has been dedicated to investigating the effectiveness of the originally proposed brainstorming process and other factors that can influence the outcomes of small group work (e.g. Isaken, 1998; Turner, 1999). Measuring outcomes or group effectiveness is in itself a challenge. Quantity is one outcome measure that has been used in previous brainstorming studies (Paulus \& Brown, 2003), though counting ideas has also been identified as a challenge. Philp (2007) identified that a general idea is "broad or represents a theme but does not provide a concrete example of how this theme can or should be realized" whereas a specific idea is "a narrow representation of a broad idea". Despite this definition it is sometimes difficult to determine whether an idea is unique or not. In an attempt to address this challenge, the present research will count both general and specific ideas separately as well as the total.

Some of the factors that can be more or less controlled in the planning of group brainstorming sessions include: time, group size, selection of participants, presence of a facilitator, and scope of the problem to be addressed (Argote, Gruenfeld \& Naquin, 2001; Sutton \& Hargadon, 2010; Isaken, 1998). Many are dependent on the specific objectives of the study. For example the time allotted for brainstorming observed in literature varies from 15 to 30 minutes (e.g. Hymes \& Olson, 1992; Philp, 2007) and for collaborative concept development from 30 minutes to multiple days (e.g. Austin et al., 2001; Chung, 
2009; Dindler \& Iversen, 2007; Sutton \& Hargadon, 2010). All of these factors are addressed within the remaining literature review and description of the specific method selected for use in this study. The selection of participants starts to be addressed in the following section.

\section{Interdisciplinary Collaboration}

This research investigates 'interdisciplinary' collaboration. This term is not new and has been applied in a variety of situations including: interdisciplinary studies, interdisciplinary research, interdisciplinary care in hospitals, and interdisciplinary design development. Furthermore, this approach has been used in both research and practice in many areas including human-computer-interaction, marketing and business (Muller, 2003; Campbell et al., 2008). But because this term can have different meanings in different disciplines, it is necessary to clarify the meaning in the context of this research.

In general, the term 'interdisciplinary' is considered to mean the collaboration of multiple disciplines towards a common goal through competent communication (Turner, 2000, p. 52). This definition is further clarified by referencing the Oxford English Dictionary, which defines collaboration as the act of working in conjunction with others, co-operation and united labour. Synonyms include: to join forces, team up, band together, participate, combine, ally, pool resources, or put heads together. For the purpose of this study, 'interdisciplinary design development' implies a group of individuals from a diverse educational and/or professional backgrounds and experience (disciplines) working together (collaborating) to identify and conceptualize (develop) innovative products and/or services. In other words, varied disciplinary perspectives represented within a design team. 
The composition of an interdisciplinary design team is dependent on the disciplinary knowledge required to address the design challenge and perhaps the team leader's perspective on user involvement. The title of 'interdisciplinary team' has been used to describe teams of designers from multiple disciplines, for example: graphic, industrial, architecture or engineering, industrial, marketing (e.g. Carlile, 2004). It has also been used in the case of more diverse design teams that include non-designers (e.g. psychology, industrial, engineering, biology as in the teams that work at the renowned product design firm IDEO). In addition, interdisciplinary collaboration can also be used to describe teams that include end users and other stakeholders, as with participatory design methods.

\section{Participatory and User-Centered Design}

The traditional Scandinavian approach to participatory design, developed in the early 1970 s, calls for a cooperative approach to design development with professional designers and experienced users "acting as lay designers" (Bødker et al., 1988, p. 385). In this way, participatory design is similar to interdisciplinary design. However, in the majority of participatory design studies, designers or researchers are initiating and managing the process. In interdisciplinary initiatives, the designer may not have a predefined leadership role.

Participatory design evolved somewhat differently in North America and Europe, despite common Scandinavian roots (Gregory, 2003). In Europe, there appears to be more of a focus on the democratic origins of the method, which encourage user involvement in design development and decision-making. In these cases, participatory design practices may include end-users as well as other stakeholders who may be served 
or affected by the product. One example is Muller's (1992, p. 460) approach to software design that focused on "product quality (inclusion of expertise), product commitment, and workplace democracy".

In North America, participatory design practices evolved towards user-centered design, viewing users more as a subject of study. User-centered design (UCD) includes a variety of methods and tools for user research and design development that focus on developing solutions that meet the needs of identified end-users. Users can be more or less involved in various phases of the design process: from being the subjects of extensive study in the problem identification phase, explaining their view of current problems, to offering solution ideas and participating in testing and feedback on concepts presented by the design team. In many cases users are viewed as subjects to be studied by designers or design researchers and are not invited to be members of the design team. As stated by Brereton and Burr (2009, p. 102), "Participation in design is often sought in order to better understand 'users' so as to make more saleable commodities", which contrasts the traditional Scandinavian approach.

As the landscape of user-centered-design continues to evolve, non-designers are being invited to participate earlier and earlier in the design development process, not as subjects but as co-creators and members of the design team (Sanders, 2005). Thus there appears to be resurgence of the traditional participatory aspects of UCD in design research circles. For example, Sanders \& Stappers (2008) advocate co-design practices with collaboration between designers and non-designers, where non-designers include both researchers and users. Today, many design research consultancies bring together interdisciplinary teams of researchers and designers to develop in-depth knowledge and 
understanding of user needs in an effort to generate innovative product or system solutions (Sanders, 2008; Hoonhout, 2007; etc.).

After more than 30 years of participatory design practice, the evolution of UCD and the recognition that interdisciplinary collaboration is required to address complex problems that lie at the boundaries between disciplines there appear to be differing views on the involvement of users or stakeholders at the fuzzy-front-end. The following three examples are a case in point:

Participatory practices in ergonomics design. Vink et al. (2008) evaluated stakeholder involvement in participatory approaches to workplace and organizational improvements according to theory and practice, and highlighted stakeholder involvement in each step based on a review of projects of 300 practitioners. The generic steps in the design process were documented as follows: Introduction; Analysis; Idea Generation; Idea Selection; Prototyping; Testing; Adjusting; Implementation; Evaluation. For the purpose of this comparison, it is assumed that the 'fuzzy-front-end' would encompass the first three steps. Vink et al's evaluation shows that participatory ergonomics practices support the involvement of end users and subject matter experts as early as possible in the design development. Specifically, "In step 2 (analysis) employees should be involved because they understand the problems firsthand. In steps 3 (idea generation) and 4 (idea selection) employees should be involved because they may have ideas for improvement and they will work in the improved situation..." (p. 521). 
Philips design research experience. In his article for CoDesign, Hoonhout (2007) appears to differentiate between interdisciplinary design development at the front-end and co-design or participatory approaches at the front-end, excluding users from the interdisciplinary context. Experience at Phillips suggests that co-creation with consumers (end users) in the early creative phase of new product development does not seem feasible because the process of conceptualizing needs and uses for new or underdeveloped technologies may be too abstract for the average consumer to grasp (p. 22). Conversely, "bringing together domain experts from different backgrounds, creating a setting in which barriers between disciplines can disappear, and adopting a user-centric, rapid iterative investigation, ideation, prototyping and development process" is a promising approach (Hoonhout, 2007, p. 19). Thus it is suggested that experts from multiple disciplines use a "rapid iterative investigation, ideation, prototyping and development process" with no user-involvement at the outset. Hoonhout provides a series of examples from Phillips design where an interdisciplinary team of experts led product conceptualization. In these cases, consumers (end users) are not involved in the initial ideation process, but are brought in early to provide feedback/evaluate initial ideas according to a user-centered design approach.

The 'lead-user' perspective. Originally developed by Eric von Hippel (1988), this approach believes that innovation resides in the minds of expert users of existing products and services rather than manufacturers or designers. These 'lead users' are both motivated to innovate because they will benefit from the results, and experience 
needs earlier than others (Leonard, 2009). Thus user research conducted following von Hippel's approach targets and interacts exclusively with users at the leading edge of the target market.

The present research approaches interdisciplinary collaboration from a participatory perspective where end-users are involved as members of the design team from an early stage (Bødker et al., 1988; Muller, 1992; Gregory, 2003). From this perspective, design is viewed "as an open, innovative, user-involved, cross- disciplinary, and thus multi-perspective cooperative process" aimed at creating tools and systems that will improve the work life of end users (Broderson et al., 2008). The role of designers and non-designers in this type of activity is somewhat undefined and thus there is a need to know more about knowledge management and tools that support interdisciplinary collaboration in order to better define future roles.

\section{Knowledge Management in Interdisciplinary Collaboration}

Integrating disciplines poses challenges for communication and concept

development. Barriers between disciplines exist as a function of unique language, knowledge, and practice cultures rooted in professional training and experience. Particularly relevant to this research is the example presented by Hall (2005, p. 188), which describes professional cultures as barriers to interprofessional teamwork in healthcare and highlights that the "educational experiences and the socialization process that occur during the training of each health professional reinforce the common values, problem-solving approaches, and language/jargon of each profession”. Carlile (2002) describes the complexity of interdisciplinary collaboration in organizations in terms of 
three perspectives of boundaries: syntactic, semantic and pragmatic. Crossing syntactic boundaries requires a common language or syntax for analysis that will enable knowledge transfer between collaborators. Semantic boundaries require common understanding and a mechanism for knowledge translation or interpretation. Pragmatic boundaries refer to practical barriers between players and the value associated with acquired knowledge. A political approach and requirements for knowledge transformation or developing new beliefs, understanding, and perspectives that were not previously possessed by any of the players involved is discussed in the context of political barriers to collaboration. This last boundary type is comparable to the cultural barrier articulated by Hall (2005).

Carlile (2004) presents a framework for managing knowledge across syntactic, semantic and pragmatic boundaries between disciplines through processes of knowledge transfer, translation and transformation. For example, members of an interdisciplinary team might be able to overcome syntactic boundaries if they speak the same language (e.g. English). However, in order to transfer knowledge team members must have the same understanding of the words being used. For example, in the author's own interdisciplinary team experience the word 'scenario' was revealed as having a different meaning in different disciplines. In one design project, members of the team with an information technology systems or architecture background identified scenarios as an illustrated snapshot in time. To others with industrial design and psychology backgrounds, a 'scenario' illustrated, either graphically and/or in words, the conduct of an activity in time and space. This led to some confusion, which was resolved upon viewing the 'scenario' in question presented as computer-rendered snapshots of people in 
a hospital room, the design of which was the object of the meeting.

Verbal or written language may be insufficient to cross semantic boundaries, especially when the knowledge to be transferred cannot be expressed in this medium. For example, 'tacit' knowledge, defined by Nonaka \& Takeuchi (1995, p. 8) as something not easily expressible, highly personal and hard to formalize, "is deeply rooted in an individual's action and experience, as well as the ideals, values, or emotions he or she embraces". Tacit knowledge can sometimes manifest as insight, intuition, or a hunch, the reasoning behind which is hard to explain. To support collaboration between disciplines it is important that each team member be able to communicate and explain their knowledge to others so that it can be evaluated for its contribution to solving the problem. Using verbal language as the only means to cross the boundaries between disciplines can lead to misunderstandings between interdisciplinary team members. Thus there is a need for other mechanisms or tools that support the transfer, translation and transformation of knowledge.

\section{Boundary Objects}

Boundary objects are physical artifacts that can be used to support knowledge management between disciplines. This concept was originally defined by Star \& Greisemer (1989) as artifacts that occupy multiple intersecting worlds and satisfy information requirements in each of them. In product design and knowledge management circles these are physical objects that support the transfer, translation and transformation of knowledge between collaborating disciplines (Carlile, 2002). Examples of boundary objects relevant to this study include sketches and prototypes, which are visual representations of information and ideas. 


\section{Visual Representations}

Visual representations are a form of external representation of thought and thought process. People use external representations, i.e. material and physically perceivable representations, to share information and communicate thoughts and ideas, both with others, and as part of their own problem solving process (Goldschmidt, 2004). One of the reasons external representations are so useful, is that they support the cognitive process by "serving as a cognitive tool to augment memory and information processing by relieving the mind of some of those burdens" (Tversky, 2002, p. 1). This is part of the reason why people write and draw when problem solving. Essentially external representations function as a sort of external memory. Recording this information or knowledge 'in the world' frees the mind to devote energy to exploring other ideas without having to keep track of pre-identified information and ideas. Though a certain amount of information can be stored in working memory, external representations of thought support the extraction of a much larger repertoire of information and serve as predicates to new ideas (Chandrasekaran, 1999). It is conceivable that these advantages hold for both individual and group problem solving situations.

It is often said that 'a picture is worth a thousand words'. This is supported by research that indicates "expressing ideas in a visuospatial medium makes comprehension and inference easier than in...language" (Tversky, 2002, p. 1), where visuospatial refers to the perception of spatial relationships among objects. As product design is primarily a spatial domain, it is no surprise that visual representations are heavily used. In fact it seems that visual representations are the "lifeblood of the activity of design - not only are diagrams, sketches, and CAD drawings used during design in domains that are 
intrinsically spatial, but they are also used extensively in design domains that are not intrinsically spatial, such as software design and planning" (Chandrasekaran, 1999, p. 1).

Types of visual representation include a variety of imaging methods such as rough and detailed drawings and diagrams, physical mock-ups or prototypes, animation and video - all for different purposes at different stages of the design process. Each of these methods offers different affordances, benefits and limitations depending on the object or system being designed or the problem being solved as well as the stage of the design process at which they are being implemented.

A variety tools that allow for two, three and four-dimensional visual representation have emerged in an effort to develop a common language between disciplines and harness the knowledge and experience of users at the fuzzy-front-end of the design development process. Many of these tools have evolved from user-centered and participatory design approaches (Bodker, 1988; Muller, 1998; Muller, 2002; Sanders \& Chan, 2007) and incorporate methods that facilitate visualization of future activities and solutions, including sketching, mock-up/prototyping, collaging, video creation (Broderson, Dindler, \& Iverson, 2001; Kelly, 2008; Sanders, 2005; Sanders \& Stappers, 2008). For example, Muller (1992, p. 455) "uses low-tech, paper and pencil approaches in order to facilitate the direct participation in software design by users who may not be computer-literate" and "video recording for record-keeping, and to simplify dynamics of the design session". Sharp, Rogers \& Preece (2007) present a number of case studies where focus groups have been tailored to emphasize collaboration and facilitation between stakeholders and researchers. Two of these examples involve soliciting ideas from users and asking them to make models of their favorite ideas, similar to Sanders 
(2006). A third involves workshops where users and developers work together to develop product requirements (Wood \& Silver, 1995). This kind of approach as is sometimes referred to as 'collaborative prototyping' (Chung, 2009) or 'co-design' (Muller, 1992; Sanders \& Stappers, 2008).

According to the New Oxford American Dictionary (2005), the term 'prototype' can be used to describe a first example, model, mock-up, or sample. "In the traditional sense, we may consider prototyping a collaborative identification of possible futures, rooted in current practice but with the purpose of introducing change" (Broderson et al., 2007 , p. 20). In other words, prototypes are representations of design concepts that are used to test these concepts. Floyd (1984) distinguishes between prototyping for exploration, experimentation and evolution based on the type of design challenge faced by the team. Prototyping for exploration is defined as "where the emphasis is on clarifying requirements and desirable features of the target system (or product) and where alternative possibilities for solutions are discussed" (Floyd, 1984, p. 6). Fulton Suri (2008, p. 56) identifies early prototypes as a "visible or tangible representation of an idea, to be thought of as a probe or thought-experiment; it is not a full-fledged pilot or a preproduction version of the real thing". Early prototypes are often rough mock-ups made with available or found materials, developed during initial concept development, as opposed to more refined prototypes that might be used for testing developed concepts (e.g. three dimensional CAD models).

It is acknowledged that prototypes allow for physical representation of conceptual ideas (Logan \& Radcliffe, 2001) and function as mediating artifacts or boundary objects (Carlile, 2002) that can be used to support translation, transfer and transformation of 
knowledge between disciplines (Carlile, 2004). Chung (2009, p. 8-9) applied this theory to cross-functional (interdisciplinary) design development projects and observed "that prototyping in collaboration tends to promote mutual learning between individuals with different types of expertise and that prototypes play the role of 'scaffold' by increasing the efficiency of the learning experience". Furthermore, "prototypes play a critical role in making uncertain situations more certain" (Chung, 2009, p. 44). Cross-functionalcollaborative-prototyping (CFCPing) presented by Chung (2009) is one example of a well-articulated method designed to support design development involving a diversity of disciplinary expertise in the spirit of co-design. Insights from early research using this method suggest that prototypes provide a fuller integration of disciplinary knowledge and expertise, helping designers conduct efficient collaboration more easily and thus creating concrete product concepts and definitions more effectively. However, this research has been conducted largely in academic settings, and as part of graduate course work in interdisciplinary product design, and has experienced limited implementation with professionals in industry. Therefore, there is a need for more empirical research exploring the use if CFCPing in a wider variety of design projects and with more diverse experts (Chung, 2009, p. 145). As this is one of the motivations for the present study, the CFCPing method and principles are presented in detail.

\section{Cross-Functional Collaborative Prototyping (CFCPing)}

CFCPing involves a team of experts with diverse disciplinary backgrounds working together to understand a design problem using physical prototypes as a medium for discussion and discovery. It offers a means to facilitate interdisciplinary team communication and idea generation (Chung, 2008). The method is based on three modes 
of action and guided by seven principles defined in Chung's doctoral dissertation, published in 2009. The three modes of action are presented first, followed by a description of the method and seven principles.

\section{Modes of action.}

The three modes of action required for effective cross-functional collaborative prototyping are: ideation, embodiment and critique. These modes emerged from Chung's theoretical analysis of collaborative prototyping for early concept development, which was framed by activity theory. Each of these modes has a role in supporting collaborative concept development and knowledge management between disciplines, as follows:

1. Ideation is defined as the process of sharing domain-specific information and generating ideas. It includes both identifying and understanding the design problem and is "more productive when people physically interact with a tangible artifact, which helps to share different perspectives for discussion" (Chung, 2009, p. 103).

2. Embodiment is the act of making ideas tangible. It is not only about visual representation, or fabrication of a pre-conceived notion, but also the potential to test and develop new ideas, as discussed in the previous section. Embodiment is about developing shared knowledge within a group.

3. Critique is described as a process of analyzing limitations, problems and conflicts that allow participants "to refine suggested ideas and narrow down to specific concepts" (Chung, 2009, p. 107). It is about testing the embodied concepts, and developing new knowledge. 
Chung (2009, p. 108) emphasizes "iterations of the three modes of action are critical for turning an initial idea into more appropriate and clearer product concepts". The desired outcome, where "initially abstract and vague ideas materialize through repeated embodiment and are improved through critique" (Chung, 2009, p. 118) is clearly reflected in the proposed CFCPing method.

\section{The CFCPing method.}

Before the session, organizers should do the following:

1. Select participants with knowledge and experience relevant to the design problem (p. 110).

2. Prepare reference and prototyping materials that support "support rapid representation of design ideas" (p. 110). Paper, duct tape and modeling clay are used as examples.

3. Ensure there is enough space and work surface(s) to allow participants to collect as a whole group and break out into smaller groups for focused discussion and prototyping.

The session should start with an introduction by the organizer providing background information on the design problem, specific objectives and the agenda for the session. The three modes of action: ideation, embodiment and critique, should be explained and emphasized as important acts expected of all participants throughout the session (Chung, 2009, p. 12). Then participants should have an opportunity to share their background and experience before being divided into small interdisciplinary teams of between three and seven participants. The remainder of the session is comprised of a series of small group breakouts: preliminary ideation (45 min), preliminary prototyping 
(45 $\mathrm{min}$ ) and final prototyping ( $90-120 \mathrm{~min}$ ), each of which is followed by a 10 -minute presentation of ideas and discussion with the larger group. At the end of the session is a concluding discussion, identifying the accomplishments, challenges and future directions as a result of the activity. A detailed schematic of the process is presented in Figure 1, which has been used with permission. According to information presented this figure, the total duration of the session is approximately five hours. 


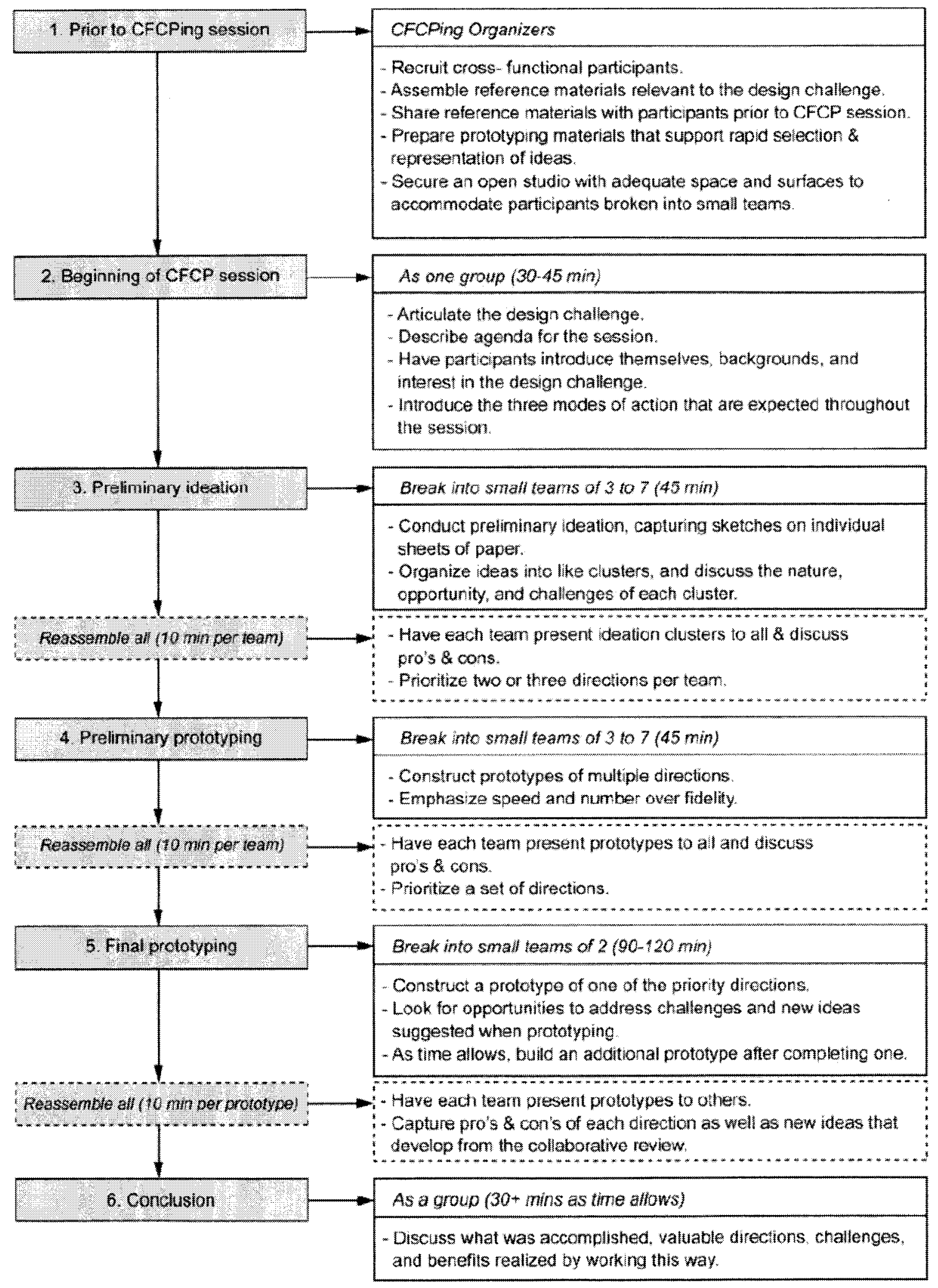

Figure 1. CFCPing Method by WonJoon Chung (2009, p. 116). Used with permission. 
The seven guiding principles for conducting effective sessions represent one of the most important aspects of the CFCPing method. These seven principles are based on Chung's review of supporting literature and his own research using early collaborative prototyping:

1. Provide a description of the design goal and access to related reference materials.

2. Facilitate iteration of the three modes of actions during the entire CFCPing process.

3. Recognize that finding a failure is as beneficial as finding a solution idea: mistakes can often lead to important learning opportunities

4. Identify and explore misinterpretations through discussion and prototypes. One of the challenges of interdisciplinary collaboration is establishing shared understanding. Investigating the nature of miscommunications and misunderstandings can lead to mutual learning.

5. Favor manipulation over fabrication.

6. Mutual learning is the engine for forward momentum in team collaboration.

7. Iterations, incorporating changes in response to learning, improve the quality of an outcome.

Regarding the fifth principle, Chung states that non-designers hesitated to execute their ideas, but seemed happy to modify pre-existing models or artifacts, and suggests two ways to tackle this problem: "by providing flexible materials that are easy to manipulate and by having someone skilled make prototypes on call to provide more advanced fabrication quickly" (p. 121). 
As previously mentioned, insights from early research using CFCPing suggest that the prototyping method described here helps to provide a fuller integration of disciplinary knowledge and expertise, leading to more efficient collaboration and more product concepts and definitions. Limited implementation of this method with professionals in industry suggests there is a need for more empirical research exploring the use if CFCPing in a wider variety of design projects and with more diverse experts (Chung, 2009, p. 145). After recognizing this need, a suitable research context needed to be identified. The healthcare industry was identified as a suitable focus based on personal interest and the fact that it is a complex environment, faced with numerous product design and integration challenges, and requiring a high degree of interdisciplinary interaction.

\section{The IV Morphine Study}

In 2009, a Canadian pediatric hospital conducted a prospective study to identify factors contributing to medication safety. The study was designed to identify top risk contributors and generate product and system-based solutions to address identified opportunities for improvement based on a focused study of IV bolus morphine use in a surgical ward. The ultimate objective was to improve medication safety and nursing work life in the hospital.

An interdisciplinary team including representation from pharmacy, psychology, nursing research and practice and design research was responsible for the planning and execution of this study. The study involved: ethnographic observations and interviews with surgical unit staff to identify risk factors, subjective severity ranking with experienced nursing staff to support prioritization of the identified risk factors, and focus 
groups do develop recommendations addressing top risk contributors. The author of this thesis was intimately involved with all phases of the IV morphine study.

Initial results from the observation portion of the study are presented in Parush et al. (2010). When combined with findings from the interviews and severity ranking, analysis showed a significant number of identified risk factors were related to drug preparation and medication room design (Campbell et al., 2010a). The medication room is the secure area where narcotics are stored and prepared by nursing staff preadministration. There was a general consensus amongst staff, management and the study team, that a re-design of the medication room and/or medication management system could make a significant contribution to safe IV morphine use at the hospital. These findings are supported by literature that highlights pediatric medication errors and recommended practices not currently implemented at the hospital, including: unit dose systems, electronic documentation and automated medication dispensing cabinets (e.g. ISMP, 2008; Sharek et al., 2008).

At this point the research team was clearly situated in the fuzzy-front-end of design development, with a good idea of the problems and drivers for changing current medication management practices and tools but, without a clear picture of an appropriate solution for the pediatric surgical ward.

A series of collaborative concept development sessions were proposed as a means to bring together experienced professionals representing the disciplines of nursing, pharmacy and industrial design to develop solution concepts through interdisciplinary collaboration. Cross-functional collaborative prototyping (Chung, 2009) was identified as a tool that could support concept development and knowledge management between 
disciplines. Thus the pediatric hospital context and medication safety was identified as the context within which to investigate interdisciplinary interactions and the use of prototyping for early concept development. 
INTERACTIONS IN COLLABORATIVE PROTOTYPING

\section{Methods}

\section{Approach}

The overall research strategy was exploratory. Cross-functional collaborative prototyping (CFCPing) sessions were conducted in an effort to observe interactions between disciplines and specifically the role played by industrial designers and prototyping in collaborative concept development at the fuzzy-front-end of the design process. The goal of using this approach was to gain deeper understanding of the collaborative process through observation of interdisciplinary teams at work. The collaborative sessions were conducted in the context of a larger prospective study designed to identify factors contributing to IV bolus morphine safety and develop product and system-based solutions to address top risk contributors. A protocol for the collaborative sessions was developed and piloted prior to conducting the focused study with hospital staff and professional designers.

As this thesis is interested in the interactive behaviour of interdisciplinary participants with each other and with prototypes, systematic observation was selected as the primary method of inquiry (Bakeman \& Gottman, 1997). Observational data was collected by video recording the collaborative sessions. This was used to identify verbal and physical interactions between participants, with reference material and with prototyping materials. Feedback questionnaires were distributed at the end of each session and used to evaluate participant's perception of their role and the role of other team members in the collaborative activity, and the use of prototypes to develop solution concepts. 
A schematic of the research activities is presented in Figure 2, and the remainder of this section describes each activity in detail.

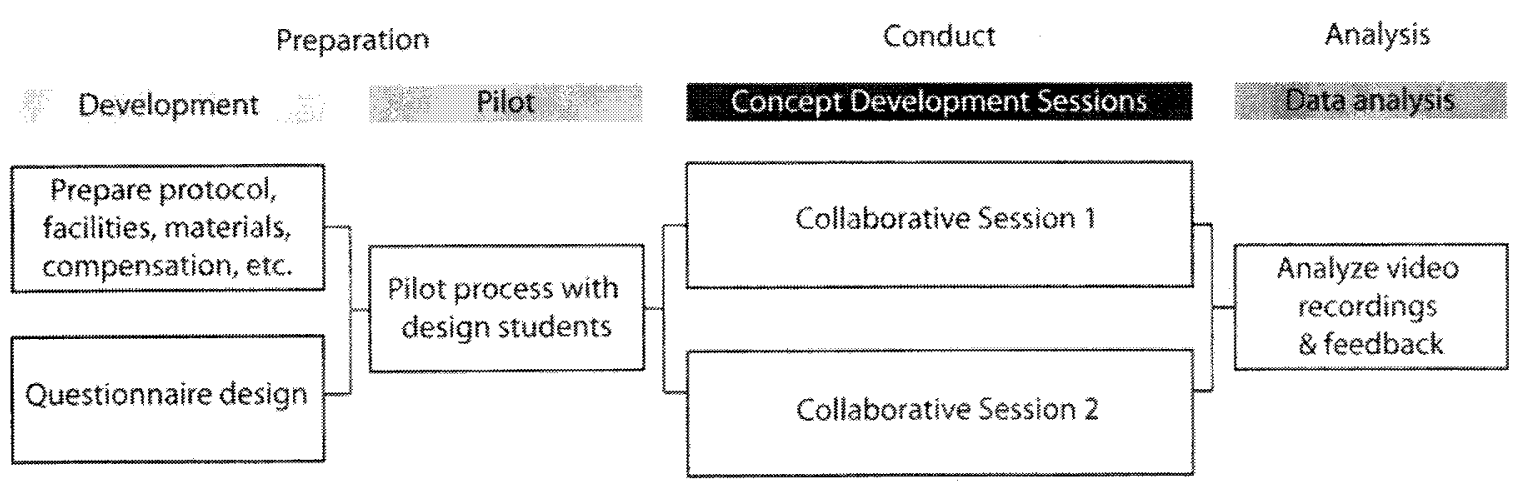

Figure 2. Research activities

\section{Protocol Development}

The protocol was developed in the context of literature on brainstorming and group creativity (Paulus \& Nijstad, 2003; Mathieu, Maynard, Rapp \& Gilson, 2008; Argote, Gruenfeld \& Naquin, 2001), co-design (Hoonhout, 2007; Sanders \& Stappers, 2008; Vink, Imada \& Zink, 2008; Westerlund, 2007), and collaborative prototyping (Chung, 2009; Muller, 1992; Bodker \& Grønbœk, 1996). The following variables were identified and considered during protocol development: demographics of the group (participating disciplines, experience of participants, number of participants), duration, location and materials, description of the problem/challenge provided to participants, and the number of small groups observed.

The general structure of the collaborative sessions was modeled after Chung's (2009) Cross-Functional Collaborative Prototyping (CFCPing). When applying this method in the pediatric hospital context, modifications were required in the interest of feasibility and acceptance by participating disciplines. The most significant variation was shortening the overall duration of the exercise by opting for two cycles of small group 
work followed by large group discussion, rather than the recommended three cycles. The motivation for this decision was the perceived time that volunteers could be expected to accept, according to feedback from management and research staff at the hospital. The author was fortunate enough to consult with the original author of the CFCPing method during the development of the protocol.

The structure of the collaborative sessions conducted in this study was as follows: (1) Introduction by lead facilitator, (2) Breakout 1: divide into small groups \& brainstorm solution concepts, (3) Sharing of ideas with the larger group, (4) Breakout 2: use collaborative prototyping to develop solution concepts, (5) Presentation of solution concepts to the larger group and discussion. This process is presented in Figure 3, wherein each small group is represented by a group of three, representing the three disciplines that participated in this study: industrial design, nursing, pharmacy.

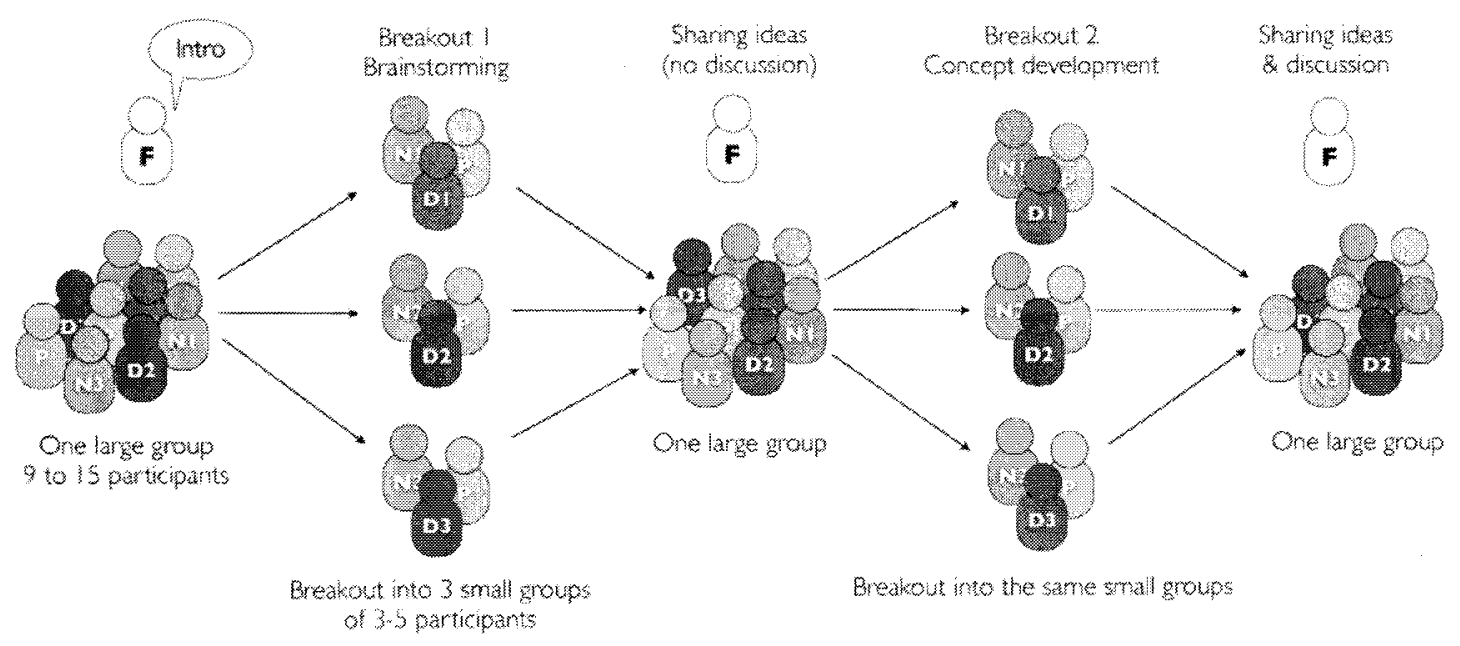

Actors

$N=$ Nurse

$\mathrm{D}=$ Designer

P- Pharmacistphamacy ectmician

$F=$ Lead falltator

Figure 3. Collaborative session structure 
The following three sub-sections present key elements of the protocol that was developed and approved by the research ethics board at the hospital. A copy of the approved protocol is presented in Appendix 1, including the informed consent form for participants.

\section{Participants.}

The study was designed to include hospital staff and industry professionals with expert knowledge related to the identified design problem, including: pharmacists, pharmacy technicians, surgical ward nursing staff, nursing management, and industrial designers. The following inclusion criteria were based on the minimum knowledge deemed necessary to support the development of solution concepts for narcotic safety management on the surgical unit:

1. Participants employed by the hospital were required to be either currently working on the surgical unit, have a professional interest in pain management or patient safety and be knowledgeable about IV bolus morphine delivery, the pharmaceutical characteristics of the drug, and/or some aspects of the existing preparation and delivery process in the context of pediatric care.

2. Designers recruited to participate in the study were professional designers with a minimum of five years of work experience. A combination of post-graduate work and practical experience was deemed acceptable.

\section{Duration.}

The CFCPing schedule was shortened from a five-hour workshop (as presented by Chung, 2009) to a two and a half hour workshop by eliminating the third group 
breakout and shortening the time allowed for each of the remaining two breakouts. The length of brainstorming sessions and collaborative sessions observed in literature supports the shortened development time allocated for Breakout 1 and 2.

\section{Location and materials.}

The session required meeting rooms at with enough space to accommodate 12 participants together and also space to break into four small teams. These rooms needed to be outside normal work environments for all participants, so that participants from different disciplines would be on equal ground. Research and management at the hospital felt that it would be very difficult to get participants if the study was not conducted at the hospital. Therefore meeting rooms were located in a separate area of the hospital.

The following materials were provided to each group:

1. Reference images of the current surgical unit medication preparation equipment, tools and room layout.

2. Reference images of existing products, not currently in use at the hospital, as inspiration, and to increase awareness of the technology currently available.

3. Low-tech prototyping materials, including paper, markers, post-it notes, Styrofoam, modeling clay, tapes, etc. that would be easy to manipulate, shortening the time required for construction (Chung, 2009, p. 111), and also not intimidating, so as to facilitate the direct participation of non-designers (Muller, 1992, p. 455) who may not be familiar with prototyping materials and practices. Four digital video cameras with table microphones were used to record the session and capture audio and video of each small group for future analysis. 


\section{Introduction to the problem.}

The Introduction, provided in presentation format by the lead facilitator, was designed to introduce participants to the problem: medication error, IV bolus morphine administration in the pediatric surgical unit, and the identified top risk contributors, present the collaborative design process, the objectives of the session (to develop solution concepts addressing the identified top risk contributors), and the low-tech prototyping materials available to support concept development. Presentation slides with images and text were prepared to reflect this content.

\section{Feedback Questionnaire}

As previously mentioned, a feedback questionnaire was distributed to all participants at the end of each collaborative session. The purpose of the questionnaire is to evaluate participant's perception of (1) their role and the role of other team members in the collaborative activity and (2) the use of prototypes to develop solution concepts. Of particular interest were any differences between disciplines. Following is a brief overview of the questionnaire design. A copy of the final questionnaire that was distributed to study participants is presented in Appendix 2.

The questionnaire was divided into four sections: (1) Demographics, associating each respondent with a specific discipline, (2) Methods, asking participants their perception of the structure, materials provided and activities conducted during the collaborative session, (3) Group dynamics, including questions regarding the organization and conduct of small group activities, and (4) General impressions, asking participants their perception of the exercise as a whole. Questions related to methods and general impressions were scored on a Likert scale of one through five, where one was 
always very negative and five very positive. The group dynamics section consisted of multiple-choice questions. Only three questions in the questionnaire provided space for further explanation or comments from respondents.

\section{Preliminary Studies}

Two pilot studies were conducted to help prepare the researcher in delivering clear and concise instructions to participants, test video recording equipment and test the appropriateness of the reference materials and simple prototyping materials provided to each group. These full-length pilot sessions were conducted in the Master of Design Studio space at Carleton University. Master of Design students from Carleton University and nursing students from the University of Ottawa were invited to participate in the pilot studies in an effort to simulate the range of disciplinary backgrounds that would be present in the hospital study. Participation was voluntary and token compensation provided.

Unfortunately no nursing students were able to attend either session, which limited the extent to which the interaction between disciplines could be observed in the pilot study. However, a nurse researcher participated in the second pilot, bringing relevant domain knowledge and an interdisciplinary perspective to group activity. This participant also provided some insight into how hospital staff might respond to the collaborative method and prototyping activity.

Three Master of Design students participated in the first pilot session. This allowed for testing of the introductory presentation, general schedule of activities, appropriateness of materials and lead facilitator's own time management. It did not allow for testing of the complete schedule and sharing of ideas between small groups. As there 
was only one group, a single, longer, concept development session was used with a final presentation of mock-ups and summary of ideas. Participants completed draft feedback questionnaires and entered into a discussion on the format of the collaborative session and possible improvements to the protocol and questionnaire.

Some revisions to the introductory presentation were made based on feedback and lessons learned from the pilot study. The initial approach for the introduction of collaborative prototyping and interdisciplinary design development was for the facilitator to present an animated verbal account of the general concept and benefits accompanied by a set of slides with photographs of previous studies and industry experience. Feedback from the first pilot session led to the use of a clip from the IDEO Deep Dive (ABC News, 1999) as a means to introduce the collaborative prototyping method and interdisciplinary collaboration for design development to participants. A six-minute clip was prepared for testing in the second pilot, including the introduction of interdisciplinary team collaboration, user-involvement, brainstorming methods, and the utility of prototyping and time pressures. The second revision was the addition of a warm-up game of Pictionary, where non-designers were assigned as 'artists' for each team. This was intended, not only to loosen up the group and encourage a social, fun atmosphere, but also to demonstrate that less refined visualization skills and low fidelity representations were more than sufficient to communicate ideas to other team members, and to encourage non-designers to use visual communication and prototyping during small group collaboration.

In the second pilot session two small groups, consisting largely of master students with a variety of design backgrounds, completed the full two and a half hour 
collaborative concept development activity, including a short warm-up game of

Pictionary, and two breakout sessions followed by presentations by each team of the

concepts and prototypes developed. Again, participants completed draft feedback

questionnaires and discussed the format of the collaborative session and possible

improvements to the protocol and questionnaire.

The video approach not only shortened the presentation, but was also perceived to be more convincing by participants. The revised introductory presentation is presented in Appendix 3.

\section{Procedure}

The cross-functional collaborative prototyping schedule used to conduct the focused study is presented in Table 1 . This is the procedure that guided the focused study conducted as part of this research.

Table 1.

Collaborative session schedule

\begin{tabular}{llll}
\hline Time & Activity & Actors \\
\hline $10 \mathrm{~min}$ & $\begin{array}{l}\text { Introduction: Facilitator presents the design challenge, objectives, method } \\
\text { and schedule. Participants are divided into interdisciplinary teams. }\end{array}$ & Lead facilitator \\
\hline $5 \mathrm{~min}$ & $\begin{array}{l}\text { Warm up! A short Pictionary game designed to get non-designers All } \\
\text { comfortable drawing and making. }\end{array}$ & Participants \\
\hline $15-30 \mathrm{~min}$ & $\begin{array}{l}\text { Breakout 1: Brainstorming and preliminary concept development } \\
\text { Spend } 30 \text { minutes discussing and sketching ideas on how to reduce } \\
\text { interruptions and distractions through design }\end{array}$ & Participants \\
\hline $10 \mathrm{~min}$ & Brief Presentation of ideas to the large group (no discussion) & Participants \\
\hline $45-60 \mathrm{~min}$ & $\begin{array}{l}\text { Breakout 2: Concept development for top risk contributors (Break/snack- } \\
\text { time included) } \\
\text { Spend } 45 \text { - 60 minutes developing ideas related to the top risk } \\
\text { contributors (see handout). Use the materials provided to } \\
\text { build/make tangible representations of your ideas }\end{array}$ & Participants \\
\hline $15 \mathrm{~min}$ & $\begin{array}{l}\text { Final presentation of concepts using early prototypes as a basis for } \\
\text { demonstration \& discussion }\end{array}$ & All \\
\hline $10 \mathrm{~min}$ & Debrief \& request feedback from participants (distribute questionnaires) & Lead facilitator \\
\hline $5 \mathrm{~min}$ & Thank you \& distribution of compensation packages &
\end{tabular}




\section{Focused Study at the Hospital}

Representatives from the disciplines of pharmacy, nursing and design were invited to participate in the collaborative concept development sessions by email and announcements at staff meetings. The researcher visited the surgical unit and pharmacy to answer any questions staff might have in advance of agreeing to participate professional designers were contacted by phone and given the opportunity to ask questions in advance of the session. All participants were provided with informed consent forms in advance of the sessions.

Two collaborative sessions were conducted following the collaborative concept development session protocol and schedule described in previous sections of this thesis. The sessions were conducted from $3: 30 \mathrm{pm}$ to $6: 30 \mathrm{pm}$ on two separate days. Participants were involved in one session each, not both.

Both sessions were conducted in two meeting rooms at the hospital. These rooms were outside normal work environments for all participants. Both rooms had a number of small tables, for teams to work at. One room was larger than the other, with adequate space and seating to facilitate presentation and discussion of ideas and prototypes by the larger group.

Each small group was provided with reference images of the current surgical unit medication preparation equipment, tools and room layout, a handout of the existing floor plan of the medication room, surgical unit and pictures depicting the current tools and context as well as a selection of available medication management and dispensing technologies (not currently used at the hospital). In the second collaborative session, participants were only provided with the handout pictures and no floor plan of the 
existing context. This was done because during the first session participants spent a lot of time interacting with this reference and all three resulting design concepts fell within the rectangular shape of the existing floor plan. It was hypothesized that by removing this resource, the teams might be better able to 'think outside the box' and consider alternative medication management solutions.

Four digital video cameras with table microphones were set up to record the session. One camera and microphone was dedicated to each group. The fourth camera was used to record the introduction and large group interactions, and intended as a backup should any of the other three cameras fail. None did, and this camera provided an additional camera angle for one of the small groups.

The researcher, who was the lead facilitator for the session, delivered the introductory presentation. In both sessions, the introduction took longer than the scheduled 10 minutes because of question and answer by participants. In the first collaborative session this led the researcher to skip the warm-up game and move straight into group work in the interest of time-management. Small changes were made to the introductory presentation delivered in the second collaborative session in an effort to continuously improve the description of design objectives and CFCPing process that was provided to participants. A Pictionary warm-up game was conducted in the second session.

After the introduction participants had an opportunity to ask questions about the problem and process described before being divided into three small interdisciplinary groups. The groups were given 20 minutes for initial brainstorming (Breakout 1) then asked to present their ideas to the other teams before continuing through a 45 -minute 
concept development and prototyping period (Breakout 2). Integrated break time (refreshments were available throughout the session) meant that each group had an average of 38 minutes of concept development and prototyping in Breakout 2. Finally, all groups were asked to present their final concepts and a short discussion followed.

Immediately following each session, participants were asked to complete the anonymous feedback questionnaire providing their perception of the workshop, collaboration and the use of visual representations within their group.

\section{Description of the Data Sample}

The two collaborative sessions conducted as part of this study included six small groups, each with two to five participants $(\mathrm{M}=3.6)$ for a combined total of 22 participants across three disciplines. For identification purposes, each group was assigned a unique alphanumeric identifier according to the collaborative session (CS) and group number (G), e.g. the second group in the first collaborative session is labeled as CS1G2. The breakdown of participants from each discipline in each group is presented in Figure 4.

Representation from each of the three disciplines varies across the six groups, which precludes direct comparison between groups. However a qualitative comparison is possible, across all groups excluding CS1G2, which has only two members and is missing representation from pharmacy.

Some participants were unable to participate for the entire session. Specifically, one nurse had to leave approximately 10 minutes into Breakout 2, a pharmacist left 10 minutes before the end of Breakout 2 and the nurse manager was only present for the introduction and 10 minutes into Breakout 1. 


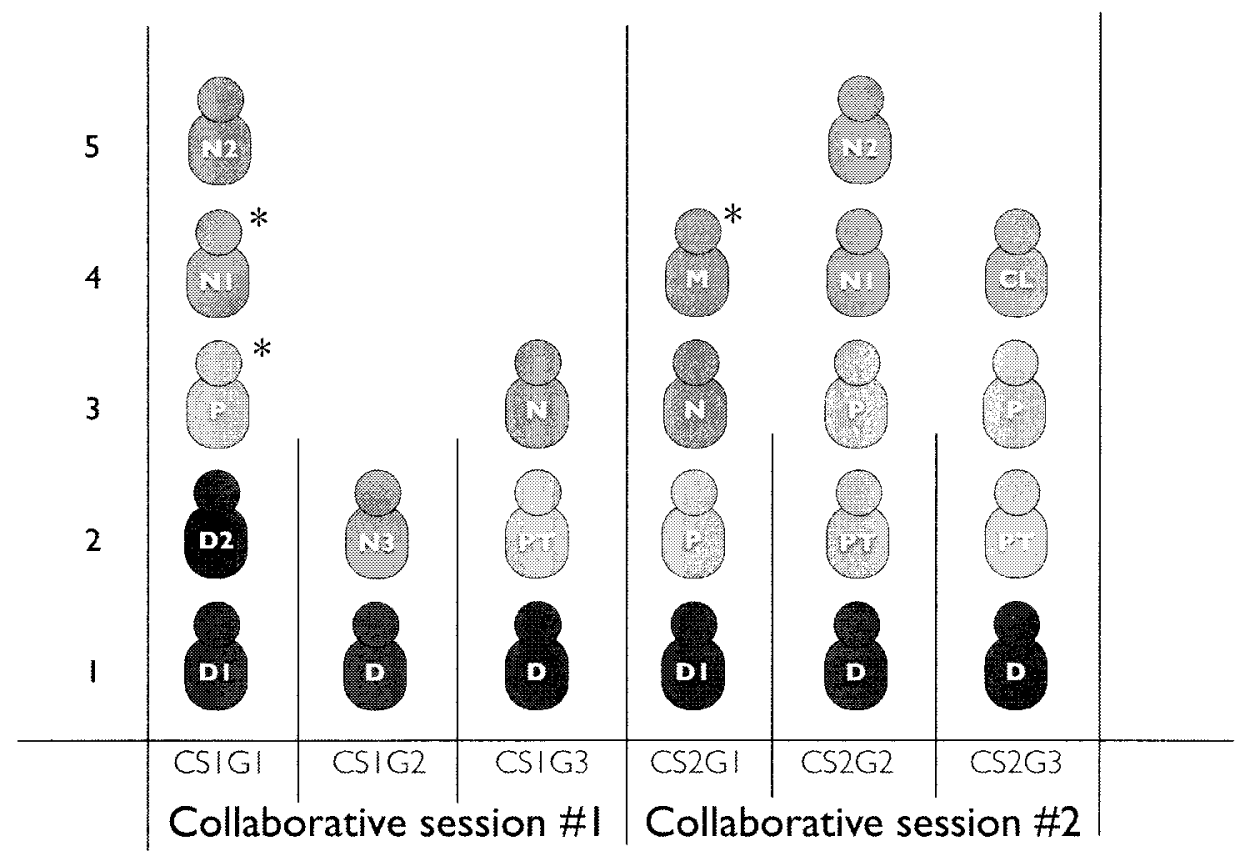
Participants Nursing $N=$ Nurse, $M=$ Nurse Manager, $C L=$ Clinical Leader
Pharmacy $P=$ Pharmacist, $P T=$ Pharmacy Technician
Design $\quad D=$ Designer

* Indicates participants that had to leave early

Figure 4. Breakdown of participants in each group by discipline

\section{Video Data}

During each session, one video camera was focused on each small group during the sessions to capture audio and video data of small group activity. As a somewhat adhoc process requiring group coordination by the lead facilitator the exact length of time allowed for each activity varies slightly between groups. Based on the captured video data, the mean duration of Breakout 1 is 23 minutes (SD 3.7) and Breakout 2 is 37 minutes and 42 seconds (SD 0.52).

After the session, it was discovered that the video recording for CS2G3 had poor quality audio, which means that analysis of verbal interactions was not possible for this group. However, qualitative analysis of physical behaviours was still possible. In 
addition, due to a delay in changing the videotape for CS2G1, 15 minutes of group activity at the beginning of Breakout 2 was not recorded. The remaining 25 minutes of group activity for CS2G1 was subject to qualitative analysis. Therefore, the full data set used in this study includes video recording of five small groups during Breakout 1 and three small groups during Breakout 2. This includes observation of a total of 6 designers and 13 non-designers, including nursing and pharmacy staff and represents approximately 2128 verbal utterances across Breakout 1 and Breakout 2 for the groups that were analyzed.

\section{Questionnaire data.}

Of the 22 participants in this study, 19 completed and returned the feedback survey distributed at the end of each collaborative session. One designer and two nursing responses are missing from the questionnaire data, and one nursing respondent did not complete the second page of the questionnaire, missing questions 10 through 16 out of 19. Thus the overall response rate was $84 \%$, distributed across all disciplines. 


\section{INTERACTIONS IN COLLABORATIVE PROTOTYPING}

\section{Analysis}

Qualitative data analysis, including systematic observation, is used to identify characteristics of the interactions between participants and the artifacts they create through sequential analysis of video-recorded concept development sessions. Though all parts of the CFCPing method described in previous sections were captured on video, including the Introduction, Breakout 1, Sharing of ideas, Breakout 2 and Presentation of solution concepts, this analysis focused on interactions that occurred during the small group work: Breakout 1 and Breakout 2. This was because these two parts of the collaborative session represent periods of un-facilitated small group activity and interdisciplinary interaction. The remainder of this section outlines the approach taken to develop and code video data collected during the collaborative sessions. Qualitative analysis of the responses to feedback questionnaires was also conducted. The findings from the questionnaires are presented and compared against the results of systematic observation and sequential analysis in the next section of this thesis.

\section{Systematic Observation}

According to Bakeman and Gottman (1997, p. 4) the two defining characteristics of systematic observation include (1) the use of a predefined coding scheme, and (2) that this coding scheme be applied by observers (or coders) of 'demonstrated reliability'. More specifically, when using systematic observation, "investigators need to say what they hope to find out" (research question/hypothesis) "define what seems important conceptually" (objects of analysis), "find ways to measure those concepts" (coding scheme) and "establish the reliability of their measuring instruments" (Bakeman \& Gottman, 1997). As presented in the Introduction, this research aims to examine the role 
of professional product designers and prototypes (visual representations/artifacts) in the context of cross-functional collaborative prototyping at the front-end of design development. The interactions between participants and artifacts, specifically visual representations are the objects of analysis. Following the example of Lewis, Mateas, Palmiter \& Lynch (1996, p. 2), a coding scheme was developed to include common events and interactions observed in the literature as well as events noted in initial review of the video-recordings. In addition, relevant information was extracted through an iterative process of watching video recorded group activities and noting 'instances' that were perceived and interesting/relevant to the observer, and identifying patterns in those instances (Wasson, 2000). The remainder of this section presents the coding scheme developed for this study, as well as the steps followed when coding data, which addresses observer reliability.

\section{Coding Scheme}

Three examples of systematic observation, identified in the literature, contributed to the development of the coding scheme used in this study. The first, by Robillard, d'Astous, Détienne \& Visser (1998) used coding schemes to identify and analyze behaviours exhibited by participants in their study of technical review meetings in software development. The second, by Lee, Popovic, Blackler \& Lee (2009) included a coding scheme for understanding knowledge management in user feedback sessions. The third is the previously mentioned study by Lewis et al. (1996), which includes a coding scheme process for using ethnographic data to drive product design.

According to Robillard et al. (1998) collaborative interaction should be evaluated in the context of both individual contributions and group interaction (social exchange), 
where individual contributions are defined by verbal utterance and an exchange as the smallest dialogue unit in an interaction when at least two speakers are contributing through verbal utterance. In the context of this study, individual contributions are identified as verbal utterances and physical actions and an exchange is identified as a series of related utterances and actions. Thus an initial coding scheme was developed to identify individual verbal and physical behaviours observed during small group interaction. Subsequent analysis of the coded data allowed for the identification of different types of exchanges between participants, which provide a more process-oriented outlook on group activity. The coding scheme for individual contributions is presented first, followed by the identified exchange types. Finally, an example of coded video data is presented showing all levels of coding.

\section{Coding scheme for individual contributions.}

To identify the role(s) played by professional product designers during collaborative concept development with nursing and pharmacy staff, the coding scheme includes characteristics of roles observed in literature related to group problem solving and facilitation (Schwartz et al., 2005) and collaborative design activities (Robillard et al., 1998; Lee et al., 2009; Lewis et al., 1996). For example the coding scheme developed by Lee et al. (2009) in their study of user-designer collaboration defined three levels of coding: (1) individual contributors including knowledge, identities and resources, (2) collective contributors which are akin to the exchanges identified in the coding scheme used in this study, and (3) designers' managerial activities. As this coding scheme was developed specifically for situations where designers had initiated and were leading the collaborative activity, e.g. user feedback sessions as part of product testing, it 
was not directly appropriate to the present study. As an example, this study does not presuppose or pre-assign management roles to designers or other participants, therefore some of the codes included in designers' managerial activities were rolled into individual contribution codes and others were not at all applicable to the CFCPing method.

To identify the role of visual representations and prototyping, the coding scheme included interactions between participants and artifacts, modeled after coding schemes used by Lewis et al. (1996, p. 52). Based on these references, the initial coding scheme included actors (participants), actions (physical and verbal behaviours) and artifacts (objects and concepts participants act upon) such that the full scope of individual behaviors could be captured and then examined in context. After initial video analysis, the coding scheme was revised and some specific action and artifact codes were added or eliminated to ensure mutually exclusive codes that accurately describe video content. One of the added codes was irrelevant verbal behaviour, the definition of which was modified from Kramer (2010). The final coding scheme for this study is presented in Table 2, including definitions for each actor, activity and artifact code used.

Table 2 .

Coding scheme to identify individual behaviours

\begin{tabular}{|c|c|}
\hline Codes & Definitions \\
\hline ACTORS & (2) \\
\hline \multicolumn{2}{|c|}{$\begin{array}{l}\text { (The \# identifies specific participants in a collaborative session when there is more than one } \\
\text { from the same disciplinary background) }\end{array}$} \\
\hline $\mathrm{N} \#$ & Nurse \\
\hline D\# & Designer \\
\hline $\mathrm{P} \#$ & Pharmacist \\
\hline PT\# & Pharmacy technician \\
\hline $\mathrm{F}$ & Lead facilitator (running the CFCPing sessions in this study) \\
\hline Team & $\begin{array}{l}\text { This actor code is used as a receiver in the case of broadcast } \\
\text { statements made to all members in the small group) }\end{array}$ \\
\hline
\end{tabular}




\begin{tabular}{|c|c|c|}
\hline & Definitions \\
\hline BEUA & IOURS & (2) \\
\hline \multicolumn{3}{|c|}{ Verbal behaviours } \\
\hline \multirow{2}{*}{ 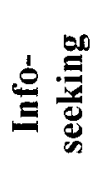 } & Question & $\begin{array}{l}\text { Information seeking: one participant asks a question to one or more } \\
\text { others }\end{array}$ \\
\hline & $\begin{array}{l}\text { Request } \\
\text { confirmation }\end{array}$ & $\begin{array}{l}\text { Information seeking: one participant asks a clarifying question to one } \\
\text { or more other participants based on a previous or ongoing discussion }\end{array}$ \\
\hline \multirow{5}{*}{ 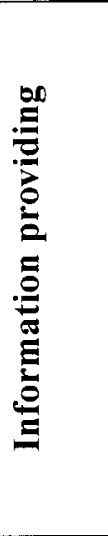 } & $\begin{array}{l}\text { Explain } \\
\text { (artifact) }\end{array}$ & $\begin{array}{l}\text { To give details of; make plain or intelligible; to clear (artifact) of } \\
\text { obscurity. As defined in the Oxford English Dictionary. }\end{array}$ \\
\hline & $\begin{array}{l}\text { Clarify } \\
\text { (artifact) }\end{array}$ & $\begin{array}{l}\text { To expand on or provide more detail about, or elaborate on an idea, } \\
\text { concept, statement, etc. previously raised by the participant or another. }\end{array}$ \\
\hline & $\begin{array}{l}\text { Relate to } \\
\text { (artifact) }\end{array}$ & $\begin{array}{l}\text { Characterized by use of an analogy or conjunction to relate the current } \\
\text { situation, topic or idea to previous experience, current context, or a } \\
\text { familiar object. }\end{array}$ \\
\hline & Agree & $\begin{array}{l}\text { Positive affirmation, confirmation or acceptance of a statement or } \\
\text { action made by another participant. }\end{array}$ \\
\hline & State & $\begin{array}{l}\text { Information provision: participant makes a statement, interjects, raises } \\
\text { a point or provides information that is not part of a question-answer } \\
\text { dialogue }\end{array}$ \\
\hline 竞离 & $\begin{array}{l}\text { Suggest } \\
\text { (artifact) }\end{array}$ & $\begin{array}{l}\text { Participant makes a suggestion in the form of a solution idea/concept } \\
\text { (idea), or the groups working style (plan). Could be in the form of a } \\
\text { question or statement, but should be coded as suggest only, not both. }\end{array}$ \\
\hline \multirow{4}{*}{ 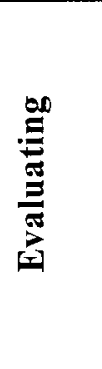 } & Reject & $\begin{array}{l}\text { A negative statement, criticism or disagreement with a previous } \\
\text { statement or idea. }\end{array}$ \\
\hline & Doubt & $\begin{array}{l}\text { Raising concern, expressing uncertainty with respect to a statement, } \\
\text { plan, or idea. Not as strong as rejection. }\end{array}$ \\
\hline & Evaluate & Assigning value to or appraising why an idea/situation is good/bad. \\
\hline & Justify & $\begin{array}{l}\text { To make good an argument, statement, or opinion; to confirm or } \\
\text { support by attestation or evidence. As defined by the Oxford English } \\
\text { Dictionary. }\end{array}$ \\
\hline \multirow{4}{*}{ 易 } & Coach & $\begin{array}{l}\text { Instance where one participant helps another participant in executing } \\
\text { an activity, or explains what they are doing by giving instruction on } \\
\text { how to execute an activity. }\end{array}$ \\
\hline & Order & $\begin{array}{l}\text { An instruction regarding individual or group problem solving or } \\
\text { design development activities. For example assigning a task to a } \\
\text { group member. }\end{array}$ \\
\hline & Summarize & $\begin{array}{l}\text { Participant re-states previous discussion/decisions. Arguments in this } \\
\text { instance might be 'problem', 'ideas', etc. }\end{array}$ \\
\hline & Irrelevant & $\begin{array}{l}\text { Comments, joking, or social conversation not related or contributing to } \\
\text { group activity, problem solving or design development. }\end{array}$ \\
\hline \multicolumn{3}{|c|}{ Physical behaviours } \\
\hline & $\begin{array}{l}\text { Gesture } \\
\text { (artifact) }\end{array}$ & $\begin{array}{l}\text { Gesture at or over an artifact, at one or more participants, or in the air. } \\
\text { If the gesture is not directed at or over an artifact (but in general, at a } \\
\text { person, or in the air) the argument for this behaviour code is blank. }\end{array}$ \\
\hline 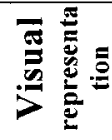 & Sketch & $\begin{array}{l}\text { To draw or sketch on a blank sheet or reference handout. } \\
\text { Characterized by sweeping pen strokes (drawing lines/circles) vs. } \\
\text { handwriting text. }\end{array}$ \\
\hline
\end{tabular}




\begin{tabular}{ll} 
Codes & Definitions \\
$\begin{array}{ll}\text { Make/modify, } \\
\text { (artifact) }\end{array}$ & $\begin{array}{l}\text { To produce/build/modify a tangible object. Refers to the development } \\
\text { of mock-ups and models. }\end{array}$ \\
$\begin{array}{l}\text { Write on } \\
\text { (artifact) }\end{array}$ & $\begin{array}{l}\text { Note-taking. Textual additions to reference material, sketches or } \\
\text { notepaper. }\end{array}$ \\
\hline $\begin{array}{l}\text { ARTIFACTS } \\
\text { Physical artifacts: (tangible objects) * these are props to support collaboration, what we are } \\
\text { interested in is how they fit into to group problem solving and we will answer this question by } \\
\text { identifying who, uses them, how, for what (intention) and to what end. }\end{array}$ \\
\hline $\begin{array}{ll}\text { Handout } \\
\text { floor plan }\end{array}$ & $\begin{array}{l}\text { Handouts provided to all participants depicting the current floor plan } \\
\text { of the unit and floor plan detail of the existing Medication Room }\end{array}$ \\
\hline $\begin{array}{l}\text { Handout } \\
\text { pictures }\end{array}$ & $\begin{array}{l}\text { Images provided to all groups including images of the existing MR, } \\
\text { available Automated Dispensing Cabinet (ADC) and record } \\
\text { management technologies (barcode scanning, etc). }\end{array}$ \\
\hline $\begin{array}{l}\text { Other/ } \\
\text { Notes }\end{array}$ & $\begin{array}{l}\text { Other document/paper/notebook/etc. including personal notebooks } \\
\text { introduced by participants }\end{array}$ \\
\hline Sketch & Drawing made during the session by participant(s) \\
\hline Model & $\begin{array}{l}\text { Mock-up or prototype made during the session by participant(s) } \\
\text { Provided or desired materials for sketching, drawing, making, } \\
\text { referencing. Some coding may include specific reference, for } \\
\text { example: materials (white paper) or materials (flipchart) }\end{array}$ \\
\hline
\end{tabular}

Conceptual artifacts (intangible: ideas, concepts) ${ }^{*}$ represent individual contributions in terms of knowledge of the problem, context and solution

Design requirement outlined in handout/presentation or acknowledged

$\begin{array}{ll}\text { Problem } & \text { by participants based on knowledge/experience. E.g. session } \\ \text { objectives, identified influencing factors that could impact medication }\end{array}$ safety, new problems identified in the current context, etc.

\begin{tabular}{ll}
\hline Ideas & Solution ideas/concepts \\
\hline $\begin{array}{l}\text { Personal } \\
\text { experience }\end{array}$ & $\begin{array}{l}\text { Previous experience in the current context, at another hospital/in } \\
\text { another (general/professional) context. }\end{array}$ \\
\hline $\begin{array}{l}\text { Current } \\
\text { context }\end{array}$ & $\begin{array}{l}\text { Existing MR design/layout, existing tools/process associated with } \\
\text { medication preparation. }\end{array}$ \\
Familiar & $\begin{array}{l}\text { An object or concept that is likely to be familiar or common } \\
\text { knowledge amongst group members, not specific to the hospital } \\
\text { environment. May be an analogy or metaphor to support explanation } \\
\text { of a concept or idea. }\end{array}$ \\
\hline & $\begin{array}{l}\text { A suggestion or idea related to the group's problem solving or } \\
\text { brainstorming activities. This could be an argument for 'order' in the } \\
\text { form of assigning a role/task to another participant, 'suggest'ing that } \\
\text { the group uses a certain strategy or approach, etc. This is a facilitative } \\
\text { activity. }\end{array}$ \\
\hline
\end{tabular}




\section{Coding scheme for types of exchanges.}

As discussed above, an exchange is defined by a collection of utterances that are related through behaviour and content. Five types of exchanges were identified in the collaborative sessions analyzed in this study: Information, Ideation, Evaluation, Management and Social. The exchange types are defined in Table 3 and an example of coded dialogue is presented in Table 4.

Table 3.

Types of exchanges

\begin{tabular}{|c|c|c|}
\hline Type & Code & Description \\
\hline $\begin{array}{l}\text { Information } \\
\text { exchange }\end{array}$ & INFO & $\begin{array}{l}\text { Characterized by information seeking (question and request } \\
\text { clarification) and information provision (explain, clarify, relate to, } \\
\text { statement). Often including content related to the current context of } \\
\text { IV morphine preparation and administration and personal } \\
\text { experience. Include physical actions supporting explanation } \\
\text { including write on, sketching and gesture (e.g. towards reference } \\
\text { material) supporting the documentation, transfer or translation of } \\
\text { information. }\end{array}$ \\
\hline $\begin{array}{l}\text { Idea } \\
\text { generation \& } \\
\text { elaboration }\end{array}$ & IDEA & $\begin{array}{l}\text { Characterized by the suggestion, clarification and positive } \\
\text { elaboration of solution ideas. In these exchanges, the coded } \\
\text { argument for the exhibited behaviour is 'idea'. Also includes } \\
\text { sketching and prototyping activities related to the embodiment of } \\
\text { ideas. }\end{array}$ \\
\hline $\begin{array}{l}\text { Evaluation \& } \\
\text { critique }\end{array}$ & EVAL & $\begin{array}{l}\text { Occurs when speakers critique design ideas and concepts contributed } \\
\text { by others or themselves. Characterized by the following behaviour: } \\
\text { reject, doubt, evaluate, and justify. The argument of coded verbal } \\
\text { behaviour is often 'idea'. Also includes sketching and prototyping } \\
\text { activities that support concept testing. }\end{array}$ \\
\hline $\begin{array}{l}\text { Management } \\
\& \text { leadership }\end{array}$ & MGMT & $\begin{array}{l}\text { Characterized by the suggestion of a plan, coaching or teaching by } \\
\text { one group member to one or more other group members, assigning } \\
\text { tasks (order) or summarizing ideas or plans. This behaviour } \\
\text { supports process management and structure of group problem } \\
\text { solving. }\end{array}$ \\
\hline Social & IRR & $\begin{array}{l}\text { Characterized by conversation that is unrelated to concept } \\
\text { development of the design task. Includes utterances coded as } \\
\text { (irrelevant), representing either 'jokes' or unrelated social } \\
\text { conversation. }\end{array}$ \\
\hline
\end{tabular}




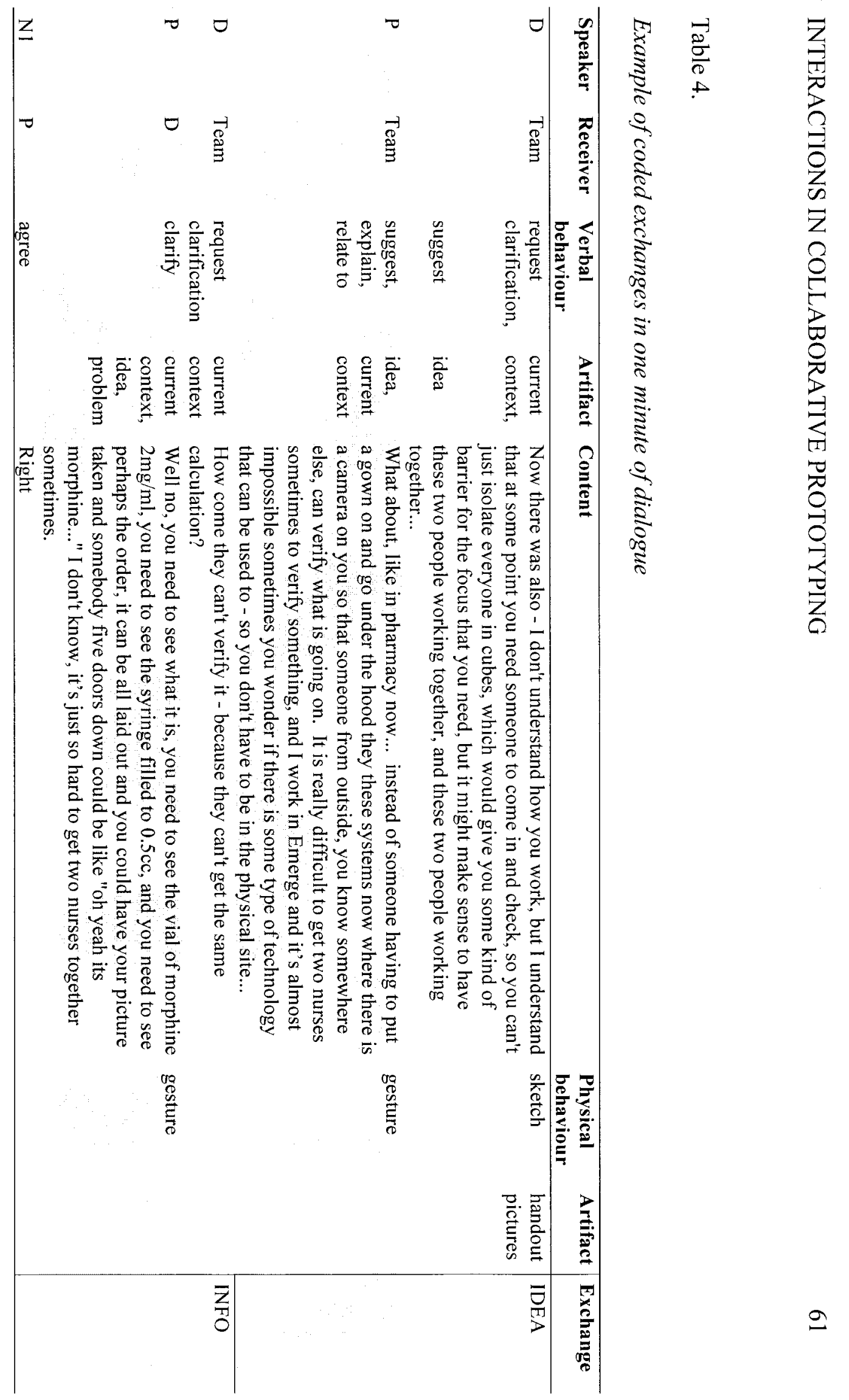




\section{Observer Reliability}

As mentioned above, observer reliability is one of the defining attributes of systematic observation and supports data reliability. When Lewis, Mateas, Palmiter \& Lynch (1996, p. 2) used Exploratory Sequential Data Analysis (ESDA) to analyze ethnographic videos in an effort to understand user needs in collaborative video editing from a user-interface design perspective, they devised a coding scheme and had three observers simultaneously review video footage, each looking for different elements: overall observations (including timing and demographic data), interactions, and events. The final coding keys were created from common events and interactions observed in the literature as well as events observed in initial sessions (Lewis et al., 1996, p. 2). A similar process was followed for the development of the coding scheme used in this study, albeit with one observer, instead of three. A coding strategy was developed during initial review of video clips, in an effort to minimize loss of data and strengthen reliability of a single observer. The coding strategy follows five steps:

1. Transcribe actors: 'To' 'From', code 'Verbal behaviours' and 'Elapsed Time', stopping the video regularly to ensure accurate capture of utterances. Enter 'Contents' based on a summary of participant's dialogue.

2. Review video and code physical behaviours. Verify verbal behaviour codes and elapsed time entries.

3. Review video a second time, verify all columns and document general observations.

Using this strategy the video coding to real-time ratio was 18:1. Meaning, it took approximately six hours to code and verify 20 minutes of video before starting formal 
analysis. This time estimate does not include coding of exchange types, which was part

of the formal analysis. An example of general observations documented by the observer

is presented in Figure 5.

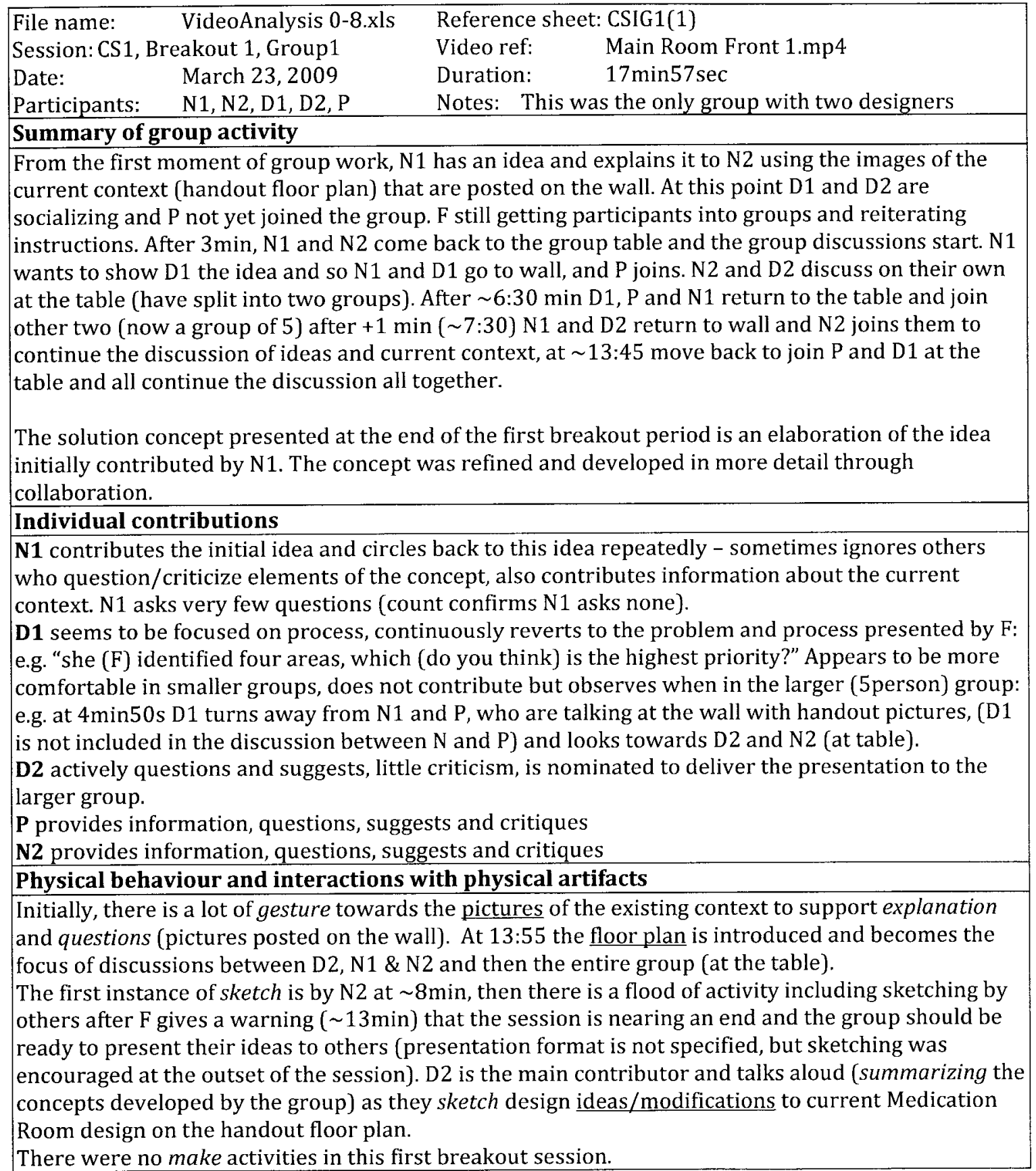

Figure 5. Example of general observations capturing small group behaviour 
INTERACTIONS IN COLLABORATIVE PROTOTYPING

\section{Results}

The results presented in this section are based on qualitative analysis of the video recorded collaborative sessions and feedback questionnaires. Following the coding scheme from the previous section, a description of small group behaviours is presented first, based on iterative coding and analysis of individual utterances (what people say) and actions (what people do). Identified patterns are compared between disciplines across small groups. Interaction between participants, based on coding and sequential analysis of exchange types, are presented second. Finally, feedback from participants and general observations related to the setup and materials provided for the sessions are presented with the results of questionnaire analysis.

\section{Description of Small Group Behaviour}

The following qualitative description of small group interaction was developed based on: (1) counting and calculating rates and frequencies of observed verbal and physical behaviours, and (2) general observations gleaned by the author through repeated viewing and coding of video recordings. An example of the preliminary analysis of a single group's behaviour is presented in Appendix 4. The remainder of this section describes small group activity based a comparison across groups.

Group activity was initially dominated by verbal communication. This is consistent with observations made in previous studies by Chung $(2009$, p. 87-88). In keeping with this initial observation, a comparison of verbal behaviours is presented first, followed by a description of the physical behaviours observed during interdisciplinary interaction. 


\section{Verbal behaviours by discipline across all groups.}

Conversation about current problems, requirements, previous experience and challenges were common topics of discussion amongst group members especially in Breakout 1, which represents approximately one third of total group work time. During this period, designers exhibited the majority of information seeking behaviour, asking questions and requesting clarification of the current context three times more than any other discipline. This is illustrated in Figure 6.

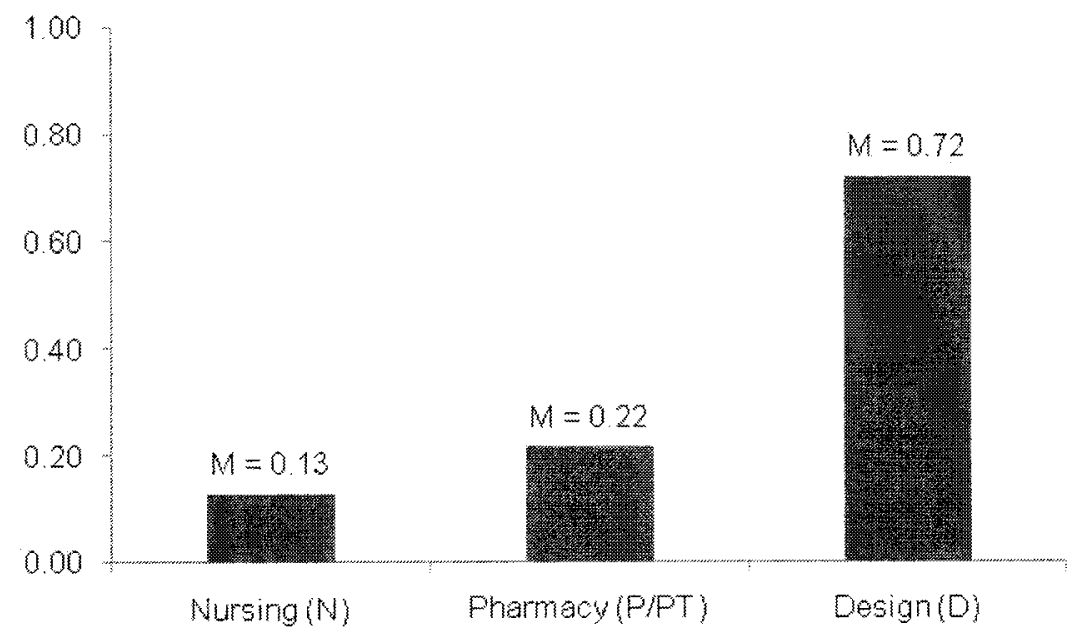

Figure 6. Average proportion of information-seeking behaviour (question and request confirmation) exhibited in Breakout 1

This is not surprising, as designers were less familiar with hospital practices and design issues than hospital staff that work regularly in the surgical unit context. Nurses and pharmacy staff provided explanations of current context, building on the information presented by the lead facilitator. Other explanatory behaviour included clarification of ideas and current context, and relating something to a familiar object or personal 
experience. Table 5 and Table 6 present examples of coded dialogue illustrating this type

of interaction.

Table 5.

Example dialogue including explanatory behaviour

\begin{tabular}{|c|c|c|c|c|}
\hline Speaker & Receiver & Verbal behaviour & Artifact & Dialogue \\
\hline $\mathrm{D}$ & Team $(\mathrm{T})$ & question & problem & $\begin{array}{l}\text { So, without breaching your own } \\
\text { confidentiality, when there is an } \\
\text { error...'cause I get the impression that it } \\
\text { is not giving morphine to the wrong kid, } \\
\text { its usually too much for that particular ... }\end{array}$ \\
\hline$P$ & D & explain & $\begin{array}{l}\text { current context } \\
\text { problem }\end{array}$ & $\begin{array}{l}\text { Sometimes when you have a teeny weeny } \\
\text { baby, its so easy to get mixed up between } \\
0.02,0.2 \text { and } 2 \text { and that's where we get } \\
\text { some of the } 10 \text { fold errors, and we've } \\
\text { averted some of that by going to the } \\
2 \mathrm{mg} / \mathrm{ml} \text { vials, but... such a wide } \\
\text { assortments of patient weights...there is } \\
\text { no real standard dose you're using }\end{array}$ \\
\hline $\mathrm{N} 1, \mathrm{~N} 2$ & $\mathrm{P}$ & agree & current context & $\mathrm{hmm}$ \\
\hline $\mathrm{N} 2$ & $\mathrm{P}, \mathrm{D}$ & agree, explain & current context & $\begin{array}{l}\text { That's right, because its all by weight, } \\
\text { once you're an adult and you're } 18 \text { or } \\
\text { older we don't really go by weight } \\
\text { anymore }\end{array}$ \\
\hline N1 & $\mathrm{D}$ & clarify & current context & $\begin{array}{l}\text { If you're over } 50 \mathrm{~kg} \text { then you sort of go } \\
\text { into this generalized category }\end{array}$ \\
\hline $\mathrm{N} 2$ & N1 & agree & current context & $\mathrm{hmm}$ \\
\hline
\end{tabular}

Table 6.

Example dialogue including explanatory behaviour

\begin{tabular}{|c|c|c|c|c|}
\hline Speaker & Receiver & Verbal behaviour & Artifact & Dialogue \\
\hline $\mathrm{P}$ & Team & suggest & idea & $\begin{array}{l}\text { I was thinking almost like a telephone } \\
\text { booth... like the nurse would go into this } \\
\text { telephone booth, this narcotic booth, } \\
\text { where you have the narcotic prep area }\end{array}$ \\
\hline $\mathrm{D}$ & $\mathbf{P}$ & agree & idea & Yeah, and its quiet \\
\hline $\mathrm{P}$ & Team & clarify & idea & $\begin{array}{l}\text { Even if you can't physically close the } \\
\text { door - }\end{array}$ \\
\hline $\mathrm{N}$ & Team & agree & idea & No I don't want it closed \\
\hline $\mathrm{D}$ & $\mathrm{P}$ & relate to & $\begin{array}{l}\text { familiar } \\
\text { concept }\end{array}$ & It's like the Dr. Doo booth \\
\hline $\mathrm{P}$ & $\mathrm{D}$ & agree & $\begin{array}{l}\text { familiar } \\
\text { concept }\end{array}$ & Yeah \\
\hline
\end{tabular}


These interactions appear to support the transfer of information and development of a common understanding of design requirements beyond that provided by the introductory presentation of the design problem. The personal experience of nurses grounded the design problem firmly in the current context. Similarly referencing a familiar concept, which is a type of shared knowledge, to explain solution ideas supports the transfer and translation of unshared knowledge, building the total shared understanding of the design problem and solution concept within the group.

All participants contributed ideas. Though in some groups one person may have contributed more ideas initially, as the session progressed the contribution and modification or contribution to the development of ideas was more evenly distributed between group members.

The majority of evaluative behaviours: reject, doubt, evaluate and justify, exhibited at the early stage were also by hospital staff, which are arguably more knowledgeable of environmental constraints, but also less likely to be familiar with the rules of creative brainstorming. Across all six groups, designers appear to avoid rejection and doubt in the early phases of concept development, while hospital staff expresses varying amounts of rejection and doubt. Observation suggests that this may be related to a lack of experience with creative brainstorming and the rules that were highlighted during introduction, including "no criticism" and "encourage wild ideas" (Osborn, 1963; IDEO, 1999). An example of evaluative behaviour is presented in Table 7 and Figure 7 presents the average number of each kind of evaluative behaviour expressed by discipline across five small groups. 
Table 7.

Example of evaluative behaviour

\begin{tabular}{|c|c|c|c|c|}
\hline Speaker & Receiver & $\begin{array}{l}\text { Verbal } \\
\text { behaviour }\end{array}$ & Artifact & Dialogue \\
\hline $\mathrm{P}$ & $\mathrm{N}$ & suggest & idea & $\begin{array}{l}\text { As a nurse if she could have a clear print out of } \\
\ldots\end{array}$ \\
\hline $\mathrm{N}$ & $\mathrm{P}$ & $\begin{array}{l}\text { doubt, } \\
\text { relate to }\end{array}$ & $\begin{array}{l}\text { idea, } \\
\text { current context }\end{array}$ & $\begin{array}{l}\text { Yeah but we're not getting that, even with } \\
\text { EClinDoc... }\end{array}$ \\
\hline $\mathrm{P}$ & $\mathrm{N}$ & agree, clarify & plan & $\begin{array}{l}\text { Agreed, that's something down the road, but we } \\
\text { can dream about it ... }\end{array}$ \\
\hline M & $\mathrm{N}$ & coach/teach & plan & Yeah we dream about it now \\
\hline
\end{tabular}

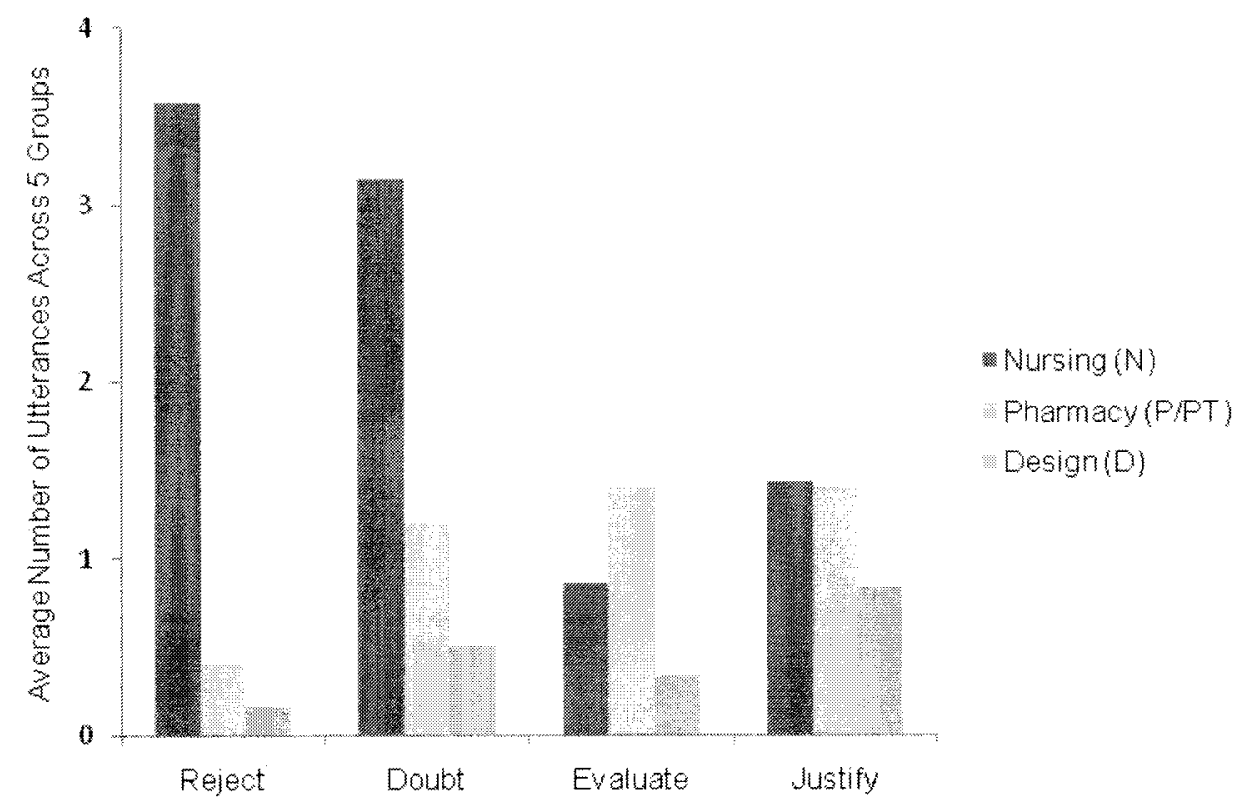

Figure 7. Average number of evaluative behaviours exhibited in Breakout 1

The next group of behaviours that was observed are related to the planning and organization of group activities: suggest(plans), coach/teach, order, and summarize. In most groups, designers made more suggestions than non-designers, with respect to the organization of group problem solving. An example is presented in Table 8. As the session progressed, hospital staff became more engaged in prototyping and making 
models of the ideas conceptualized by the group and also engaged in organizing group activities.

Table 8.

Example of coded exchanges

\begin{tabular}{|c|c|c|c|c|c|c|}
\hline Speaker & Receiver & $\begin{array}{l}\text { Verbal } \\
\text { behaviour }\end{array}$ & Artifact & Content & $\begin{array}{l}\text { Physical } \\
\text { behaviour }\end{array}$ & Artifact \\
\hline$\overline{\mathrm{D}}$ & $\mathrm{N}$ & suggest & plan & $\begin{array}{l}\text { So can I ask you - if you were going } \\
\text { to - so designers we do everything } \\
\text { visually right, could you highlight } \\
\text { the most used and the least used } \\
\text { areas on here }\end{array}$ & gesture & $\begin{array}{l}\text { handout } \\
\text { floor } \\
\text { plan }\end{array}$ \\
\hline $\mathrm{N}$ & $\mathrm{D}$ & $\begin{array}{l}\text { evaluate, } \\
\text { explain }\end{array}$ & $\begin{array}{l}\text { plan, } \\
\text { current } \\
\text { context }\end{array}$ & $\begin{array}{l}\text { Well the problem is there is are two } \\
\text { levels right, there is level one and } \\
\text { level two, because the counter space } \\
\text { here is even less used than some of } \\
\text { the shelf space, because the area - }\end{array}$ & & \\
\hline $\mathrm{D}$ & $\mathrm{N}$ & $\begin{array}{l}\text { request } \\
\text { clarification }\end{array}$ & $\begin{array}{l}\text { current } \\
\text { context }\end{array}$ & There's shelving here? & sketch & $\begin{array}{l}\text { handout } \\
\text { floor } \\
\text { plan }\end{array}$ \\
\hline $\mathrm{N}$ & $\mathrm{D}$ & clarify & $\begin{array}{l}\text { current } \\
\text { context }\end{array}$ & $\begin{array}{l}\text { There are shelves here with the } \\
\text { patient in room bins are all along, so } \\
\text { the area where patient one's bins are } \\
\text { there are four rooms over here but } \\
\text { then there are individual rooms }\end{array}$ & gesture & $\begin{array}{l}\text { handout } \\
\text { floor } \\
\text { plan }\end{array}$ \\
\hline D & $\mathrm{N}$ & order & & $\begin{array}{l}\text { So number one then, you can have } \\
\text { three or four areas }\end{array}$ & & \\
\hline
\end{tabular}

Verbal contributions labeled coach/teach includes incidents where one group member reminded another group member of the rules of brainstorming, encouraged group members to participate in ideation or visual representation of their ideas, or helped a group member to modify prototyping materials. The few instances of coach/teach that were exhibited during initial verbal discussions were related to the rules of brainstorming, as illustrated in Table 7. Many more instances were exhibited by designers in conjunction with prototyping activities that took place in Breakout 2. In contrast, the summary of problems and ideas was used frequently throughout small group activity as a means to confirm shared understanding amongst group members. This behaviour was 
also dominated by designers, except for one group (CS2G1) where the pharmacist in the group took a leadership role. This isolated case may have been due to specific personality combinations within the group. Personality is a known variable of small group performance in design tasks (e.g. Kim, Kim \& Wilde, 2008) that is outside the scope of this study. In summary, summarize and coach/teach behaviours were used to support group design activities and serve as tools that facilitate process management.

Conversation that was unrelated to the design activity and fell into none of the above categories was labeled irrelevant. Irrelevant behaviour includes social discussion and joking, and was observed rarely, but in some groups more than others. Social conversation occurred most often with prototyping activities when there was a division of labour between participants. For example, if one or two participants were silently engaged in making a prototype, those not involved in the making may enter into social exchange while waiting for the model to be made. Thus very few instances appear during Breakout 1 and more occur towards the end of Breakout 2 where the majority of prototyping took place. The occurrence of sketching and making activities over time is presented in the next section.

\section{Physical behaviour by discipline across all groups.}

Physical behaviours fall into two broad categories. The first is gesture, and the second is documentation of information and ideas through visual representations: note taking, sketching and prototyping. Note taking was coded as write on in the coding scheme used in this study. Figure 8 presents a bar graph of the average frequency of each different type of coded behaviour by discipline. When looking at the bar graph, it is important to remember that Breakout 1 was approximately half the length of Breakout 2 
in time. The following paragraphs describe the observed occurrence of such behaviours and their relationship to the verbal behaviours outlined previously.

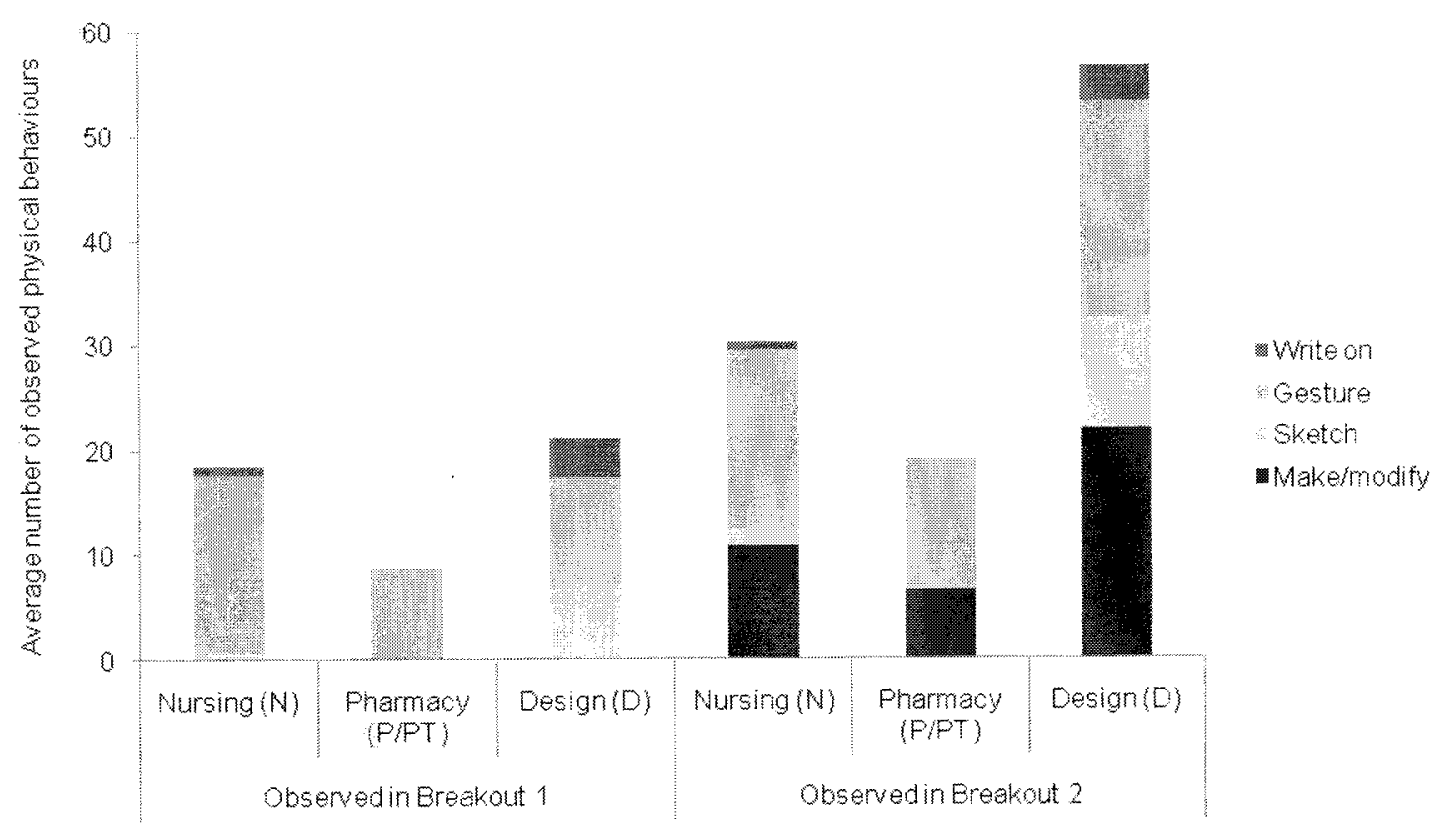

Figure 8. Average frequency of physical behaviours exhibited by discipline

Gesture. Gesture and demonstration of existing practices and tools, through reference to the pictures and floor plan diagrams provided, was observed frequently in early group interaction. As the session progressed, gestures were used to support the explanation and evolution of ideas. Observations across all six groups suggest designers and non-designers regularly used gesture to support verbal explanation and information transfer. For example, when explaining the size or shape of something or how it worked participants would essentially draw in the air to illustrate their point, as shown in Figure 9. 


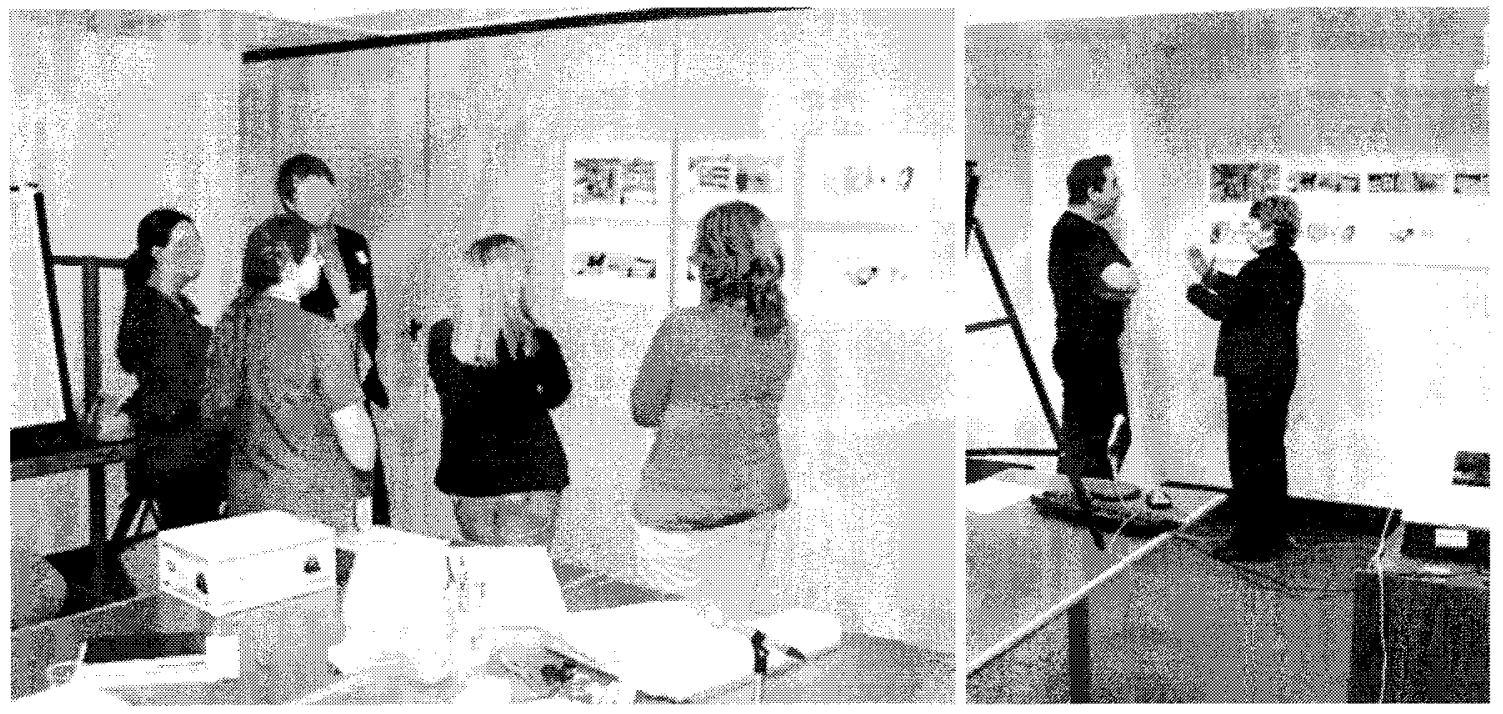

Figure 9. Using reference images and gesture to support information transfer

Overall, gesture was observed more frequently than any other verbal or physical behaviour and in both sessions, across all groups, non-designers gestured more frequently than designers. In fact, observations from this study suggest that non-designers are more likely to use gesture to illustrate an idea, which is an impermanent, momentary embodiment, than they are to modify a prototype or otherwise physically embody their idea.

Documentation. Documentation of information and ideas in the form of note taking was observed in some groups more than others. Across all groups, designers dominated note-taking behaviour. Sketching, as a means to explore and document ideas that had been raised by the group, was scarce in the initial phases of most group interaction, when verbal communication dominated group activity. As with note taking, sketching was used primarily by designers to document and test ideas expressed verbally by themselves or others, supporting communication of ideas between group members. 
Non-designers rarely participated in visual representation without encouragement, with the exception of one member of CS2G1 who was the only management representative that participated in the study. Typically non-designers would gesture at reference material, or at sketches produced by the designer(s) in the group, sometimes even with a pen in hand, but engaged less frequently in sketching or writing activities.

Encouragement to participate in visual representation came in many forms. For example, one designer instructed a nurse to prioritize high-flow or 'busy' areas on a floor plan of the surgical unit. In another case a designer led by example, using sketching as their primary explanatory and concept development tool. Pharmacy and nursing staff followed-suit when engaged in one-on-one discussions with this designer. In many cases, sketching appeared to serve as a means of dialogue with self and the design concept(s) in question. For example, in CS2G1 the designer would frequently sketch after other team members presented ideas and continue to sketch through verbal clarification and development of ideas by the group. Images representing typical use of sketching are presented in Figure 10.
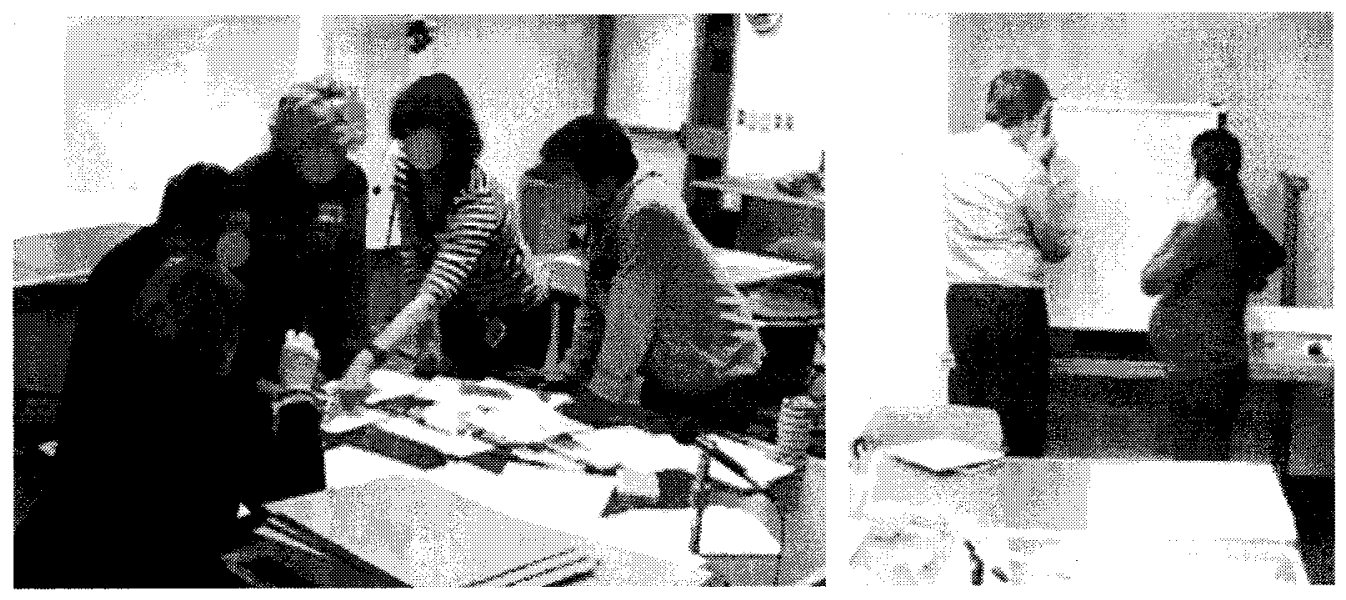

Figure 10. Examples of sketching and interaction with visual representations 
There was only one instance of prototyping in Breakout 1. In this instance a nurse folded a piece of paper to illustrate and explain the functionality of a tool used in the current context, the existing paper-based Medication Administration Record (MAR), as shown in Figure 11. In Breakout 2, after groups had an opportunity to share ideas engage in a short coffee break, more sketching and prototyping was observed.

In general prototyping was used to realize concepts that had already been discussed and support development of new ideas. In every group designers initiated model making, and were the first to modify the prototyping materials provided, cutting and shaping cardboard and foam to build representations of ideas that had been conceptualized by the group. Prototyping was also used for ideation and testing alternatives. For example, one designer initiated prototyping by playing with a piece of paper to test different forms for a 'narcotic booth' that had been conceptualized by the group. This act motivated nursing and pharmacy staff to engage in model making and resulted in two non-designers becoming the self-appointed primary model makers for the group. In other groups, designers did most of the material modifications, and nondesigners seemed to be more comfortable interacting with built prototypes by rearranging pieces or suggesting modifications and alternatives, as shown in. For example, in one group a pharmacy technician used part of the developing prototype to support explanation and testing of alternative ideas. The original concept was modeled after a room divider, as a visual barrier separating the narcotic preparation area from the rest of the medication room. The pharmacy technician proposed a shorter wall, height was discussed and the final decision was to have a full height wall with a frosted glass window. A series of 
images showing participants interacting with prototypes, including the previous example, are presented in Figure 11, Figure 12, and Figure 13.

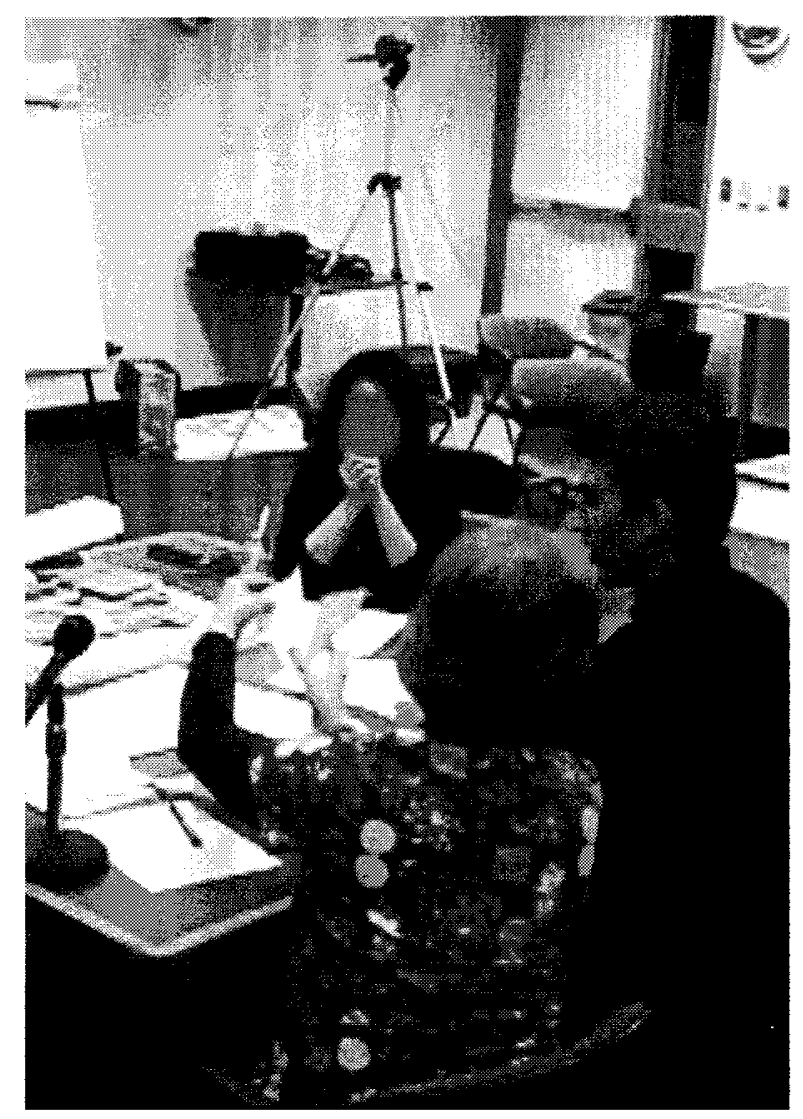

Figure 11. Nurse makes model of existing MAR to explain concept to designer

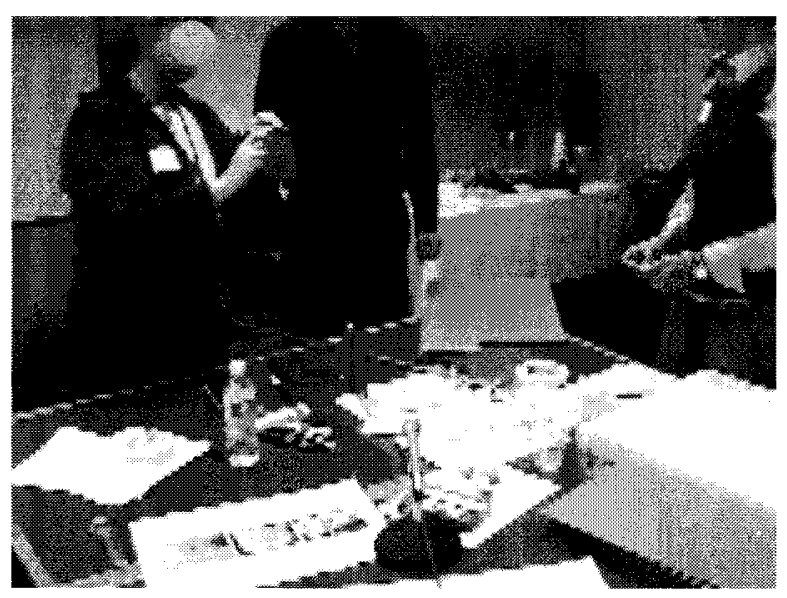

Figure 12. Pharmacy technician uses a piece of the model to generate new ideas 


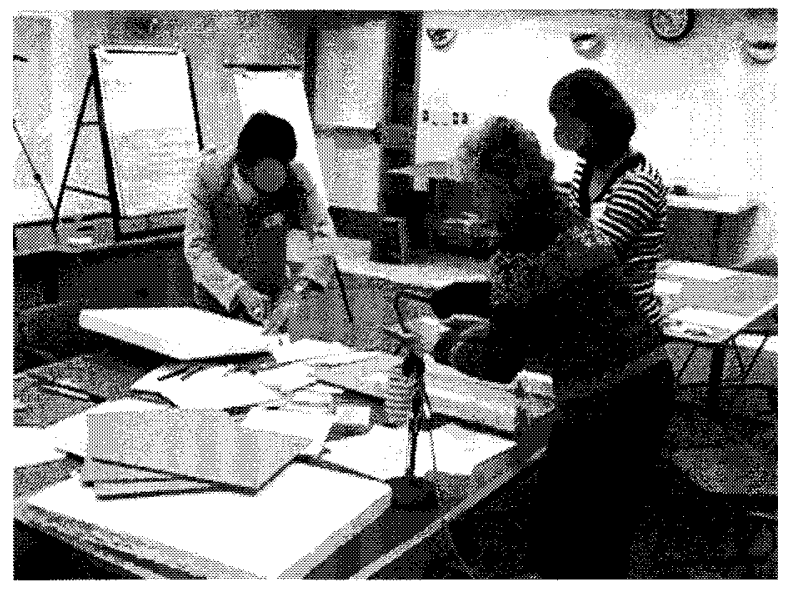

Figure 13. Designer makes new elements of the model while hospital staff interacts with the existing model

\section{Participation and group composition.}

The previous descriptions of verbal and physical behaviour examined both the relative frequency of different types of behaviour observed throughout the collaborative session, as well as the contributions made by participants across disciplines, as a function of the different types of individual contributions made by participants. The following discussion examines participation across disciplines using the total number of verbal interactions as an indicator of the contribution made by each group member.

Participation appears to be more or less distributed between group members, except in the two groups of five where there is one participant who is significantly less involved than others. Figure 14 illustrates the relative contributions made by each participant as a function of the total number of verbal interactions in each group. 


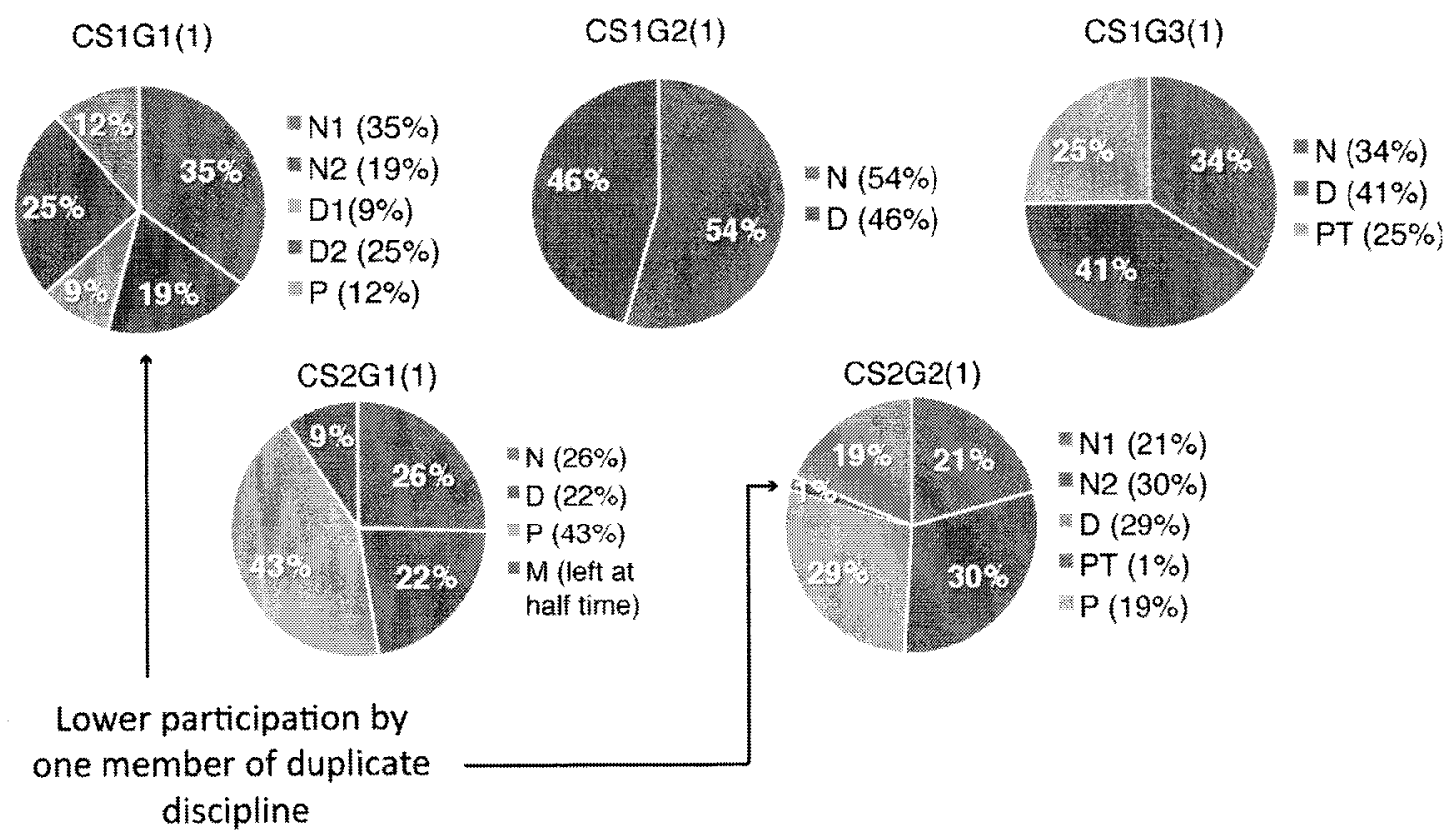

Figure 14. Relative participation by discipline in Breakout 1

In the groups of five, there were multiple participants from the same discipline.

For example in CS1G1 there were two designers, two nurses and one pharmacist. One of the designers participated in significantly less group discussion than the other team members. In CS2G2 there were two nurses, a pharmacist and a pharmacy technician. The pharmacy technician contributed significantly less than the pharmacist. This did not appear to be the case with respect to nursing representation, and was not observed in the two groups of four, one of which also had both a pharmacist and a pharmacy technician. Notes that CS2G3 is not represented in the figure because damaged audio prevented coding verbal contributions. In the two groups where there was more than one nurse, they both participated at similar levels. This may be related to their position as primary users of the existing and yet to be designed system.

The two five-person groups also repeatedly self-organized into sub-groups of two and three and then re-united as a larger whole. This pattern was repeated throughout the 
session: four times for CS1G1 and three times in CS2G2. Sometimes information was formally shared between sub-groups when the groups reunited, other times the same discussion would end up being repeated with a participant who was not involve in the discussion the first time. For example, Figure 15 shows the formation of sub-groups within CS2G2 during Breakout 2. In this example three hospital staff are focused on making parts of the model that have already been decided on, while the designer and a nurse discuss traffic flow through the room and generate alternate ideas for medication room layout. Figure 16 depicts the organization of group collaboration during Breakout 1 for CS1G1.

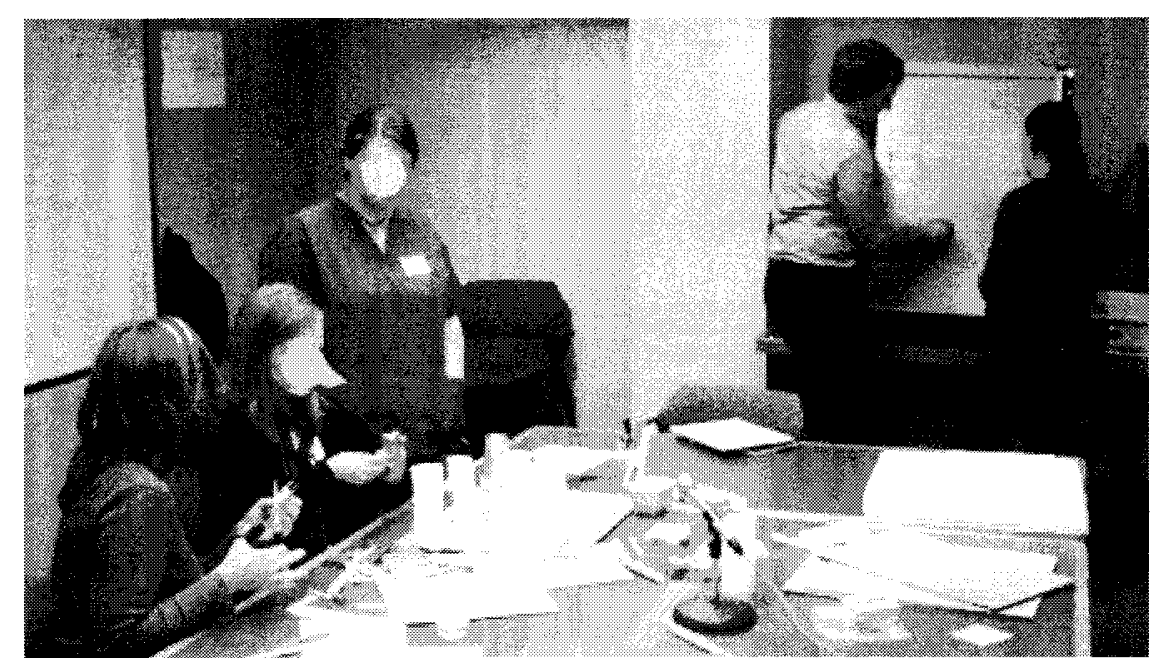

Figure 15. A group of five self-organizes into two smaller groups 


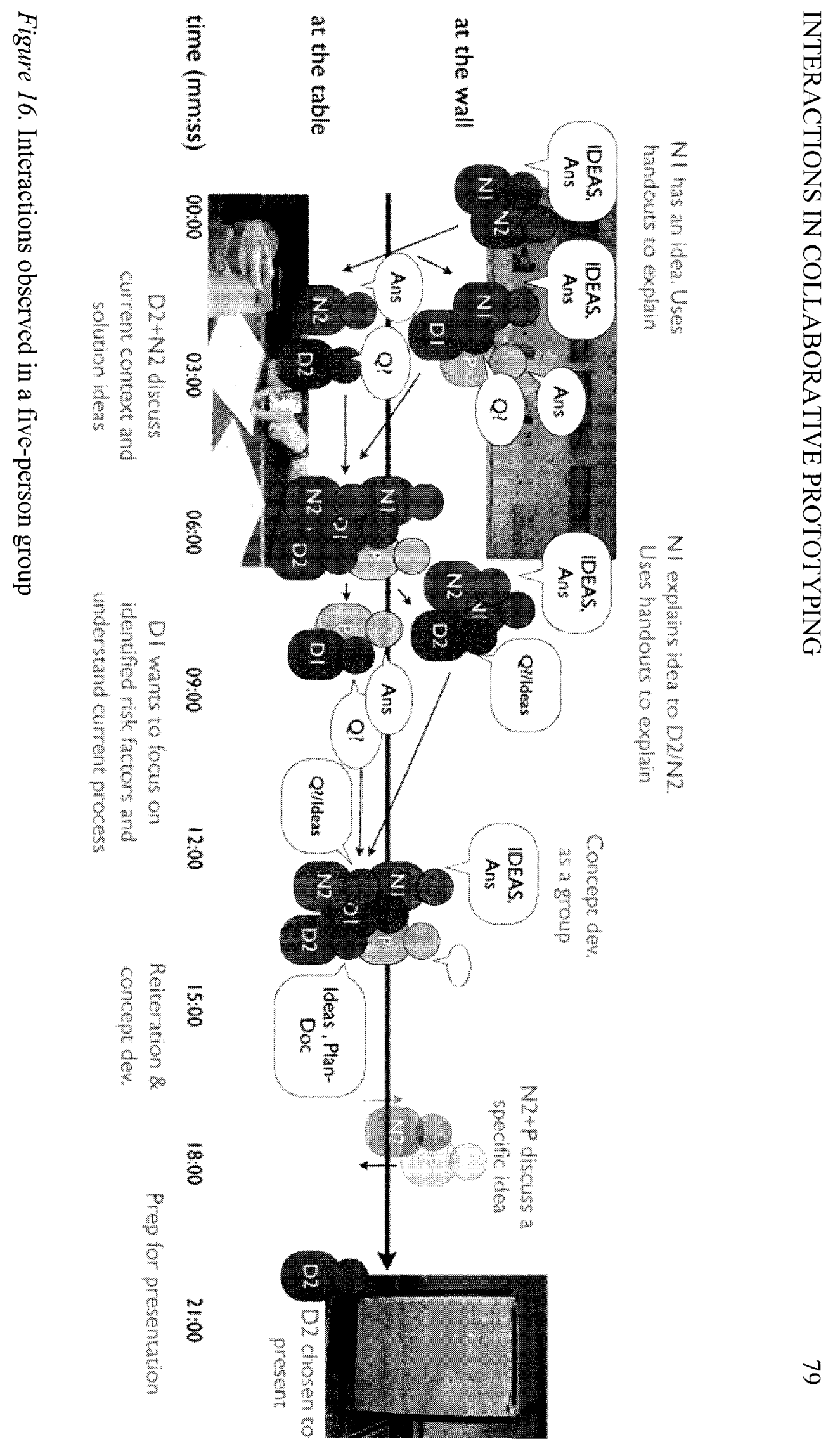




\section{Summary of observed behaviours.}

In summary, conversation dominated Breakout 1 and sketching was used to embody initial ideas generated by small interdisciplinary groups. Prototyping was used in the Breakout 2 to make models of the initial concepts generated in Breakout 1 and to further develop these concepts by supporting testing and generation of alternatives. Though all disciplines contributed to ideation and concept development, designers exhibited more coach/teach behaviour and were more likely to engage in organizing group activity. Conversely, non-designers provided more explanation and examples of the current context and design problems, and were more likely to critique or reject ideas presented by others. Designers encouraged non-designers to participate in sketching and prototyping either explicitly or leading by example. The relative contributions of verbal and physical behaviour made by each discipline suggest that designers and non-designers assumed different roles throughout the collaborative sessions. Specifically, designers were more likely to occupy a facilitative role, both with respect to the organization of group problem solving activities and by providing guidance and encouragement to nondesigners engaged in sketching and prototyping. Furthermore, using the total number of verbal contributions as a measure of participation reveals that in groups with more than four members and more than one representative from the same discipline one person contributed noticeably less than others. This suggests that duplication of disciplinary representation within one group may be less efficient.

In an effort to develop a better understanding of interdisciplinary interactions in CFCPing, the next section moves beyond individual contributions to examine types and sequences of exchanges between participants. 


\section{Types and Sequence of Exchanges}

As suggested by Robillard et al. (1998) interaction should be examined both as a function of individual contributions, discussed in the previous section, and exchanges between participants, which is the focus of this section. The coding scheme used in this study defined an 'exchange' as a series of sequential verbal and physical behaviours that are related through content. Thus the video transcripts, which had been previously coded for individual behaviour, were subsequently coded in terms of the following types of exchanges: Information (INFO), Ideation (IDEA), Evaluation (EVAL), Management (MGMT), and Social (IRR). An example of one minute of coded dialogue was presented

in Table 4. Analysis of the coded exchanges was used to identify the frequency and order in which different types of exchanges occurred within each group.

Considering the previous analysis of individual behaviours revealed variations in the type of behaviours exhibited over time (e.g. more information seeking/providing and no prototyping in the first breakout) the analysis of exchanges also included comparisons between Breakout 1 and Breakout 2 and across groups. Furthermore, as suggested by the analysis of individual contributions, very few social exchanges were observed. Though social exchange and the relationship between team members has been identified as a factor in the performance of teams within organizations (Wheelan, 2001), and literature suggests this supports the progress of groups through developmental stages (Argote, Gruenfeld \& Naquin, 2001), in CFCPing participants are selected based on disciplinary expertise, are more likely to have little prior knowledge of one another, and are required to quickly coordinate and develop solution concepts. Therefore, due to the low frequency of social exchanges and the indirect link between social exchange and knowledge 
management within the small ad-hoc groups in CFCPing, this type of exchange was eliminated from further analysis. The results of the analysis of interaction based on the identification of exchange types are presented in the following paragraphs.

First, a comparison of the relative proportion of exchange types within small group reveals that two of the groups (CS1G1 \& CS2G2) spent more time on ideation and evaluation, and the two other groups (CS1G2 \& CS1G3) exhibit a higher proportion of information and management exchanges. Recall that the remaining two groups (CS2G1 \& CS2G3) could not be part of this analysis due to missing video data. This is illustrated in Figure 17, which presents the relative proportion of exchange types exhibited by each of the four groups.
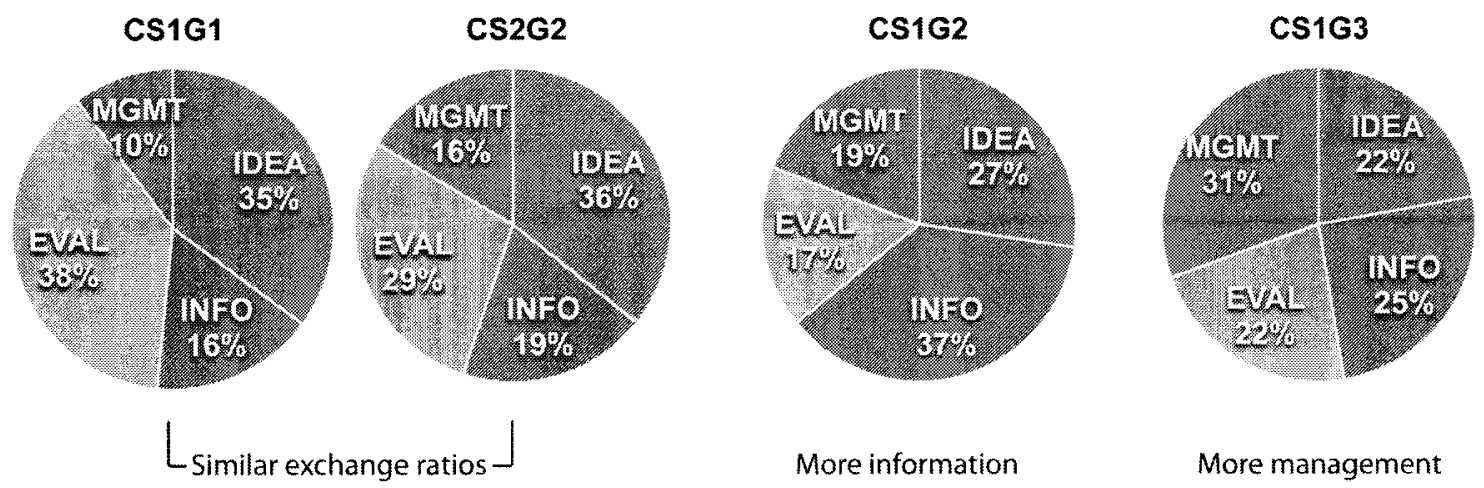

Figure 17. Proportion of group activity as a function of exchange type

Second, first order sequential analysis diagrams presented in Figure 18 and Figure 19 show that there are two emergent approaches to brainstorming and concept development, which can be associated with the patterns identified above. Two out of four groups exhibit the first approach, labeled Process1. These groups exhibit frequent transitions between ideation (IDEA) and evaluation (EVAL), depicted by thicker lines between exchange types, and less frequent transitions between Ideation and Information, and Evaluation and Management (MGMT). This approach is also characterized by 
infrequent transitions (very thin lines) between Information and Management, as shown in Figure 18. The groups exhibiting Process 1 are also those that exhibit an overall greater proportion of group activity focused on Ideation. The second approach, Process 2, exhibited by the other two groups is characterized by less frequent transitioning between Ideation and Evaluation and more between Information and Management.

The frequency with which transitions occurred is represented by the transitional probabilities indicated on the diagrams. For example, in Process 1 the number 0.63 beside the arrow pointing from IDEA to EVAL is the probability that an Ideation exchange will be followed by an Evaluation exchange. This is based on the average probability calculated across the groups that exhibited a similar process and represent the total number of exchanges exhibited across Breakout 1 and Breakout 2.

Further analysis, including the calculation of transition probabilities for each breakout separately, reveals that the groups exhibiting Process 2 do so for two different reasons. First, CS1G3 exhibited comparatively frequent transition between Information and Management in Breakout 1 as compared to all other groups. Second, CS1G2 exhibited comparatively infrequent Evaluation in Breakout 2 as compared to all other groups.

The sequential analysis diagrams presented in Figure 18 and Figure 19 also present the relative proportion of sketching and prototyping exhibited across exchange types. For example, in Process 1 the proportion of prototyping that occurred with exchanges between Ideation and Evaluation is shown as $0.35 \mathrm{~s}$. Looking at these diagrams it becomes clear that the groups exhibiting Process 1 used more sketching (s) 
and prototyping (p) to test and develop solution concepts, which is illustrated by a higher proportion of sketching and prototyping occurring between IDEA and EVAL.

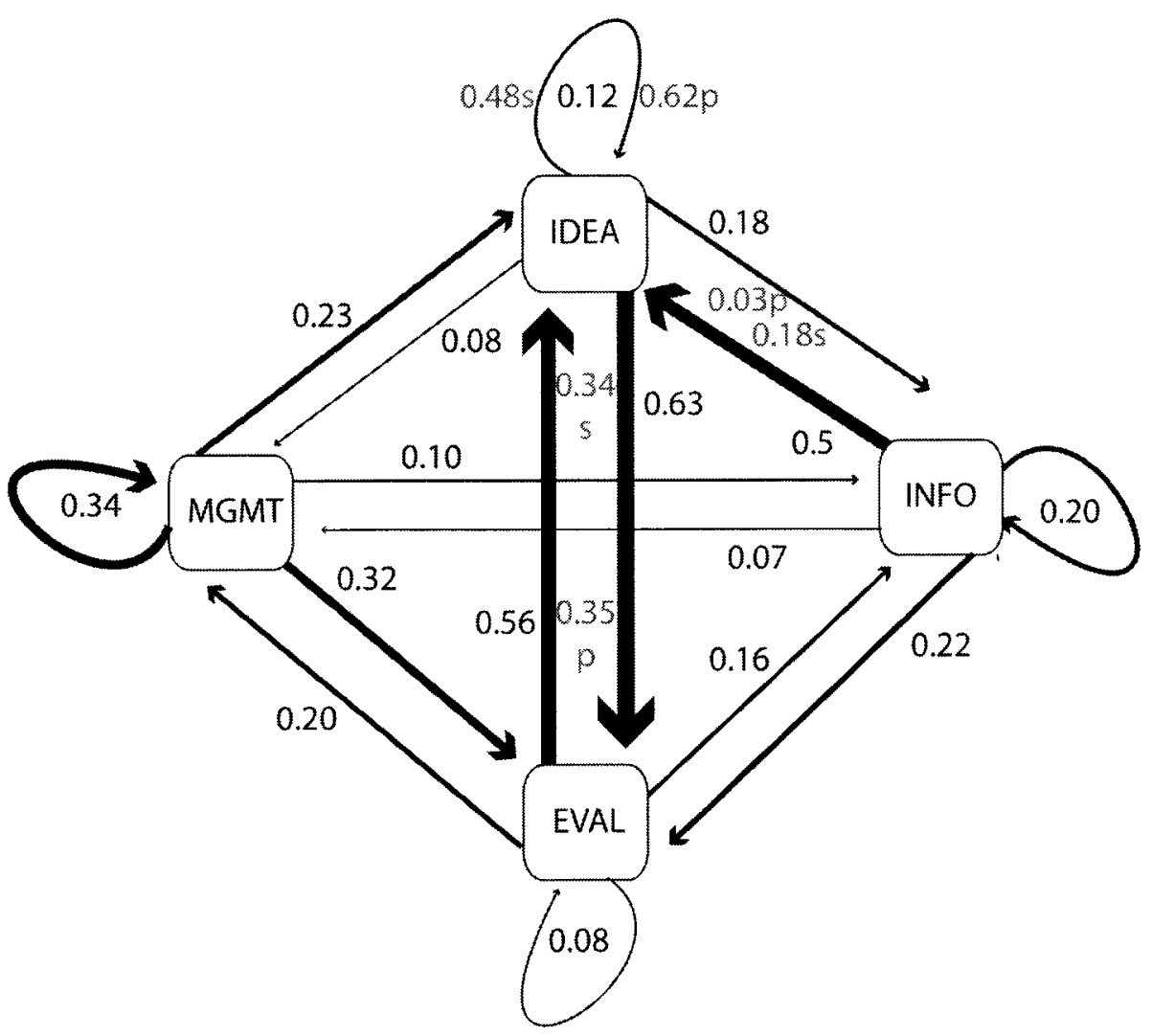

Figure 18. Emergent concept development Process 1 exhibited by CS1G1 \& CS2G2.

Thicker lines indicate more frequent transitions between exchange types, transitional probabilities are indicated beside each line, and grayed numbers indicate the proportion of sketching (s) or prototyping (p) observed during specific exchanges. 


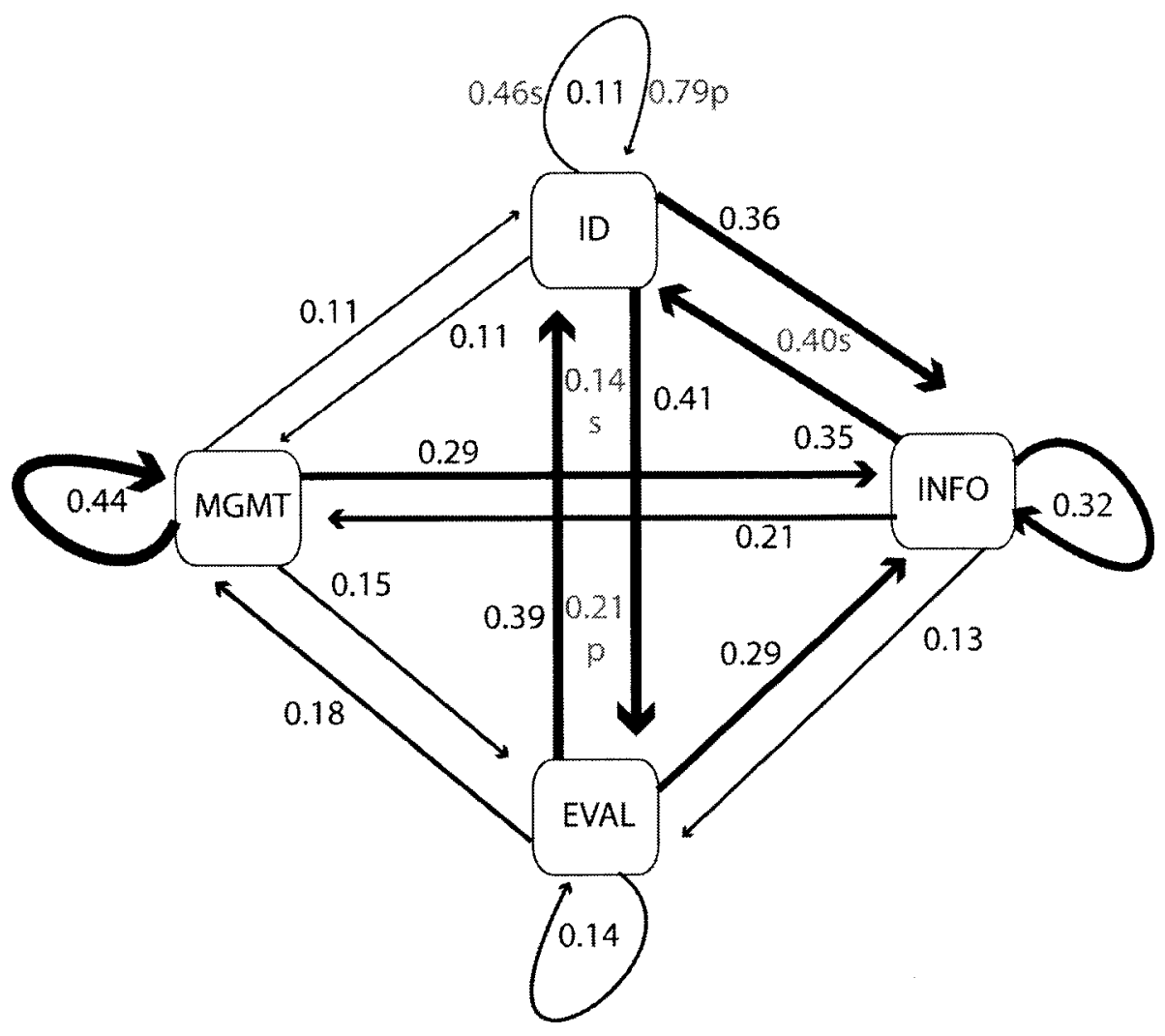

Figure 19. Emergent concept development Process 2 exhibited by CS1G2 \& CS1G3.

Thicker lines indicate more frequent transitions between exchange types, transitional probabilities are indicated beside each line, and grayed numbers indicate the proportion of sketching (s) or prototyping (p) observed during specific exchanges.

\section{Relating Interaction to Outcomes}

Further analysis of video data revealed that findings from both the analysis of individual contributions and exchanges between participants could be linked to outcomes. The outcome measure used to identify this relationship is the total number of ideas generated by each small group, presented in Table 9. The final two columns show the number of ideas adjusted as a function of the number of group members. As some participants left before the end of the session, the adjusted group size is based on the 
amount of group activity for which participants were present. From this table it appears that the number of group members present at the beginning of the session has a positive impact on the number of ideas generated by the group. Qualitative analysis of video data suggests that in most groups the ideas generated in Breakout 2 were reiterations or modifications of ideas originally presented during Breakout 1.

Table 9.

Number of ideas vs. group size

\begin{tabular}{lcccccc}
\hline Group & $\begin{array}{c}\text { \# General } \\
\text { ideas }\end{array}$ & $\begin{array}{c}\text { \# Specific } \\
\text { ideas }\end{array}$ & $\begin{array}{c}\text { Total } \\
\text { ideas }\end{array}$ & $\begin{array}{c}\text { Group } \\
\text { size }\end{array}$ & $\begin{array}{c}\text { Adjusted } \\
\text { Group } \\
\text { size }\end{array}$ & $\begin{array}{c}\text { Adjusted } \\
\text { Total } \\
\text { ideas }\end{array}$ \\
\hline CS1G2 & 6 & 3 & 9 & 2 & 2 & 4.5 \\
CS1G3 & 5 & 6 & 11 & 3 & 3 & 3.7 \\
CS2G3* & 7 & 13 & 20 & 4 & 4 & 5 \\
CS2G2 & 8 & 16 & 24 & 5 & 5 & 4.8 \\
CS1G1* & 10 & 19 & 29 & $5 \rightarrow 3$ & 4.25 & 6.8 \\
CS2G1* & 10 & 19 & 29 & $4 \rightarrow 3$ & 3.2 & 9.06 \\
\hline
\end{tabular}

* Group activity started with ideation (vs. information exchanges)

In addition, amongst the groups that generated more than 20 ideas are those that exhibited Process 1 . The groups that generated the lowest number of ideas exhibited Process 2, and spent noticeably more time in Information and Management type exchanges than the other groups, as shown in Figure 17. Further analysis of exchange types over time confirms that CS1G3 spent a lot of time in Management and Information transfer during Breakout 1, and did not start generating ideas until the end of Breakout 1. Looking back to the analysis of individual contributions reveals that this group exhibited more information seeking behaviour and fewer ideas during this breakout, than other groups. This may suggest that too much structure or planning hinders the productivity of brainstorming sessions. With respect to the second group that exhibited Process 2 and 
generated fewer ideas (CS1G2), the only group with two group members, analysis reveals very little transition between Ideation and Evaluation in Breakout 2. This group did not develop ideas through iteration and evaluation and focused mainly on embodiment of previously identified ideas, and information and planning. This underscores the importance of observations in previous research (Muller, 1992; Chung, 2009) that simple material facilitates making models and allowing groups more time to focus on evaluation and testing. It also suggests that three or more group members may lead to more evaluation and development of concepts.

\section{Summary of Video Analysis Results}

In summary, counting and comparison of verbal and physical behaviour exhibited between disciplines supports the identification of disciplinary roles in communication within groups. Subsequently, a qualitative analysis of individual contributions led to the identification of five types of interaction between participants. These exchanges, defined by a collection of related behaviours, include: Information, Ideation, Evaluation, Management and Social. Sequential analysis of identified exchanges indicates two distinct problem-solving processes exhibited by the observed groups. Process 1 is defined by frequent transitions between ideation and evaluation and infrequent transitions between information and management. Process 2 is defined by frequent transitions between information and management. The small groups exhibiting Process 1 generated more ideas than the groups exhibiting Process 2. Therefore, patterns in communication, based on the analysis of individual behaviours, lead to the identification of exchange types and sequential analysis of exchange types supports the identification of patterns in the interactions between participants within groups. Both communication and interaction 
patterns appear to impact outcomes. Thus the framework presented in Figure 20 is used to illustrate the relationship between communication and interaction patterns observed within groups and how these patterns can impact outcomes.

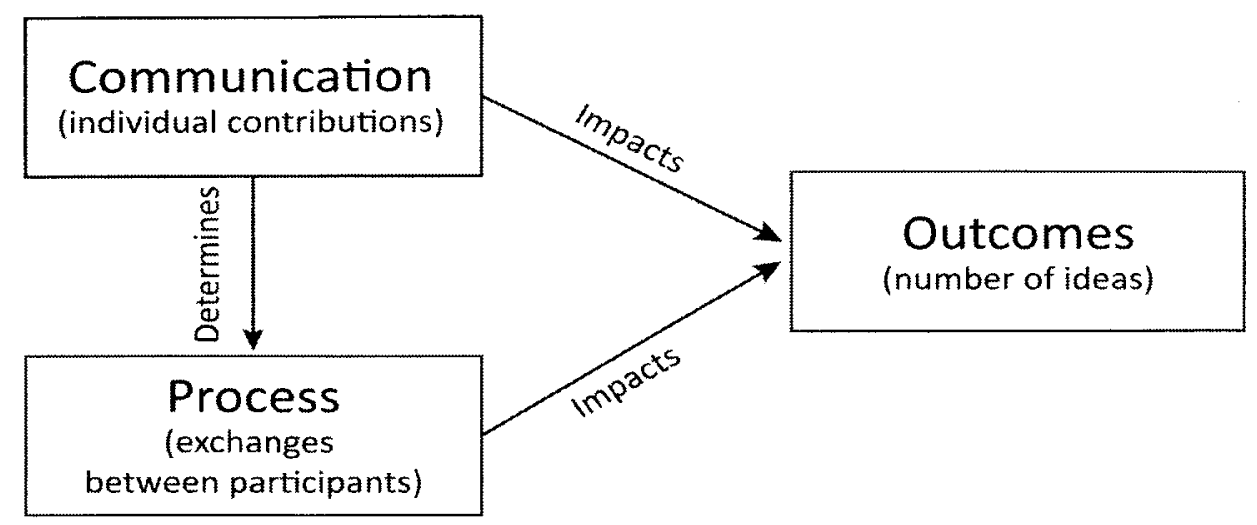

Figure 20. Framework describing study results

These results are based on analysis of coded behaviours observed during small group interaction. As such, they tell only part of the story. The following section provides another perspective based on participants' perceptions of the collaborative prototyping session, revealed through responses to the feedback questionnaire.

\section{Feedback From Participants}

The objective of the questionnaire was to identify participants' perception of the collaborative prototyping session with respect to: (1) the use of prototypes to develop solution concepts and (2) their role and the role of other team members in the collaborative activity. Questionnaire analysis was aimed at exploring differences in perception between disciplines and possible differences in perception between sessions in light of minor changes made between sessions (e.g. the Pictionary warm-up game was excluded in the first session). Consequently, analysis was conducted across disciplines and between sessions. The results are presented according to the grouping of related 
questions on the questionnaire itself: the CFCPing method, group dynamics and general impressions. When reading these results it is important to remember that the majority of questions were scored on a Likert scale of one to five, where one was defined as very negative and five very positive.

\section{Feedback on session structure and activities.}

The purpose of the questions in this section was to evaluate participants' perceptions of the CFCPing method, specifically how the activity of sketching and prototyping were perceived to impact group problem solving and design development.

Respondents across all disciplines agreed that the objectives of the session, as explained by the researcher during the introduction, were clear $(M=4.1$ for CS1 and $M=$ 4.5 for CS2) and that the video clip was a good introduction to the collaborative prototyping method $(\mathrm{M}=4.9$ for $\mathrm{CS} 1$ and $\mathrm{M}=4.7$ for $\mathrm{CS} 2)$. Hospital staff across both sessions found the video motivating. This suggests that the video effectively introduced the collaborative prototyping method to non-designers. One group of designers was less motivated by the video (assigning scores of three out of five), possibly because they may have been very familiar with it. A clip of the IDEO Deep Dive video (ABC News, 1999) was originally selected because it is a popular example of collaborative design process.

The warm-up game, intended to encourage non-designers to participate in sketching and making activities was not conducted during the first collaborative session because of time pressures. One participant indicated that "Extra time for info (no Pictionary) was good - this is a very complex issue being touched on in a bare minimum of time". In the second collaborative session the perceived benefit of this activity varied 
from positive to indifferent across disciplines, as shown in Figure 21. This question received the lowest average score across survey respondents at $\mathrm{M}=3.45$ out of 5 .

Was the pictionary game a good warm up to sketching and prototyping with your team?

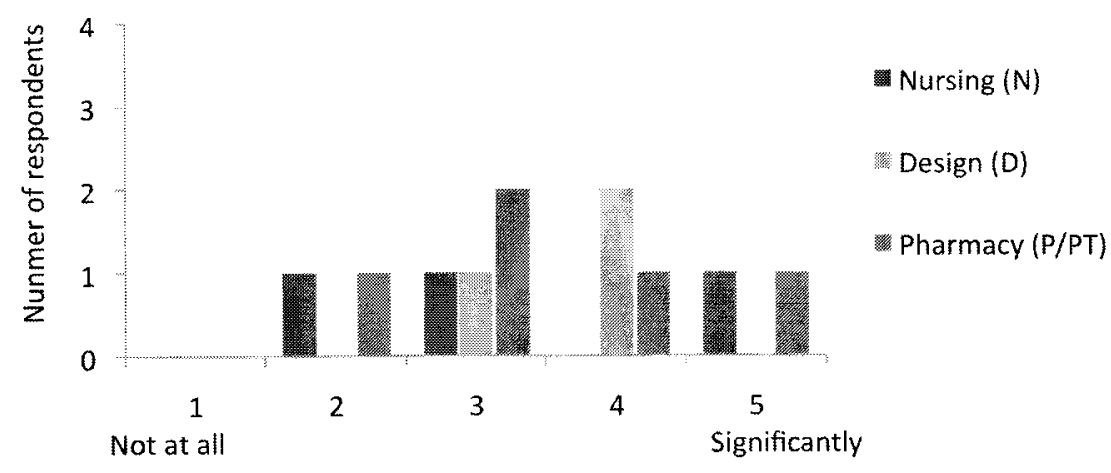

Figure 21. Feedback from participants on the Pictionary warm-up game

It appears that the inclusion or exclusion of this particular warm-up exercise did not have a great effect on the comfort level of participants, specifically non-designers, with respect to sketching and prototyping activities. Overall, respondents indicated they were comfortable both sketching and prototyping and there was no great difference in scores between disciplines, as illustrated in Figure 22.

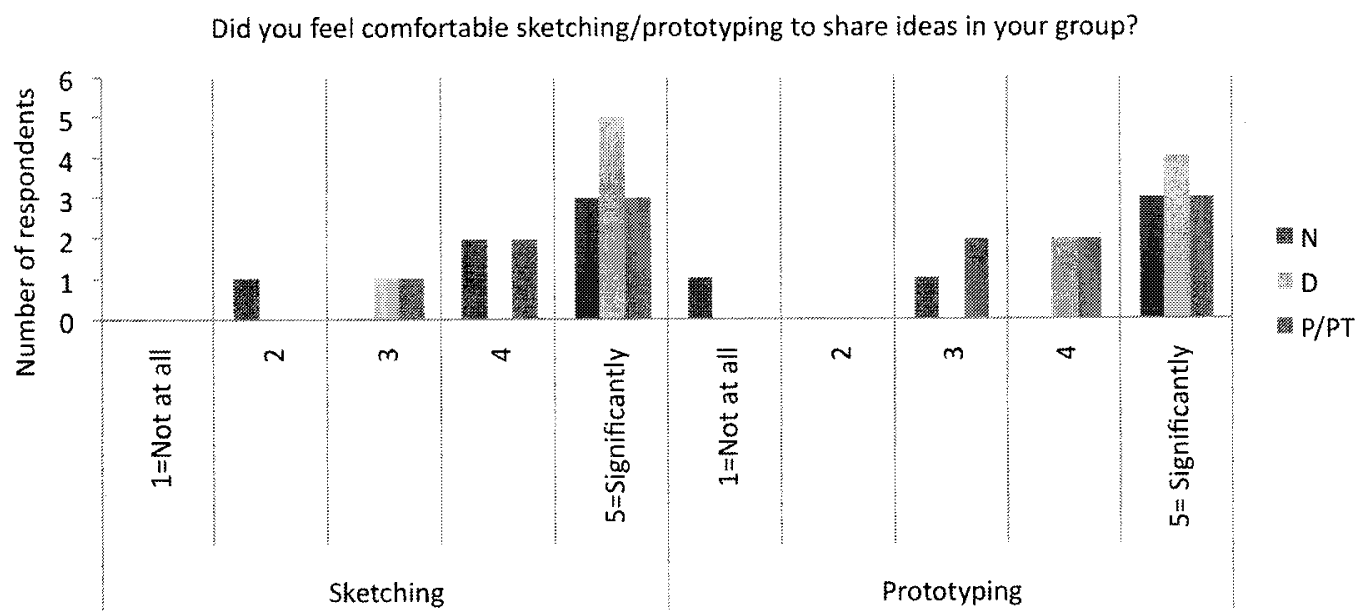

Figure 22. Feedback from participants on the use of sketching and prototyping 
Previous research (Muller, 1992; Chung, 2009) suggests that non-designers may be less likely to interact with prototyping materials or make three-dimensional visual representations of their ideas because the medium is unfamiliar to them. One of the observations by Chung (2009) regarding non-designer participation in collaborative prototyping was that non-designers seemed hesitant to make, but would more readily modify an existing prototype. The questionnaire responses from this study do not support these observations; indicating that the majority of participants were comfortable sketching and prototyping to share their ideas with others. However, participants were asked if they were comfortable modifying prototypes made by others in order to share ideas there was far greater variance in responses, as illustrated by Figure 23.

Did you feel comfortable modifying other people's prototypes to share your ideas?

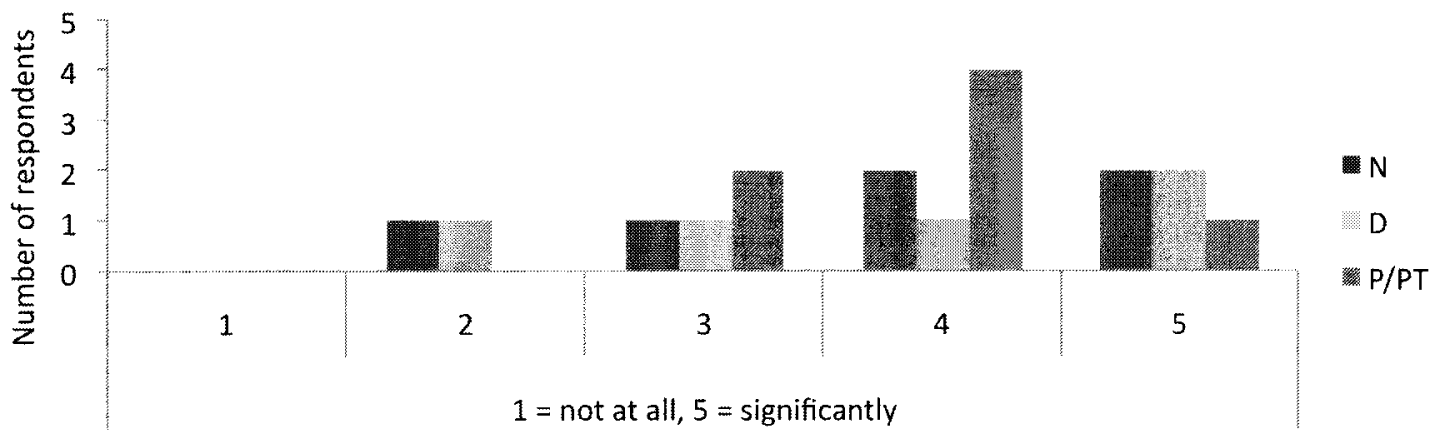

Figure 23. Feedback regarding the modification of prototypes to share ideas

One of the objectives of this study was to examine how sketches and prototypes support collaborative design development. To this end, a series of questions asked participants their perception of how these activities helped the group to (a) communicate, (b) develop shared understanding and (c) develop the final solution concepts. Both sketching and prototyping were perceived as helpful across all disciplines, as illustrated 
in Figure 24. This supports the use of these tools in early collaborative design development.

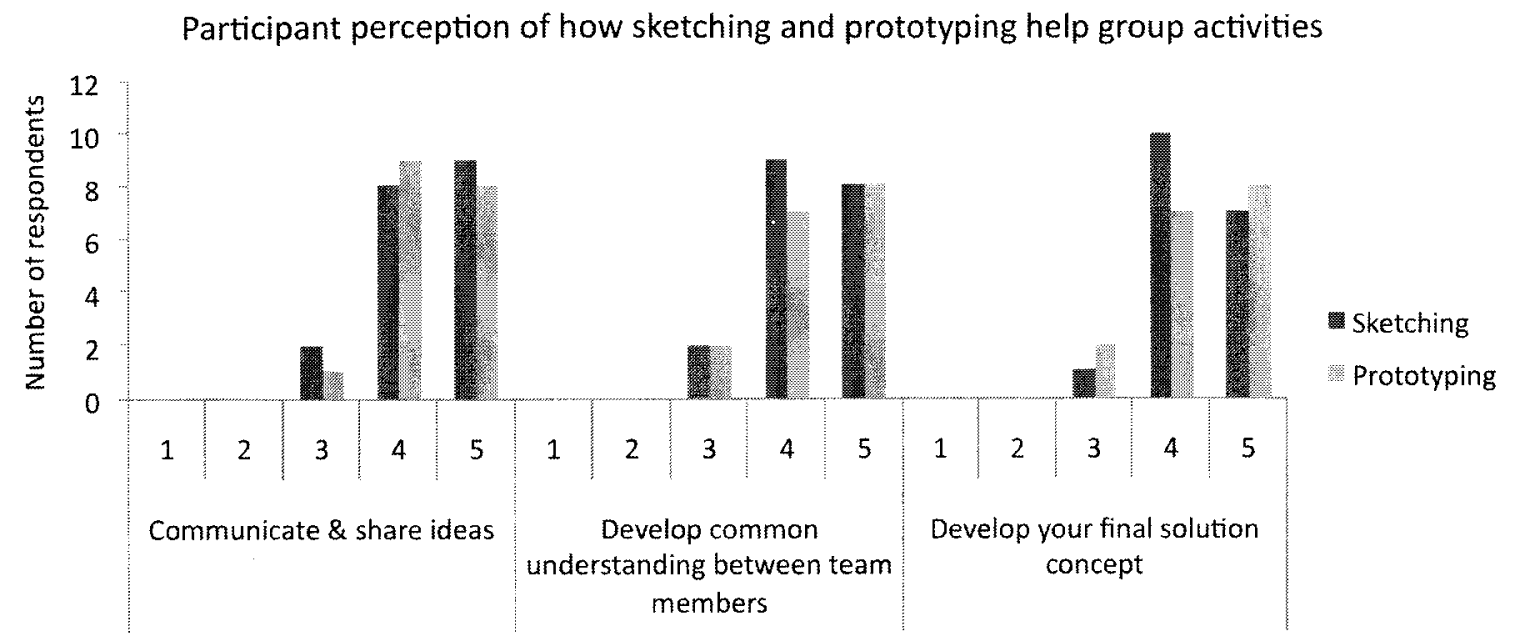

Figure 24. Participant perception of sketching and prototyping

\section{Feedback on group dynamics.}

When asked how the group self-organized, 13 participants indicated that everyone participated equally, six indicated that one person took a leadership role, and four did not respond. Of those where there was an emergent leader, responses were split between situations where the leader encouraged equal participation and encouraged participation, but also tried to push their own ideas forward. The demographic data collected in the survey does not allow for individuals to be identified and so there is no way to tie the responses to a specific group or individual. However, in all cases the indicated leader was a designer. One nurse participant commented "Designer had a great deal of ideas and moved things along quickly - a model would take me days".

Most comments were positive with respect to group dynamics and suggest that this influenced enthusiasm and performance in the small groups. For example: "People fed off of each other's ideas", "Everyone seemed to feel free to articulate ideas, move 
pieces of design around", "There was certainly a plenty of creative energy!" and "Great cross-mix of people/input".

\section{General impressions.}

Overall, feedback was very positive with $84 \%$ of respondents scoring their overall impression of the workshop as valuable or extremely valuable. Furthermore, $89 \%$ of respondents indicated they would encourage others to participate in a similar collaborative design activity. A limited number of hospital staff indicated neutrality with respect to one or both of these questions, assigning a score of three out of five. All three were participants in CS2. However, in general participating health care representatives stated the workshop was "great fun" and indicated the experience was "very beneficial for the hospital members". Furthermore there was positive feedback relating to the utility of this method in addressing existing challenges in the healthcare system. Respondents stated that this was an "Excellent session to move this project to a positive outcome", a "Fabulous idea - need to use this expertise for other workflow issues" and an "Excellent way to get people talking". In addition to questionnaire responses many participants also expressed positive feedback verbally either during or after the session: "To think, I went to school to be a nurse and now I'm designing a med room!"

Time was one of the variables considered during protocol development. As discussed in the methods section of this thesis, the original five-hour workshop proposed by Chung (2009) was shortened due to constraints imposed by the hospital environment. Informal observations during the sessions suggest that some participants were time conscious. One hospital staff member wrote "match time lines - session ran a little over the time allocated". However, feedback from other hospital staff suggests that after 
participating in the session the potential value of a longer session was recognized. "I

think if there would have been a bit more time we could've gotten more ideas out! But overall I enjoyed the session." It is not surprising that many of the designers agreed, given that the design process is part of their daily professional routine. One respondent stated "[This was a] great starter for getting physical \& visualizing for broader input ... this is a very complex issue being touched on in a bare minimum of time". Another wrote, "Very short time; would like more time for prototyping. Many further points (i.e. nurses having to bend over into drawers) came out while prototyping". A third participant expressed interest in continuing the process: "Would like to do a second session, after a short (two day) rest - more time in the session three to four hours".

\section{Summary of feedback from participants.}

In summary, hospital staff responded positively to the collaborative sessions, were open and enthusiastic regarding the CFCPing method, and saw opportunities to use this type of concept development strategy for other problems in the hospital context. The participating designers agreed that more time would have been beneficial, but also enjoyed the experience. Results from the questionnaire analysis suggest that Pictionary is not an ideal warm up exercise to encourage non-designers to participate more fully and without hesitation in sketching and prototyping activities. Finally, participants say they are comfortable sketching and prototyping to express and develop ideas, but less comfortable modifying prototypes. This is somewhat different than what was observed in small group activity where the majority of non-designers appeared to be more comfortable gesturing at sketches, than sketching themselves, and more comfortable modifying or suggesting modifications to prototypes than physically testing ideas. 
INTERACTIONS IN COLLABORATIVE PROTOTYPING

\section{Contribution}

This research explored interdisciplinary interaction during early concept development to gain a better understanding of how disciplinary roles and prototyping methods support collaboration in small groups. Results from the analysis of observed behaviour, outcomes from the collaborative sessions, and feedback from participants offer a number of contributions towards a better understanding of interdisciplinary interactions in CFCPing. First, this study reports examples where quick prototypes or pieces of an existing model were used by participants to support explanation, testing and generation of alternatives. This illustrates how prototyping can be used as a means to support concept development and confirms findings reported in initial CFCPing studies (Chung, 2009). Second, the comparison of verbal and physical behaviours across participating disciplines revealed that designers and non-designers assumed different roles and suggested that the facilitative role adopted by designers was beneficial in engaging non-designers in prototyping activities. Third, sequential analysis of interactions between participants revealed that more frequent transition between ideation and evaluation activities can help to generate more ideas. Together these findings suggest a future role for designers as consulting facilitators of collaborative design development and provide empirical data supporting the use of CFCPing in a healthcare context. Though this thesis focused on medication error in a pediatric surgical unit, thorough documentation of the research methods, analysis methods, and lessons learned from decisions regarding the preparation and conduct of the collaborative sessions could serve as a guide to future researchers using collaborative prototyping methods in other contexts. For example, CFCPing could be used to support the development of remote 
monitoring equipment, electronic health records and other tools that have multiple users with a variety of needs and sophisticated technological requirements. The implications of these contributions and opportunities for future research are discussed in the following section. 
INTERACTIONS IN COLLABORATIVE PROTOTYPING

\section{Discussion}

\section{Implications for CFCPing}

The outcomes of a number of decisions made during the preparation and conduct of the collaborative sessions in this study can be of insight to future organizers of CFCPing, specifically with respect to the selection of materials and the organization of participants during small group work. In addition, results suggest a new role for designers participating in CFCPing sessions.

\section{Preparation and Materials.}

One of the first decisions made in preparing for the collaborative sessions in this research was to shorten the full-length CFCPing session, outlined by Chung (2009, p. 118). This decision was based on feedback from hospital management that it would be very difficult to secure participants for a four or five-hour session. However, at the end of the sessions a number of participants commented that they would have liked more time to further develop solutions. This suggests that the full-length CFCPing schedule, including three iterations of ideation and concept development may actually be more feasible in this context than originally anticipated. More time would allow for added group discussion, critique and further development of design concepts. Second, the use of Pictionary as a warm-up exercise to encourage non-designers to participate in sketching and prototyping activities did not appear to be overly successful and observations suggested that non-designers may lack experience with creative brainstorming (Olson, 1963). Though the rules of creative brainstorming were highlighted in the introduction to the collaborative session, both by the lead facilitator 
and in the IDEO video clip used to introduce the design method, rejection, doubt and hasty evaluation of ideas was exhibited by hospital staff. In these early stages designers and other emergent leaders encouraged these doubts to be put aside in the spirit of brainstorming. This suggests that the rules of brainstorming need to be further emphasized and reinforced, especially during the first breakout into small groups, which points to a potential consulting facilitator role for designers in this type of activity. Finally, though in both sessions, participants were encouraged to generate 'wild ideas' and not be restricted to the current physical layout of the surgical unit, the design concepts generated during the first session were all within the envelope of the current space. Therefore the medication room and surgical unit floor plans provided during the first session were not provided to participants in the second session in an effort to encourage groups to 'think outside the box'. Concepts generated during the second session departed more from the rectangular layout and scale of the current design. Though not a specific focus of this study, this emphasizes need for careful selection of reference materials, keeping in mind the potential impact of these references on the design concepts generated by participants.

\section{Organization of Participants.}

Using the total number of verbal contributions as a measure of participation, it was revealed that in groups with more than four members and more than one representative from the same discipline, one participant contributed noticeably less than others. In addition, the five-person groups repeatedly self-organized into smaller groups of two and three, sometimes repeating and sometimes omitting information and explanation of ideas upon returning to the larger group. Some insight on the duplication 
of disciplinary expertise can be gained from comparing the results of this study to previous research on expert roles within groups. Specifically, the way members perceive or recognize the knowledge that is distributed amongst other members of the group affects the sharing of knowledge in that previously unshared information requires social validation before it will be accepted by the group (Argote, Gruenfeld, \& Naquin, 2001). This research states that social validation occurs when the accuracy of information provided by one participant is confirmed by another, but can also be provided if the speaker has recognized expertise or is assigned an expert role within the group. Based on this understanding, duplication of disciplinary representation may generate a requirement for information shared by one member to be confirmed by the second member from the same discipline, before being accepted and used by the group. In addition, recent research investigating relationships between the size and performance of small groups in organizations (Wheelan, 2009, p. 248) found that groups containing three to eight members were significantly more productive than groups with nine members or more, and groups containing three to four members were significantly more productive on a number of performance measures than groups with five to six members. In light of the aforementioned literature, the findings from this study suggest two implications for organizers of future CFCPing sessions: first, duplication of disciplinary representation within one small group may be less efficient in terms of developing shared knowledge within the group, and second that dividing into small groups of three or four, rather than five, may be more efficient during ideation and concept development. 


\section{A New Role for Designers in CFCPing.}

Observations from this research suggest there is a need for more encouragement for non-designer participants to engage in prototyping and maximize the potential benefits of this tool for knowledge management and concept development. Though all participants in this study indicated they were comfortable sketching and prototyping to share their ideas, non-designers typically gestured at reference material or at sketches produced by the designer in the group and engaged less frequently in sketching, writing and making activities. This type of hesitation was anticipated based on observations in initial CFCPing research conducted by Chung (2009) which led to the identification of two approaches to encouraging non-designer participation in CFCPing: (1) the use of

simple, flexible prototyping materials that are easy to manipulate, or (2) employing an assistant, skilled in prototyping and fabrication to create physical representations of ideas expressed by participants. This study employed the first option, based on the assumption that the participation of non-designers in the prototyping activity would facilitate the transfer, translation and transformation of knowledge between disciplines and promote engagement in concept development. This decision also acknowledged resource constraints present in the healthcare context that could make it difficult to engage prototyping assistants for each of the participating groups. Video analysis of small group activity in this study revealed that participation in visual representation by non-designers increased as the sessions progressed and with encouragement from participating designers. On occasion, participating designers intuitively played the role of model maker to facilitate the embodiment of ideas. There were other instances where designers lead by example, and once non-designers observed how easy it was to modify the simple 
materials, participation in sketching and prototyping increased. This suggests that other techniques not currently part of the CFCPing method could be used to increase nondesigner participation earlier in the collaborative prototyping session. Specifically, coaching, teaching and encouragement by designers appeared to be effective motivators of interdisciplinary participation. This leads to the question: could designers play a more facilitative role in CFCPing, encouraging the use of prototyping in collaborative activities? The identification of roles assumed by designers and non-designers during the collaborative session and characteristics of small group problem-solving processes defined through sequential analysis of interdisciplinary interactions also support this idea.

In this study, designers more frequently occupied a facilitative role, both with respect to the organization of group problem solving activities and by providing guidance and encouragement to non-designers engaged in sketching and prototyping. Conversely, non-designers provided more explanation and examples of the current context and design problems and were more likely to critique or reject ideas presented by others. As the collaborative concept development process reflects the design process and prototyping is also a design tool and therefore part of the disciplinary expertise contributed by designers, it is understandable that designers would demonstrate knowledge and expertise in these areas. However, the potential impact of the facilitative nature of this contribution warrants further investigation. The critiquing behaviour exhibited by non-designers is in direct conflict of the rules of creative brainstorming which were designed to support effective idea generation. Therefore, additional support for more visual representation of ideas and constructive concept development vs. rejection of early ideas could possibly 
lead to a more productive process. Encouraging this type of behaviour within a group could be a facilitative function.

The results from sequential analysis of interactions between participants also suggest possible benefits from facilitated interdisciplinary collaboration. In this study, groups that generated comparatively more ideas exhibited more frequent transitions between Ideation and Evaluation type exchanges and infrequent transitions between Information and Management type exchanges, compared to groups that spent a greater proportion of time planning and structuring the problem solving process. This suggests that frequent transitions between Ideation and Evaluation can lead to more effective collaboration. It also suggests that transitioning from Information to Management and spending a larger proportion of activity on process planning breaks a cycle of ideationinformation-evaluation. This can be related to the iterative cycle of ideationembodiment-critique articulated by Chung (2009), which has been identified as a key component of successful collaborative concept development. This understanding can also help define the nature of the facilitative role that could be formally adopted by designers in CFCPing.

\section{Implications for use of CFCPing in Healthcare Settings}

A primary contribution of this research is empirical evidence supporting the use of CFCPing in healthcare contexts. The interdisciplinary nature of health care and the complexity of problems faced by the current system point to the need for collaborative problem solving and development of innovative solutions, and this study illustrates that CFCPing is one method that can support the rapid development of solution concepts by interdisciplinary teams. 
The medication room design concepts generated during the CFCPing sessions highlight the preferences and priorities of nursing and pharmacy staff. They represent innovative ideas for workflow and space redesign and suggest the kinds of new technologies that would be most useful to clinical staff responsible for medication preparation and management on the pediatric surgical unit. These design concepts can be used to support the selection and development of new technologies and workflow design elements that will have the greatest positive impact on medication safety at the hospital.

Involving staff in the conceptualization and development of hospital and care improvement efforts is one way to foster a sense of ownership, and buy-in, which can also contribute to organizational change. In fact, interdisciplinary or interprofessional care has been identified as a means of addressing practice oriented and organizational challenges and as contributing to reducing the likelihood of patients experiencing adverse reactions (Canadian Health Services Research Foundation, 2006). Thus the positive response to CFCPing by nursing and pharmacy staff participating in this study suggests that CFCPing may be a useful tool that healthcare organizations can use to initiate employee involvement and support interprofessional teamwork. Furthermore, the sixth principle guiding the conduct of effective CFCPing sessions speaks directly to the objectives of interprofessional care: mutual learning is the engine for forward momentum in team collaboration.

Thus this research recognizes the potential for the use of design methods in Canadian hospitals to enable direct involvement in the development of user-centered solutions to complex patient safety issues, and organizational challenges. Specific opportunities for application of this method in healthcare facilities include: current 
challenges in the design of way finding or navigation systems for visitors; patient room design that serves both the needs of the patient and their family and supports safe, effective and efficient workflow for hospital staff; service design, and other process and workflow issues.

\section{Limitations of the Study}

This study has a number of limitations. First, it represents the conduct of two collaborative sessions addressing the problem of medication safety management in a pediatric hospital. Therefore the results of this study must be viewed in this context and should not be generalized or viewed as applying directly to a wider range of design problems and contexts. Second, three disciplines were represented in this study, but many interdisciplinary projects will require the participation of more than three disciplines. Therefore, the results presented in this study may not be generalized towards CFCPing sessions that involve more than three disciplines. Third, the product designers participating in the study all came from sole proprietor or small design businesses and all were originally trained at Carleton University School of Industrial Design. Therefore, it is conceivable that other designers from more diverse backgrounds might approach CFCPing differently. Furthermore, designers with significant experience working in the healthcare industry may have had a better initial understanding of the hospital environment and design challenge which could have impacted the problem solving process exhibited by small groups. It is difficult to predict the impact of these demographic characteristics on the design development process without conducting comparative studies. 


\section{Future Research}

This thesis represents one of the first empirical studies employing CFCPing. Further empirical research, testing the contribution of this method in a wider variety of settings, is required to allow for confirmation of the findings presented in this thesis and possible identification of other patterns in the behaviour and responses of participants. The most important recommendation for future research is to investigate the role of the designer as facilitator in CFCPing. Doing so may contribute to defining a future role for designers in interdisciplinary collaboration at the front end of design development.

Throughout this study, designers exhibited a number of behaviours (planning, ideas, coach/teach, making prototypes) that can be related to the definition of consulting facilitator provided by Schwartz et al (2005, p. 28). Essentially, consulting facilitators help a group increase its effectiveness by diagnosing and intervening on group process and structure, and also provide content expertise, in this case ideas. Ideation, prototyping and the design process are key activities in CFCPing and also professional skills possessed by designers. Therefore, designers may be ideally suited to provide additional support to the CFCPing process by acting as consulting facilitators within the small cross-functional teams characteristic of this method. However, facilitation is itself a skill (though some individuals may be naturally better than others) that requires training and experience (Schwartz, 2005). In addition, CFCPing is supposed to be spontaneous, providing as much opportunity as possible for the development of new and innovative ideas. So, though too much planning and direction may stunt the creative potential of a group, as illustrated by some of the findings in this study, careful facilitation may also help to fend off some of the negative factors affecting group performance and support the 
use of prototyping to the maximum potential, as illustrated by other findings in this study. Future research could investigate the impact of assigning a facilitative role to designer participants in CFCPing by:

1. Defining the consulting facilitator role for CFCPing activities;

2. Developing guidance material for designers assuming this role; and

3. Conducting empirical studies with a control group and test group.

In the suggested research, participating designers in the control group would be given no special direction, as in the research presented in this thesis. In the test group designers would be assigned a consulting facilitator role. Using the same design challenge across control and test groups would allow for a comparison of outcomes between groups and support the identification of differences in performance associated with the new role of designers as consulting facilitators. It should be noted that assigning the consulting facilitator role to designers participating in CFCPing sessions would not affect the leading facilitator role of the organizer of the session and only influence the behaviour of designers when working in small interdisciplinary teams to brainstorm solutions, prototype and develop design concepts.

This thesis represents an important precedent for future studies that focus on interactions in collaborative prototyping or collaborative initiatives in healthcare environments and also suggests an opportunity to investigate the role of designers as facilitators in cross-functional collaborative prototyping. 
INTERACTIONS IN COLLABORATIVE PROTOTYPING

\section{References}

ABC News. (1999, July 13). Deep Dive [ABC Nightline]. Retrieved from www.ABCNewstore.com.

Argote, L., Gruenfeld, D., \& Naquin, C. (2001). Group learning in organizations. In M. Turner (Ed.), Groups at Work: Theory and Research (pp. 369-411). London: Lawrence Erlbaum Associates.

Austin, S., Steele, J., MacMillan, S., Kirby, P., \& Spence, R. (2001). Mapping the conceptual design activity of interdisciplinary teams. Design Studies, 22(3), 211-232. doi: 10.1016/S0142-694X(00)00026-0.

Bakeman, R. \& Gottman, J. (1997). Observing interaction: An introduction to sequential analysis (2nd ed.). Cambridge, UK: Cambridge University Press.

Baker, G., Norton, P., Flintoft, V., Blais, R., Brown, A., Cox, J., .. Tamblyn, R. (2004). The Canadian Adverse Events Study: The incidence of adverse events among hospital patients in Canada. CMAJ, I70(11), doi: 10.1503/cmaj.1040498. Retrieved from http://www .cmaj.ca/cgi/content/full/170/1 1/1678.

Bodker, S., Ehn, P., Knudsen, J., Kyng, M., \& Madsen, K. (1988). Computer support for cooperative design. Proceedings from CSCW'88: Conference on Computer Supported Cooperative Work, 377-393. Portland, OR: ACM.

Bodker, S., \& Iversen, O. (2002). Staging a professional participatory design practice: Moving PD beyond the initial fascination of user involvement. Proceedings from NordiCHI'02: The second Nordic Conference on Human-computer Interaction. New York, NY: ACM.

Brodersen, C., Dindler, C., \& Iversen, O. (2008). Staging imaginative places for participatory prototyping. CoDesign, 4(1), 19-30. doi: 10.1080/15710880701875043. 
Campbell, C., Katzur, E., \& Wilhelm, D. (2008). Interdisciplinary design development: Why all the interest now? (unpublished research paper).

Campbell, C. (2009). Sketching for design development: An exploration of why and how visual representations support interdisciplinary design. (unpublished research paper).

Campbell, C., Parush, A., Ellis, J., Vaillancourt, R., Lockett, J., Lebreux, D., ... Pascuet, E. (2010a). A human factors approach for evaluating practices surrounding intravenous morphine and hydromorphone use in a pediatric hospital surgical unit [Poster]. Presented at Canada's Forum on Patient Safety and Quality Improvement, April 12-14, 2010.

Campbell, C., Parush, A., Ellis, J., Vaillancourt, R., Lockett, J., Lebreux, D., ... Pascuet, E. (2010b). A human factors approach to evaluating practices surrounding IV bolus morphine administration on a pediatric surgical unit [PowerPoint slides]. Presented at the International Clinical Nursing Research Conference 2010. Ottawa, ON.

Campbell, C., Parush, A., Ellis, J., Vaillancourt, R., Lockett, J., Lebreux, D., ... Pascuet, E. (2010c). Prioritizing Risk Factors Related to Morphine Administration in a Pediatric Surgical Unit [Poster]. To be presented at CAPHC'10: The Canadian Association of Paediatric Health Centers Annual Conference. Winnipeg, MB.

Cagan, J., \& Vogel, C. (2002). Creating breakthrough products: Innovation from product planning to program approval. Upper Saddle River, NJ: Prentice Hall PTR. Canadian Health Services Research Foundation. (2006). Teamwork in healthcare: Promoting effective teamwork in healthcare in Canada. Ottawa, ON: CHSRF. Retrieved from: www.chsrf.ca/research_themes/pdf/teamwork-synthesis-report_e.pdf.

Canadian Institute for Health Information. (2004). Health care in Canada. Statistics Canada. 
Canadian Institute for Health Information. (2007). Patient safety in Canada: An update.

Retrieved from: www.cihi.ca.

Canadian Patient Safety Institute. (2005). About CPSI. Retrieved from:

http://www.patientsafetyinstitute.ca/patientsafety/about.html

Carlile, P. (2002). A pragmatic view of knowledge and boundaries: Boundary objects in new product development. Organization Science, 13(4), 442-455.

Carlile, P. (2004). Transferring, translating, and transforming: An integrative framework for managing knowledge across boundaries. Organization Science, 15(5), 555-568. doi: $10.1287 /$ orsc. 1040.0094 .

Chung, W. (2008). Research interest: Interdisciplinary collaboration in product development [PowerPoint slides]. (unpublished).

Chung, W. (2009). Early collaborative prototyping - A lecture for IDES5103, Winter 2009 [PowerPoint slides]. (unpublished).

Chung, W. (2009). Cross-functional collaborative prototyping in the front end of the design process (Doctoral dissertation, Illinois Institute of Technology, 2009).

Committee on Facilitating Interdisciplinary Research, National Academy of Sciences, National Academy of Engineering, Institute of Medicine. (2004). Facilitating interdisciplinary research. Washington, DC: National Academies Press.

Curran, V. (2007). Primary health care transition fund: Collaborative care. Ottawa, ON: Health Canada. Retrieved from http://www.healthcanada.gc.ca/phctf.

Dahan, E., \& Hauser, J. (2002). Managing a dispersed product development process. In B. Weitz \& R. Wensley (Eds.), Handbook of Marketing (pp. 179-222). Sage Publications Ltd. 
Etchells, E., Juurlink, D., \& Levinson, W. (2008). Medication errors: The human factor [Commentary]. CMAJ, 178(1), 63-4.

Floyd, C. (1984). A systematic look at prototyping. In Budde, R. (Ed.), Approaches to prototyping: Proceedings of the working conference on prototyping. Berlin: Springer Verlag.

Fulton Suri, J. (2008, March). Informing our intuition: Design research for radical innovation. Rotman Magazine, 52-57.

Gregory, J. (2003). Scandinavian approaches to participatory design. International Journal of Engineering Education, 19(1),62-74.

Goldschmidt, G. (2007). To see eye to eye: The role of visual representations in building shared mental models in design teams. CoDesign, 3(1), 43-50. doi: $10.1080 / 15710880601170826$.

Gosbee, J. (2004). Introduction to human factors engineering series. Joint Commission Journal of Quality and Safety, 30(4), 215-220.

Hall, P. (2005). Interprofessional teamwork: Professional cultures as barriers. Palliative Medicine, 1, 188 - 196. doi: 10.1080/13561820500081745.

Health Force Ontario. (2007). Interprofessional care: A blueprint for action. Retrieved from: ht1p:/www healthforceontario.ca/upload/en/whatishfo/ipc\%20blueprint\%20final.pdf.

Hicks, R. (2006). A mixed-methods analysis of pediatric medication errors from the perioperative setting (Doctoral dissertation, Capella University, 2006).

Hoonhout, H. (2007). Setting the stage for developing innovative product concepts: People and climate. CoDesign, 3(1), 19-34. doi: 10.1080/15710880701376752. 
Howard-Grenville, J. \& Carlile, P. (2006). The incompatibility of knowledge regimes:

Consequences of the material world for cross-domain work. European Journal of

Information Systems, 15, 473-485.

IDEO. (n.d.). DIEGO powered dissector system for Gyrus ACMI, ENT Division: A benchmark in user-centered medical device design. Retrieved from:

http:/www.ideo.com/work/item/diego-powered-dissector-system/.

International Ergonomics Association. (2000). The discipline of ergonomics. Retrieved from:

http://www.iea.cc/browse.php?contID=what_is_ergonomics.

Industrial Design Society of America. (2009). ID defined. Retrieved from: www.idsa.org.

Isaksen, S. (1998). A review of brainstorming research: Six critical issues for inquiry. Creativity Research Unit - Monograph \#302. Buffalo, NY: Creative Problem Solving Group Buffalo. Retrieved from http://www.cpsb.com/resources/downloads/public/302Brainstorm.pdf.

James, K., Barlow, D., McArtney, R., Hiom, S., Roberts, D., \& Whittlesea, C. (2009). Incidence, type and causes of dispensing errors: A review of the literature. International Journal of Pharmacy Practice, 17(1), 9-10-30.

Kelly, T. (2001). Prototyping is the shorthand of innovation. Design Management Journal, 12(3), $35-42$.

Kim, Y., Kim, M., \& Wilde, D. (2008). Toward the management of design creativity. Design Management Journal, 3(2), 45-52. Retrieved from: www.dmi.org.

Kohn, L., Corrigan, J. and Donaldson, M. (Eds). (2000). To err is human: Building a safer health system. Washington, D.C: National Academy Press. 
Kramer, C. (2009). Communication linking team mental models and team situation awareness in the operating room (Master thesis, Carleton University, 2009).

Kucklick, T. (2006). The medical device $R \& D$ handbook. London: Taylor \& Francis.

Kumar, V. (2004). Innovation planning toolkit. Paper presented at FUTUREGROUND International Conference in Melbourne, November 17-21, 2004. Retrieved from www.id.iit.edu.

Lee, J., Popovic, V., Blackler, T., \& Lee, K. (2009). User-designer collaboration during the early stage of the design process. In Proceedings from IASDR 2009, (pp. 1-12). Retrieved from http://www.iasdr2009.org/m12.asp.

Leonard, D. (2007). Market research in product development. In K. Ichijo \& I. Nonaka (Eds.), Knowledge creation and management: New challenges for managers. USA: Oxford University Press. Retrieved from http//lib.myilibrary.com.proxy.library,carleton.ca.

Leonard, D., \& Swap, W. (1999). When sparks fly: Igniting creativity in groups. Boston: Harvard Business School Press. Retrieved from: NetLibrary.

Lewis, S., Mateas, M., Palmiter, S., \& Lynch, G. (1996). Ethnographic data collection and analysis for product development: Our experiences with a collaborative process. Interactions. doi: 10.1145/242485.242505.

Muller, M. (1992). Retrospective on a year of participatory design using the PICTIVE technique. Computer Human Interaction, 455-463. ACM.

Muller, M. (2003). Participatory design: The third space in HCI. In J. Jacko \& A. Sears (Eds.), The human-computer interaction handbook: Fundamentals, evolving technologies and emerging applications (pp. 1051-1068). Mahwah, NJ: Lawrence Erlbaum Associates, Inc. 
Nonaka, I. \& Takeuchi, H. (1995). The knowledge-creating company: How Japanese companies create the dynamics of innovation. New York, NY: Oxford University Press.

Olson, G., Olson, J., Carter, M., \& Storrosten, M. (1992). Small group design meetings: An analysis of collaboration. Human-Computer Interaction, 7(4), 347-374. doi: 10.1207/s15327051hci0704_1.

Osborn, A. (1963). Applied imagination (3rd ed.). New York: Charles Scribner's Sons.

Paulus, P., \& Nijstad, B. (2003). Group creativity: Innovation through collaboration. New York, NY: Oxford University Press.

Paulus, P. \& Brown, V. (2003). Enhancing ideational creativity in groups: lessons learned from research on brainstorming. In P. Paulus, \& B. Nijstad (Eds.), Group creativity: innovation through collaboration. New York, NY: Oxford University Press.

Parshuram, C., To, T., Seto, W., Trope, A., Koren, G., \& Laupacis, A. (2008). Systematic evaluation of errors occurring during the preparation of intravenous medication. Canadian Medical Association Journal, 178(1), 42-8. Retrieved from CBCA Reference database (Document ID: 1403865621).

Parush, A., Campbell, C., Ellis, J., Vaillancourtt, R., Lockett, J., Lebreux, D., ... Pascuet, E. (2010). A human factors approach to evaluating morphine administration in a pediatric surgical unit. In V. Duffy (Ed.), Advances in human factors and ergonomics in healthcare (pp. 468-477). CRC Press.

Philp, K. (2007). When is more heads better than one? Creativity, quantity and quality in idea generation (M.A. Thesis, Carleton University, 2007).

Robillard, P., D'Astous, P., Detienne, F., \& Visser, W. (1998). Measuring cognitive activities in software engineering. In Proceedings from The 20th International Conference on 
Software Engineering (pp. 292-300). Kyoto, Japan: IEEE Comput. Soc. doi:

\subsection{9/ICSE.1998.671342.}

Robillard, P., Astous, P., Detienne, F., \& Visser, W. (1998). An empirical method based on protocol analysis to analyze technical review meetings. In Proceedings from The 1998 conference of the Centre for Advanced Studies on Collaborative Research (pp. 1-12). Toronto, Canada: IBM Press.

Sanders, E. (2004). Ethnography and the empowerment of everyday people. Whitepaper for Microsoft Corporation. Retrieved from http://www.maketools.com/articlespapers/EthnographyandEmpowerment_Sanders_04.pdf.

Sanders, E. (2005). Information, inspiration and co-creation. In Proceedings from The 6th International Conference of the European Academy of Design. March 29-31, 2005. Bremen, Germany.

Sanders, E. (2006). Design research in 2006. Design Research Quarterly, 1(September), 1-2. Retrieved from www.designresearchsociety.org.

Sanders, E. \& Ivey, M. (2006). Designing a physical environment for co-experience and assessing participant use. In Proceedings from Wonderground: Design Research Society International Conference 2006. Lisbon.

Sanders, E., \& Stappers, P. (2008). Co-creation and the new landscapes of design. CoDesign, 4(1), 5-18. doi: 10.1080/15710880701875068.

Schön, D. (1983). The reflective practitioner. Cambridge, MA: MIT Press.

Schrage, M. (2000). Serious play: The future of prototyping and prototyping the future. Design Management Journal, 11(3), 50-57. 
Schwartz, R., Davidson, A., Carlson, P., \& McKinney, S. (2005). The skilled facilitator fieldbook: Tips, tools, and tested methods for consultants, facilitators, managers, trainers and coaches. San Francisco, CA: Jossey-Bass.

Sharek, P., McClead, R., Taketomo, C., Luria, J., Takata, G., Walti, B., ... Frederico, F. (2008). An intervention to decrease narcotic-related adverse drug events in children's hospitals. Pediatrics, 122(4), e861-6. doi: 10.1542/peds.2008-1011.

Sharp, H., Rogers, Y., \& Preece, J. (2007). Interaction design: beyond human-computer interaction $\left(2^{\text {nd }}\right.$ ed). Chichester: John Wiley \& Sons.

SonicRim. (2003). Tools in design research [PowerPoint presentation].

Smith, P. \& Reinertsen, D. (1991). Developing products in half the time. New York: Van Nostran Reinhold.

Star, S. \& Greisemer, J. (1989). Institutional ecology, 'translations' and boundary objects: Amateurs and professional in Berkeley's Museum of Vertebrate Zoology, 1907-1930. In Social Studies of Science, 19(3), p.387-420.

Sutton, R., \& Hargadon, A. (2010). Groups in brainstorming context: Effectiveness in a product design firm. Science, 4l(4), 685-718.

The Royal College of Physicians and Surgeons of Canada. (2003). Canadian patient safety dictionary. Ottawa, ON: Davies, J., Hébert, P., \& Hoffman, C.

Turner, M. (Ed.). (1999). Groups at work: Theory and research. Mahwah, NJ: Lawrence Erlbaum Associates Inc.

Turner, S. (2000). What are disciplines? In P. Weingart and N. Stehr (Eds.), Practicing interdisciplinarity (p. 46-65). Toronto, Buffalo, London: University of Toronto Press. 
Tversky, B. (2002). What do sketches say about thinking? In T. Stahovic, J. Landay, \& R. Davis (Eds.), Proceedings from AAAI spring symposium on sketch understanding. Pp. Menlo Park, CA: AAAI Press. Retrieved from http://wwwpsych.stanford.edu/ bt/diagrams/papers/.

Ulrich, K. \& Eppinger, S. (2000). Product design and development, (2 ${ }^{\text {nd }}$ ed). McGraw-Hill.

Wasson, C. (2000). Ethnography in the field of design. Human Organization, 59(4), 377-388.

Westerlund, B. (2007). A workshop method that involves users talking, doing and making. In Proceedings from the 7th International Conference on Human-Machine Interaction.

Wheelan, S. (2009). Group size, group development, and group productivity. Small Group Research, 40(2), 247-262. doi: 10.1177/1046496408328703. 


\section{Appendix 1. Collaborative Design Session Protocol}

\section{COLLABORATIVE DESIGN SESSION PROTOCOL}

This concept development session is part of the study $\mathbf{A}$ human factors approach for evaluating practices surrounding intravenous morphine or hydromorphone use in a pediatric hospital surgical ward. This project is being conducted in collaboration with CHEO and Carleton University.

\section{Purpose of the sessions}

The primary purpose of the sessions is to develop innovative solution concepts that address identified opportunities for improvement in IV bolus morphine and hydromorphone delivery. The resulting designs will provide the basis for recommendations for equipment, procedures and practices that will improve medication safety management at the hospital.

Data collected from these sessions will also support the research student's Master thesis in Design: investigating the role of designers in interdisciplinary collaboration at the front end of design development.

\section{Scope}

The focus of the session will be to re-design the medication management system on the surgical ward, including the preparation area and narcotic cabinet. A set of parameters and requirements will be provided (based on observation and interview data collected in earlier phases of this study) to support participants in the development of concepts that address identified user needs and critical factors affecting medication safety.

\section{Method}

This is a collaborative design session. Participants will be organized into interdisciplinary teams and asked to share knowledge and experience, using sketches and rough prototypes or mock-ups to represent their ideas.

\section{Participants}

Hospital staff and industry professionals with expert knowledge related to the identified design problem, including: pharmacy, pharmacy technicians, surgical ward nursing staff, nursing management, and industrial design professionals.

\section{Inclusion criteria}

Participants employed by the hospital should be currently working on the surgical ward, have a professional interest in pain management or patient safety and be knowledgeable about IV bolus morphine delivery, the pharmaceutical characteristics of the drug, and/or some aspects of the existing preparation and delivery process in the context of pediatric care. Diversity in the experience of participants is desirable as it provides different perspectives (e.g. senior and junior nursing staff, staff with experience working in other units/hospitals, teaching staff). 
Designers recruited to participate in the study will be professional product designers with a minimum of 5 years of work experience (this could be a combination of post-graduate work and industry experience). They must be sign any confidentiality agreements required by $\mathrm{CHEO}$ prior to the session.

\section{Facilities and materials}

The collaborative sessions will be conducted in one of the CHEO meeting rooms, with enough space to accommodate 12 participants together and also space to break into four small teams. Sessions will be conducted between $3: 30$ and 6:00pm on a weekday.

\section{Compensation}

Participants will be compensated at an average rate of $\$ 40 /$ hour. Participants that are staff at $\mathrm{CHEO}$ will receive their normal hourly wage and funding will be provided directly to managers so that additional staff may be scheduled to cover the work of those participating in the study. Industrial design participants will be compensated individually at the average rate. The total budget for the study remains unchanged and is within the funding committed through the MITACS ACCELERATE grant secured for this study.

\section{Conduct}

The session will start with an introduction of participants, a review of the existing workspace design, the identified and prioritized risk-factors, possible technology solutions and systems implemented at other hospitals.

Participants will be divided into four interdisciplinary teams.

Participants will be asked to sketch and prototype their ideas to support the externalization of tacit knowledge and development of solution concepts within their small teams.

The first collaborative session will be scheduled as soon as possible once REB approval has been secured. Additional sessions will be scheduled, based on availability of participants, to a maximum of three sessions. If there is insufficient number of participants in any one session, the number of breakout teams formed within the session may be reduced to two or three.

\begin{tabular}{lll}
\hline Time & Activity & Actors \\
\hline 15-30 min & $\begin{array}{l}\text { Introduction: present objectives, method and } \\
\text { schedule of activities. Divide participants into } \\
\text { small teams. }\end{array}$ & $\begin{array}{l}\text { Lead facilitator } \\
\text { (Campbell) }\end{array}$ \\
\hline 45-60 min & $\begin{array}{l}\text { Brainstorming and preliminary concept } \\
\text { development resulting in a series of small- } \\
\text { scale mock-ups }\end{array}$ & Participants \\
\cline { 2 - 3 } & $\begin{array}{l}\text { Presentation of concepts to the large group, } \\
\text { critiquing and improving design concepts }\end{array}$ & Participants \\
\hline
\end{tabular}




\begin{tabular}{lll}
\hline 45-60 min & $\begin{array}{l}\text { Design development \& refined direction } \\
\text { resulting in one small scale prototype } \\
\text { (meal/snack-time included) }\end{array}$ & Participants \\
\cline { 2 - 3 } & $\begin{array}{l}\text { Final prototype presentation using the } \\
\text { prototype as a basis for demonstration \& } \\
\text { discussion }\end{array}$ & Participants \\
\hline $15 \mathrm{~min}$ & $\begin{array}{l}\text { Debrief \& feedback from participants } \\
\text { Thank you to participants (compensation } \\
\text { packages) }\end{array}$ & All \\
\hline
\end{tabular}

\section{Feedback survey}

At the end of the session, participants will be asked to complete an anonymous feedback questionnaire soliciting their perception on the utility of the collaborative session and methods used. The questionnaire will be piloted and revised as appropriate.

\section{Sample feedback questions}

1. Please rate your impression of this workshop on a scale of 1 to $5(1=$ waste of time, $5=$ extremely valuable $) \quad 1-2-3-4-5$

Please use the space provided to explain your response

2. Would you encourage others to participate in a similar

$$
1-2-3-4-5
$$
collaborative design workshop? $(1=$ never, $5=$ absolutely $)$

3. Do you think the activity of sketching, to share your ideas, $1-2-3-4-5$ helped to develop a common understanding within your team?

$(1=$ not at all, $5=$ significantly $)$

4. Do you think using prototypes to share your ideas helped $1-2-3-4-5$ to develop a common understanding within your team? $(1=$ not at all, $5=$ significantly $)$

5. Your honest feedback is very much appreciated. Please use the space provided to add your recommendations for the session or additional comments for the study team. 


\section{Data collection \& analysis}

All sessions will be video and audio recorded to support data collection and analysis. Data will be abstracted to ensure anonymity. Recordings will be kept in the locked office of the study research assistant, who has signed a confidentiality agreement with CHEO. After the study is complete all computer files and video recordings will be stored in a locked cabinet and office in the Department of Psychology at Carleton University with access restricted to research staff who have signed confidentiality agreements with CHEO and for a period of 5 years before being permanently destroyed.

Effectiveness of the sessions will be evaluated based on the extent to which the final design concepts are developed in terms of form, function and satisfaction of the design criteria, which will be based on the needs identified through the observations and interviews described in the case study.

Recommendations developed through the sessions will be collected and evaluated as to their ability to address/mitigate factors that could potentially introduce error into the IV morphine delivery process.

Results will support 1) validation of data collected through observation and interviews, and 2) the development of recommendations to improve IV bolus morphine delivery at the hospital.

In addition, video recordings will be used to evaluate the sessions and collect data on the use of visual representations and knowledge sharing between disciplines during brainstorming and concept development. This analysis will be used to support the research student's Master thesis in Design.

\section{Publication of study results}

Final results of this study will be presented to the Director of Pharmacy in a final report and disseminated to CHEO management and staff as appropriate, including a lunch-andlearn for nursing staff, and presentation to the Nurse Advisory Council. Results may also be published in scientific theses, journals and presentations in aggregate so no subject can be identified. 


\section{Informed Consent for Focus Group Participants}

Title of Research Project: A human factors approach for evaluating practices surrounding intravenous morphine or hydromorphone use in a pediatric hospital surgical ward

Principal Investigator: $\quad$ Dr. Régis Vaillancourt, Director of Pharmacy, CHEO 613-737-7600 ext. 2231 / rvaillancourt@cheo.on.ca

Co-investigators: Dr. Jacqueline Ellis, Research Mentor/Nursing, CHEO

613-737-7600 ext.4140 / jellis@cheo.on.ca

Dr. Avi Parush, Academic Supervisor, Carleton University

613-520-2600 ext.6026/avi_parush@carleton.ca

Background: This study is being conducted in collaboration with Carleton University. Using a human factors perspective, it aims to uncover the contributing factors to morphine or HYDROmorphone medication errors in a post-surgery unit at the children's hospital. Study activities have included comprehensive observation of the entire medication process and capture of events and associated contextual factors that could potentially impact IV bolus morphine/HYDROmorphone delivery. Interviews were conducted to validate the observation results and obtain staff perception of existing supports and barriers to the safe delivery of IV bolus morphine/hydromorphone. The types of events, the association to other factors, and the comments made by nursing and medical personnel have been analyzed to identify and prioritize points in the process that could potentially contribute to medication error. The next step is to develop solutions and recommendations that will address the identified opportunities for improvement. This will be done through collaborative sessions with nursing staff and participants from other relevant disciplines.

Purpose of the focus groups: The purpose of these sessions is to support the development of innovative solution concepts that address identified opportunities for improvement in IV bolus morphine and hydromorphone delivery. The resulting designs will provide the basis for recommendations for equipment, procedures and practice that will improve medication safety management at the hospital.

Involvement: You are hereby invited to participate in a focus group session to be held on $[\mathrm{dd} / \mathrm{mm} / \mathrm{yyy}]$ at $\mathrm{CHEO}$ in room - The session will take approximately 2.5 hours to complete. During this time, participants will be asked to work in small interdisciplinary groups to brainstorm and develop solution concepts addressing factors affecting patient safety and the IV bolus morphine/hyrdromorphone preparation and delivery. Groups will be asked to sketch and build prototypes representing their ideas. Though some participants may be more experienced at drawing and making than others, everyone is encouraged to take part. Visually representing ideas, regardless of the quality of the sketches/prototypes, will help communication and concept development 
within the group. All required materials will be provided. Refreshments will be provided. The session will be video recorded for data collection and analysis purposes.

Compensation: Hospital staff will be compensated at their regular hourly rate. Designers will receive comparative financial and in-kind compensation.

Your participation is voluntary: Your participation is entirely voluntary and not workrelated. Your decision to participate (or not) in the research will in no way be reflection of your work. The decision to participate (or not) will not be shared with others. You are free to withdraw from the study at any time.

Potential Benefit: Your participation may lead to an increase in patient safety as a result of improvements to morphine or hydromorphone preparation, administration and nursing work-life based on the results of this study.

Who will be enrolled: Invited participants include key personnel involved in the medication process: staff that work on the Surgical Unit (5-East) and administer morphine and/or hydromorphone, nursing management, pharmacists and pharmacy technicians. Other participants will include professional product designers who bring expertise in product and systems design that may contribute to the development of solution concepts. Facilitators will include the research student (Catherine Campbell), design researchers, and human factors professionals from Carleton University.

Risks: There are no risks to you for your participation.

Withdrawal from study: You are free to withdraw from the study at any time without penalty. You can withdraw without giving any reasons for your decision.

Confidentiality: Your personal information will be kept strictly confidential except as required or permitted by law. You will not be personally identified in any publication or presentation of this study. All participants who are not employees of the hospital must sign the attached confidentiality agreement in addition to this informed consent form. Any personal information about you that leaves the hospital will be abstracted so that you cannot be identified by name. Only the principal investigators and the people conducting the focus groups will have access to names of participants. All computer files will be kept locked with restricted access passwords known only to the investigators. Lists of participants and video recordings will be kept in the locked office of the study research assistant, who has signed a confidentiality agreement with CHEO. After the study is complete all computer files and video recordings will be stored in a locked cabinet and office in the Department of Psychology at Carleton University, with access restricted to research staff who have signed confidentiality agreements with CHEO, for a period of 5 years before being permanently destroyed. Final results of this study will be published in scientific theses, journals or presentations in aggregate so no subject can be identified. If you are interested, you can request a copy of the study results at the end of the study. 
Obtaining Additional Information: The study is under the direction of Régis

Vaillancourt, Director of Pharmacy and the research student is Catherine Campbell (ccampbell@cheo.on.ca). You are encouraged to ask questions at any time during the study. In the event that you have unanswered questions about the study please call Régis Vaillancourt, Director of Pharmacy at 613-737-7600 x2231.

This study has been reviewed and received CHEO Research Ethics Board (REB) approval. The CHEO REB is a committee of the hospital that includes individuals from different professional backgrounds. The board reviews all research that takes place at the hospital. Its goal is to ensure the protection of the rights and welfare of people participating in research. The Board's work is not intended to replace the employee's judgment about what decisions and choices are best for them. You may contact the Chair of the Research Ethics Board for information regarding employee's rights in research studies at 613-737-7600 ext 3272.

\section{Consent:}

I am satisfied that the information contained in this consent form was explained to me, with the extent that I am able to understand it, and that all questions have been answered. I hereby give my consent to participate in this study and will sign this consent form. I acknowledge having received a copy of this consent form. I have read the above information and have had the opportunity to have my questions answered.

Witness (name)

Witness (signature)

Date $(d d / m m / y y y y)$
Participant (name)

Participant (signature)

Date $(d d / m m / y y y y)$ 
INTERACTIONS IN COLLABORATIVE PROTOTYPING

\section{Appendix 2. Collaborative Session Feedback Survey}

\section{INTRODUCTION}

This anonymous questionnaire is designed to solicit participant's perception on the utility of the collaborative session and appropriateness of methods used. Please answer the questions honestly. This will take no more than 10 minutes to complete. Your feedback is appreciated and will help to improve future sessions.

\section{DEMOGRAPHICS}

(Please circle the most appropriate response)

1. What is your discipline / area of expertise?
a. Design
b. Nursing
c. Pharmacist
d. Pharmacy technician
e. Other

\section{METHODS}

(Please circle the most appropriate number on the scale provided for each question).

2. Were the objectives of this collaborative activity clearly articulated $1-2-3-4-5$ at the beginning of the session? $(1=$ not clear at all, $5=$ very clearly)

3. Was the video clip a good introduction to the collaborative $1-2-3-4-5$ prototyping method? $(1=$ not at all, $5=$ significantly $)$

4. Did watching the video clip motivate you to participate in the $1-2-3-4-5$ collaborative activities? $(1=$ not at all, $5=$ significantly $)$

5. Was the Pictionary game a good warm up to sketching and $1-2-3-4-5$ prototyping with your team? $(1=$ not at all, $5=$ significantly $)$

6. Did you feel comfortable sketching to share your ideas within your $1-2-3-4-5$ group? $(1=$ not at all, $5=$ significantly $)$

7. Did you feel comfortable prototyping to share and develop your ideas? $(1=$ not at all, $5=$ significantly $)$

8. Did you feel comfortable modifying other people's prototypes to $1-2-3-4-5$ share your ideas? $(1=$ not at all, $5=$ significantly $)$

9. Please rate your perception of how the activity of sketching helped your group to:

a. Communicate and share ideas?

$$
(1=\text { not at all, } 5=\text { significantly })
$$


b. Develop common understanding between team
members?

$$
1-2-3-4-5
$$

$(1=$ not at all, $5=$ significantly $)$

$1-2-3-4-5$

c. Develop your final solution concepts?

$(1=$ not at all, $5=$ significantly)

10. Please rate your perception of how the activity of

prototyping/making mock-ups helped your group to:

a. Communicate and share ideas? $1-2-3-4-5$

$(1=$ not at all, $5=$ significantly $)$

b. Develop common understanding between team

$1-2-3-4-5$

members?

$(1=$ not at all, $5=$ significantly $)$

$1-2-3-4-5$

c. Develop your final solution concepts?

$(1=$ not at all, $5=$ significantly $)$

11. In your opinion, how closely did the outcomes of the collaborative session meet the objectives that were agreed to at the beginning of $1-2-3-4-5$ session? ( 1 = not at all, 5 = exactly)

If not, please use the space provided to briefly explain why

GROUP DYNAMICS

(Please circle the most appropriate response)

12. How did your group self-organize?

a. Everyone participated equally

b. One person took a leadership role

13. Do you think this affected how the group worked together?
a. Yes
b. No

If yes, how?

14. If there was a leader in your group, from what discipline was this individual?
a. Design
b. Nursing
c. Pharmacist
d. Pharmacy technician
e. Other

15. If there was a leader in the group, did this person

a. Encourage equal participation by all team members

b. Try to influence the group by pushing their own ideas forward,

c. Both at different times 
d. Neither, please explain

\section{GENERAL IMPRESSIONS}

(Please circle the most appropriate number on the scale provided for each question).

16. Please rate your overall impression of this workshop on a $1-2-3-4-5$ scale of 1 to $5(1=$ waste of time, $5=$ extremely valuable $)$

17. Would you encourage others to participate in a similar collaborative $1-2-3-4-5$ design workshop? $(1$ = never, 5 = absolutely $)$

18. Please rate your perception of the lead facilitator's performance in the following three areas:

a. Professional conduct $1-2-3-4-5$

$(1=$ terrible, 5 = outstanding $)$

b. Clarity of instructions $1-2-3-4-5$ $(1=$ terrible, $5=$ outstanding $)$

c. Organized $1-2-3-4-5$ $(1=$ terrible, $5=$ outstanding $)$

19. Use the space provided to provide additional comments and/or recommendations for improving the session. Your honest feedback is much appreciated. 
Appendix 3. Collaborative Session Introductory Presentation
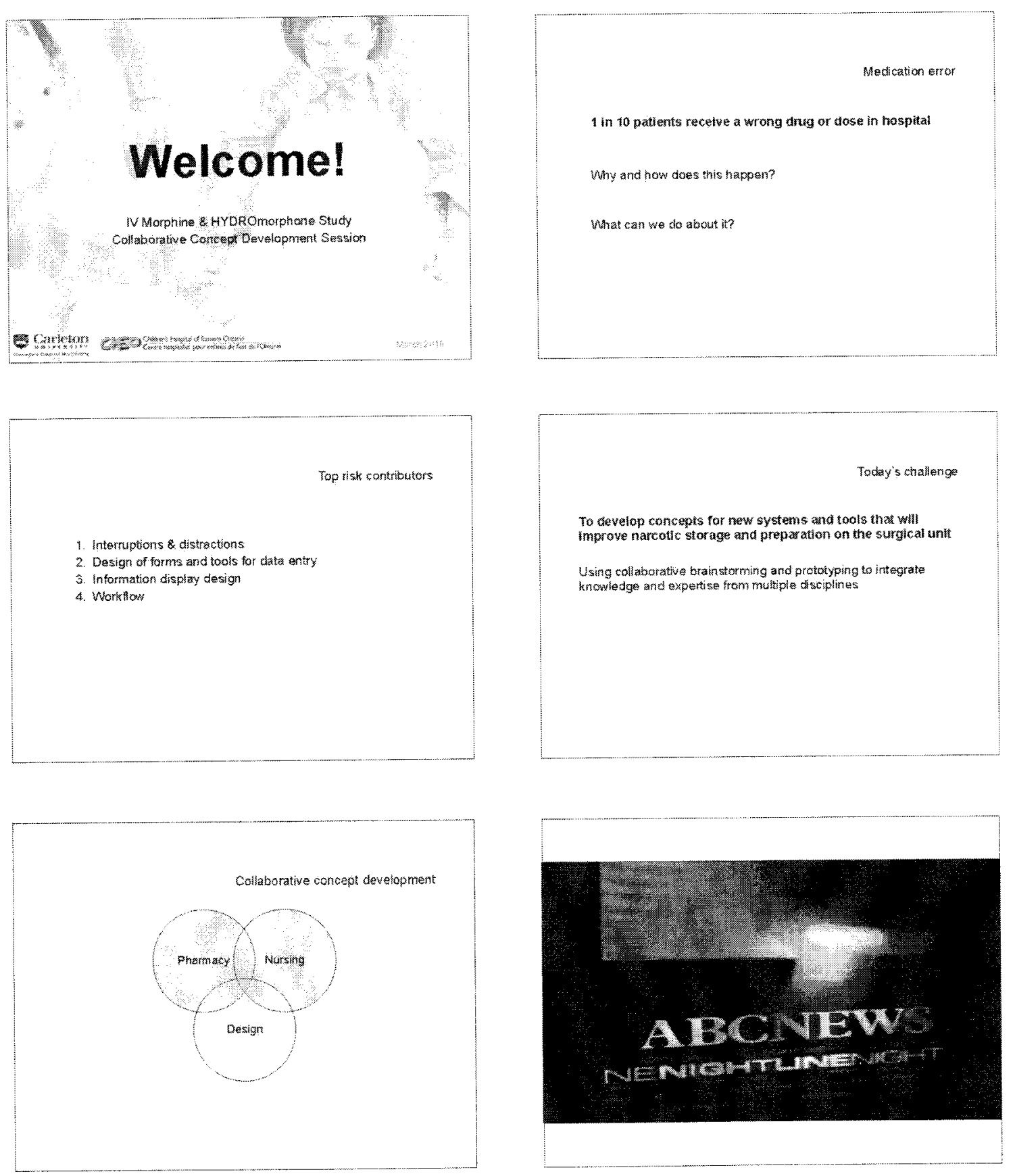

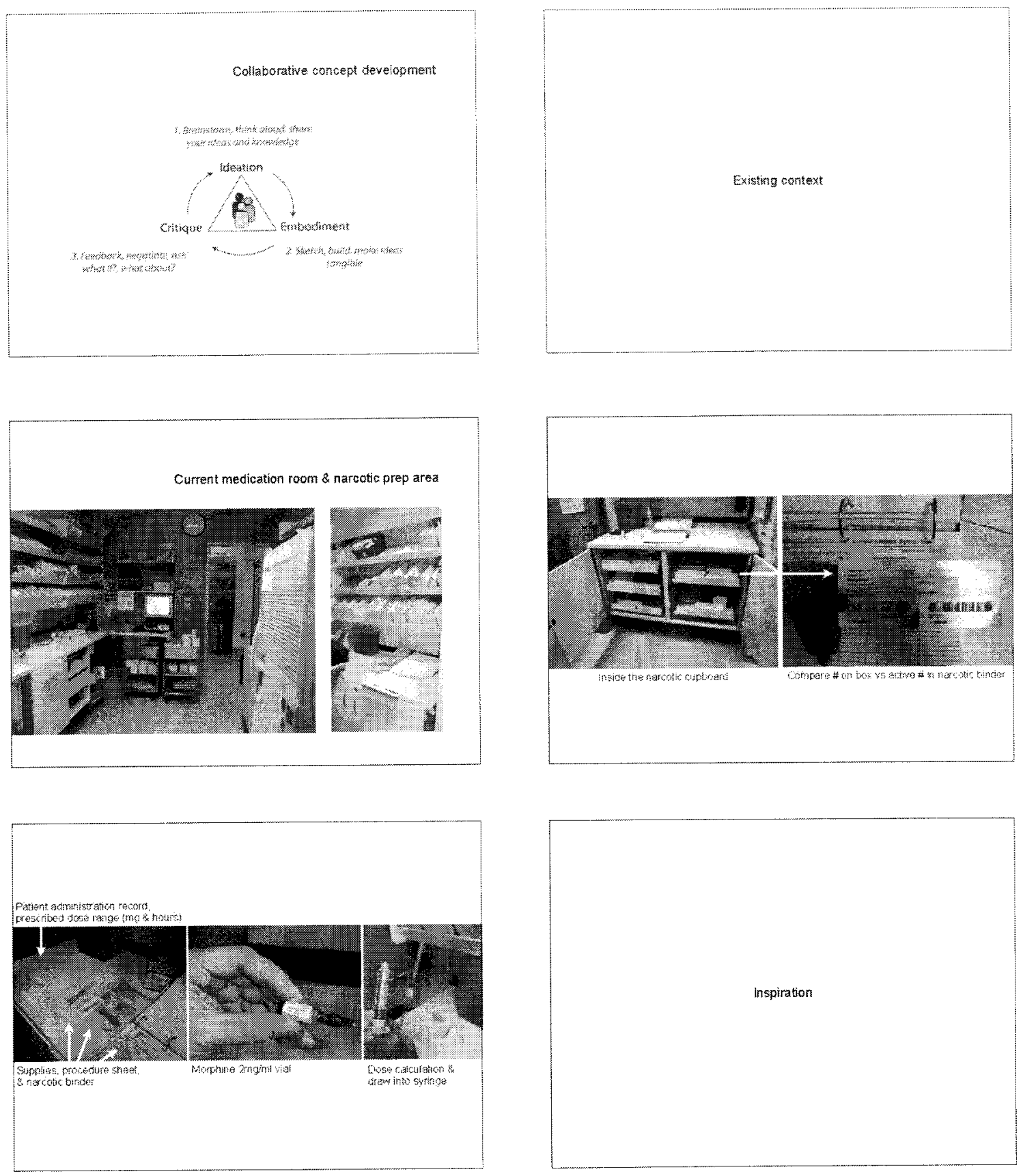

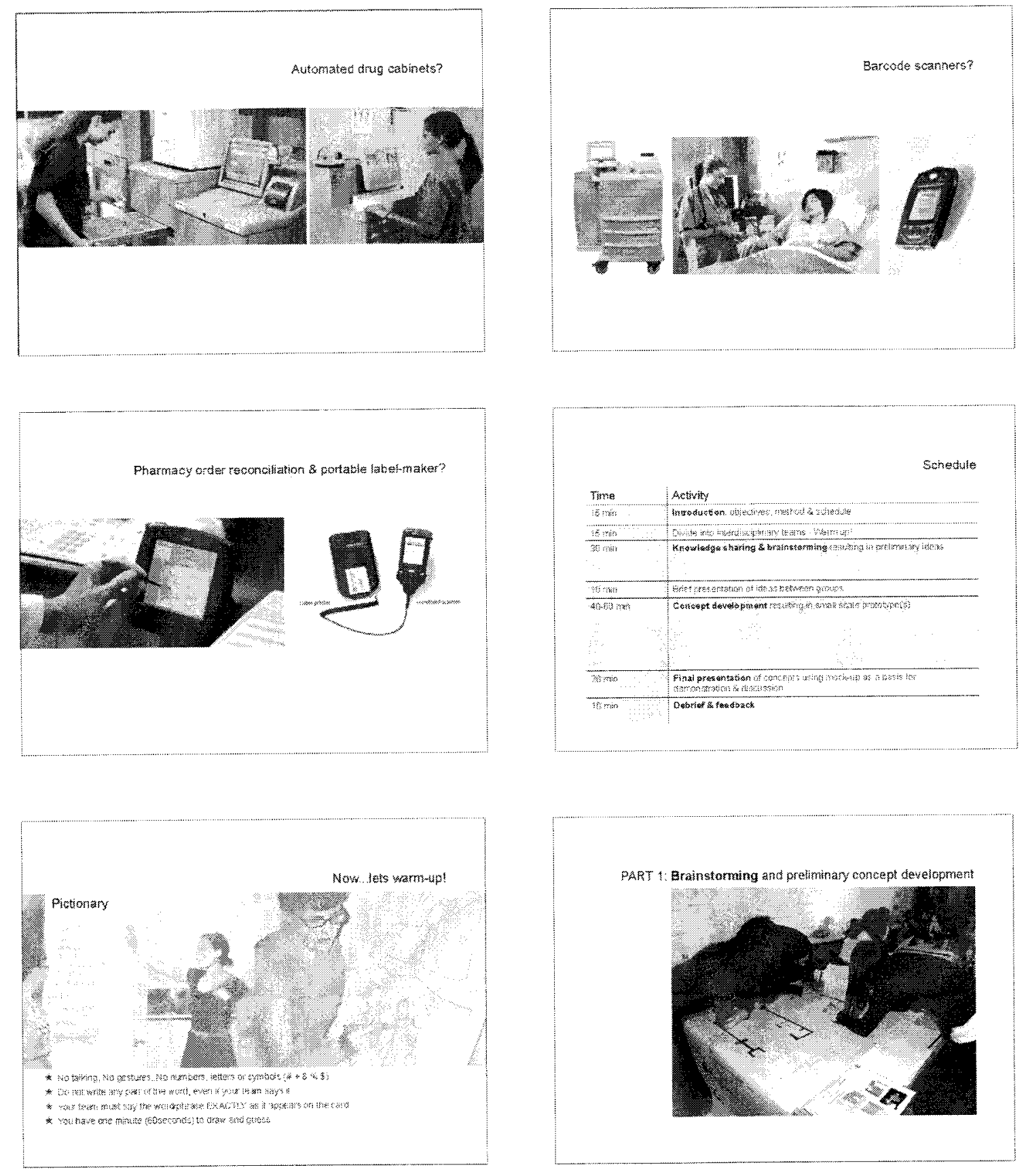

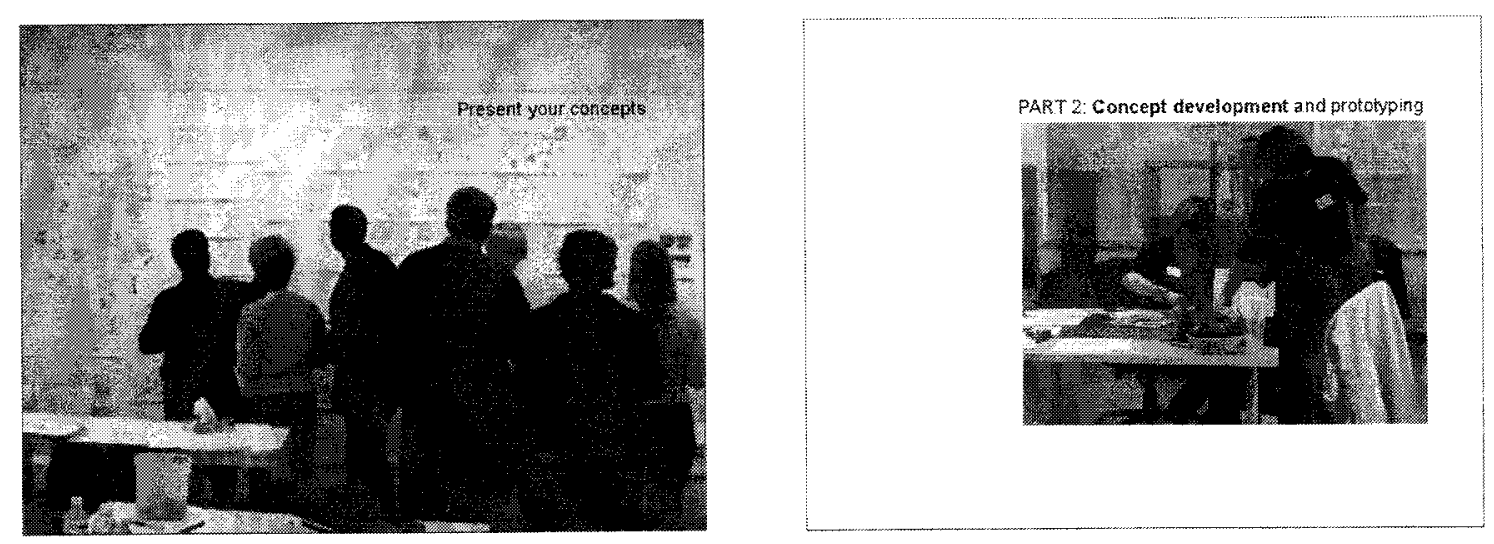


\section{Appendix 4. Behaviour Analysis by Discipline for Individual Groups}

Table 10 .

Example of preliminary interaction analysis for collaborative session \#1

\begin{tabular}{|c|c|c|c|c|c|}
\hline \multicolumn{6}{|c|}{ CS1-G3 (1) Individual Contributions } \\
\hline Verbal & Total & PT & $\mathrm{N}$ & $\mathrm{D}$ & Deseriptive analysis \\
\hline Question & 16 & 4 & 2 & 10 & $\begin{array}{l}\text { Designer asks most questions to Nurse and PT about current } \\
\text { context or design activities }\end{array}$ \\
\hline $\begin{array}{l}\text { Request } \\
\text { confirmation }\end{array}$ & 14 & 2 & 2 & 10 & Related to current context, problem, idea \\
\hline Statement & 36 & & & & Mostly related to current context and problem \\
\hline Suggest & 10 & 2 & 0 & 8 & $\begin{array}{l}\text { Designer starts by making suggestions to N regarding how the } \\
\text { group proceeds - planning ( } 3 \text { ), then offers solution ideas ( } 5 \text { ) } \\
\text { and then PT presents some ideas ( } 2 \text { ) }\end{array}$ \\
\hline Explain & 18 & 4 & 2 & 12 & $\begin{array}{l}\text { Designer does most of the explaining related to current } \\
\text { context, often highlighting problems }\end{array}$ \\
\hline Clarify & 8 & 4 & 4 & 0 & All cases are clarification of current context \\
\hline Relate to & 6 & 2 & 1 & 3 & Related to familiar concept and current context \\
\hline Agrees & 17 & 2 & 11 & 3 & Agreement on plan, current context and ideas \\
\hline Reject & 1 & & & & Rejects a problem raised by D \\
\hline Doubt & 2 & 2 & 0 & 0 & Both PT, idea and current context \\
\hline Evaluate & 4 & 2 & 2 & 0 & $\begin{array}{l}\mathrm{N} \text { related to planning suggested by } \mathrm{D} \text { and PT are current } \\
\text { context and idea }\end{array}$ \\
\hline Justify & 4 & 1 & 2 & 1 & Instances are spread amongst the group \\
\hline Coach/teach & 1 & 0 & 0 & 1 & $\begin{array}{l}\text { Designer is the source of the majority of process management } \\
\text { activities (coach, order, summary) }\end{array}$ \\
\hline Order & 1 & 0 & 0 & 1 & $\begin{array}{l}\mathrm{D} \text { asks } \mathrm{N} \text { to indicate busy areas on a floor plan of the current } \\
\text { unit }\end{array}$ \\
\hline Summary & 5 & 1 & 0 & 4 & Used by $\mathrm{D}$ to direct conversation \\
\hline Physical & Total & PT & $\mathrm{N}$ & $\mathrm{D}$ & 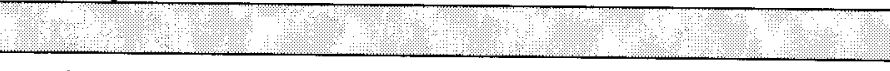 \\
\hline Make/modify & $\mathbf{0}$ & 0 & 0 & 0 & No instances of make in this initial brainstorming session \\
\hline Draw/sketch & 10 & 1 & 1 & 8 & All three sketch \\
\hline Gesture & 25 & 12 & 7 & 7 & $\begin{array}{l}\text { Gesture (referencing handout floor plan mostly) is dominated } \\
\text { by PT, but also used by N \& D to support development of } \\
\text { shared understanding }\end{array}$ \\
\hline Write on & 9 & 0 & 4 & 5 & $\begin{array}{l}\mathrm{N} \text { and } \mathrm{D} \text { do all of the writing, } \mathrm{N} \text { mostly because } \mathrm{D} \text { asked her to } \\
\text { indicate busy areas on the handout floor plan }\end{array}$ \\
\hline
\end{tabular}

Note: This represents the first 18 minutes of small group interaction for one group. 


\section{INTERACTIONS IN COLLABORATIVE PROTOTYPING}

\section{Appendix 5. Examples of Coded Dialogue}

The following tables show examples of how individual verbal and physical behaviour and exchanges between participants were coded in this study. These examples have been extracted from the data sheets used for coding an analysis and designed to exhibit specific elements of identified individual and collaborative behaviour. Therefore some tables display fewer levels of coding than the complete data set.

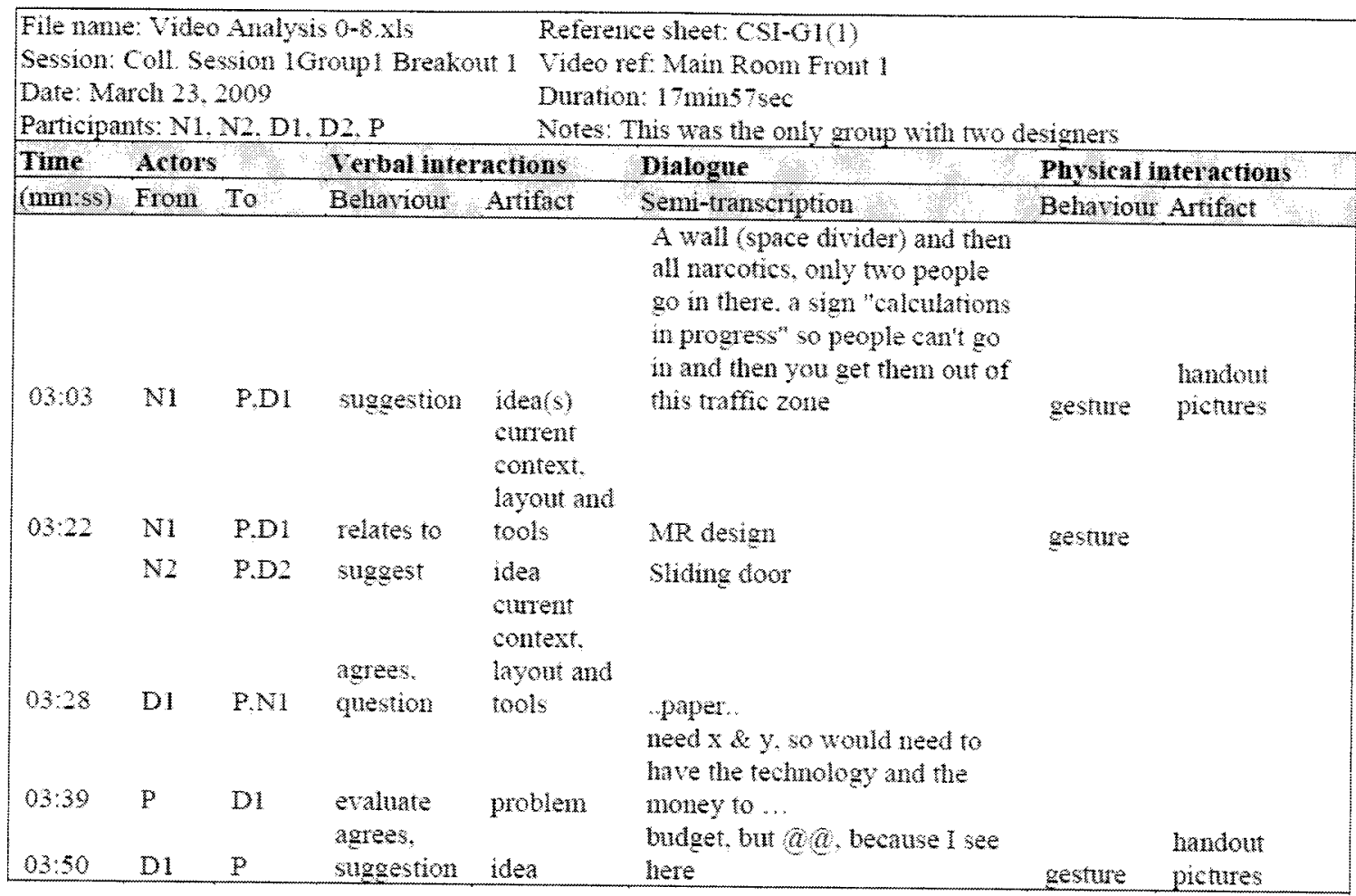

Figure 25. Example of a coded video clip 
Table 11.

\section{Example dialogue showing information exchange (verbal behaviour only)}

\begin{tabular}{|c|c|c|c|c|}
\hline Speaker & Receiver & $\begin{array}{l}\text { Verbal } \\
\text { behaviour }\end{array}$ & Artifact & Dialogue \\
\hline $\mathrm{D}$ & Team & question & problem & $\begin{array}{l}\text { So, without breaching your own confidentiality, when there is } \\
\text { an error...'cause I get the impression that it is not giving } \\
\text { morphine to the wrong kid, its usually too much for that } \\
\text { particular ... }\end{array}$ \\
\hline $\mathrm{P}$ & D & explain & $\begin{array}{l}\text { current } \\
\text { context } \\
\text { problem }\end{array}$ & $\begin{array}{l}\text { Sometimes when you have a teeny weeny baby, its so easy to } \\
\text { get mixed up between } 0.02,0.2 \text { and } 2 \text { and that's where we get } \\
\text { some of the } 10 \text { fold errors, and we've averted some of that by } \\
\text { going to the } 2 \mathrm{mg} / \mathrm{ml} \text { vials, but...such a wide assortments of } \\
\text { patient weights...there is no real standard dose you're using }\end{array}$ \\
\hline $\mathrm{N} 1, \mathrm{~N} 2$ & $P$ & agree & $\begin{array}{l}\text { current } \\
\text { context }\end{array}$ & hmm \\
\hline $\mathrm{N} 2$ & $\mathrm{P}, \mathrm{D}$ & $\begin{array}{l}\text { agree, } \\
\text { explain }\end{array}$ & $\begin{array}{l}\text { current } \\
\text { context }\end{array}$ & $\begin{array}{l}\text { That's right, because its all by weight, once you're an adult } \\
\text { and you're } 18 \text { or older we don't really go by weight anymore }\end{array}$ \\
\hline N1 & $\mathrm{D}$ & clarify & $\begin{array}{l}\text { current } \\
\text { context }\end{array}$ & $\begin{array}{l}\text { If you're over } 50 \mathrm{~kg} \text { then you sort of go into this generalized } \\
\text { category }\end{array}$ \\
\hline
\end{tabular}

Table 12.

Example dialogue showing information exchange (verbal \& physical)

\begin{tabular}{|c|c|c|c|c|c|c|}
\hline Speaker & Receiver & $\begin{array}{l}\text { Verbal } \\
\text { behaviour }\end{array}$ & Artifact & Dialogue & $\begin{array}{l}\text { Physical } \\
\text { behaviour }\end{array}$ & Artifact \\
\hline $\mathrm{D}$ & $\mathrm{N}$ & question & $\begin{array}{l}\text { current } \\
\text { context }\end{array}$ & But what's this door back there? & gesture & $\begin{array}{l}\text { handout } \\
\text { pictures }\end{array}$ \\
\hline $\mathrm{N}$ & $\mathrm{D}$ & explain & $\begin{array}{l}\text { current } \\
\text { context }\end{array}$ & $\begin{array}{l}\text { This one is another - so you've } \\
\text { basically got a T entrance }\end{array}$ & gesture & \\
\hline $\mathrm{D}$ & $\mathrm{N}$ & $\begin{array}{l}\text { request } \\
\text { clarification }\end{array}$ & $\begin{array}{l}\text { current } \\
\text { context }\end{array}$ & $\begin{array}{l}\text { So the reason somebody from the } \\
\text { front can't come around in the side } \\
\text { door is because there is that round } \\
\text { table on the side - }\end{array}$ & gesture & $\begin{array}{l}\text { handout } \\
\text { pictures }\end{array}$ \\
\hline $\mathrm{N}$ & $\mathrm{D}$ & $\begin{array}{l}\text { clarify, } \\
\text { explain }\end{array}$ & $\begin{array}{l}\text { current } \\
\text { context } \\
\text { current } \\
\text { context }\end{array}$ & $\begin{array}{l}\text { Well then I am going to have to walk } \\
\text { all the way around and come in the } \\
\text { side. I don't want to do that }\end{array}$ & gesture & \\
\hline $\mathrm{D}$ & $\mathrm{N}$ & $\begin{array}{l}\text { request } \\
\text { clarification }\end{array}$ & $\begin{array}{l}\text { current } \\
\text { context }\end{array}$ & $\begin{array}{l}\text { That's not the area where the table } \\
\text { is? }\end{array}$ & gesture & \\
\hline $\mathrm{N}$ & $\mathrm{D}$ & clarify & $\begin{array}{l}\text { current } \\
\text { context }\end{array}$ & $\begin{array}{l}\text { There's no access to any of this from } \\
\text { that area }\end{array}$ & & \\
\hline $\mathrm{D}$ & $\mathrm{N}$ & agree & & Right, right & $\begin{array}{l}\text { gesture } \\
\text { write on }\end{array}$ & $\begin{array}{l}\text { personal } \\
\text { notes }\end{array}$ \\
\hline $\mathrm{N}$ & $\mathrm{D}$ & explain & $\begin{array}{l}\text { current } \\
\text { context }\end{array}$ & $\begin{array}{l}\text { So basically, where are we again } \\
\text { here? Ok, where, there is the med } \\
\text { room, so there's no entrance, so if } \\
\text { I'm here and I want to get a sheet } \\
\text { back here l'm coming in this room, } \\
\text { I'm not going all the way around } \\
\text { there or all the way around here... }\end{array}$ & gesture & $\begin{array}{l}\text { handout } \\
\text { floor } \\
\text { plan }\end{array}$ \\
\hline
\end{tabular}




\section{Table 13.}

Example of coded exchanges

\begin{tabular}{|c|c|c|c|c|c|c|c|}
\hline Speaker & Receiver & $\begin{array}{l}\text { Verbal } \\
\text { behaviour }\end{array}$ & Artifact & Content & $\begin{array}{l}\text { Physical } \\
\text { behaviour }\end{array}$ & Artifact & Exchange \\
\hline $\mathrm{D}$ & $\mathrm{N}$ & suggest & plan & $\begin{array}{l}\text { If you were going to - } \\
\text { so designers we do } \\
\text { everything visually } \\
\text { right, could you } \\
\text { highlight the most used } \\
\text { and the least used areas } \\
\text { on here }\end{array}$ & gesture & $\begin{array}{l}\text { handout } \\
\text { floor } \\
\text { plan }\end{array}$ & MGMT \\
\hline $\mathrm{N}$ & D & $\begin{array}{l}\text { evaluate, } \\
\text { explain }\end{array}$ & $\begin{array}{l}\text { plan, } \\
\text { current } \\
\text { context }\end{array}$ & $\begin{array}{l}\text { Well the problem is } \\
\text { there is are two levels } \\
\text { right, there is level one } \\
\text { and level two, because } \\
\text { the counter space here } \\
\text { is even less used than } \\
\text { some of the shelf space, } \\
\text { because the area }\end{array}$ & & & INFO \\
\hline D & $\mathrm{N}$ & $\begin{array}{l}\text { request } \\
\text { clarification }\end{array}$ & $\begin{array}{l}\text { current } \\
\text { context }\end{array}$ & There's shelving here? & sketch & $\begin{array}{l}\text { handout } \\
\text { floor pla. }\end{array}$ & \\
\hline $\mathrm{N}$ & $\mathrm{D}$ & clarify & $\begin{array}{l}\text { current } \\
\text { context }\end{array}$ & $\begin{array}{l}\text { There are shelves here } \\
\text { with the patient in room } \\
\text { bins are all along, so } \\
\text { the area where patient } \\
\text { one's bins are there are } \\
\text { four rooms over here } \\
\text { but then there are } \\
\text { individual rooms }\end{array}$ & gesture & $\begin{array}{l}\text { handout } \\
\text { floor } \\
\text { plan }\end{array}$ & \\
\hline $\mathrm{D}$ & $\mathrm{N}$ & order & & $\begin{array}{l}\text { So number one then, } \\
\text { you can have three or } \\
\text { four areas }\end{array}$ & & & MGMT \\
\hline
\end{tabular}

Table 14.

Example dialogue showing suggestion and evaluation exchanges

\begin{tabular}{|c|c|c|c|c|c|}
\hline Speaker & Receiver & $\begin{array}{l}\text { Verbal } \\
\text { behaviour }\end{array}$ & Artifact & Dialogue & Exchange \\
\hline $\mathrm{D}$ & Team & $\begin{array}{l}\text { request } \\
\text { clarification, } \\
\text { suggest }\end{array}$ & $\begin{array}{l}\text { current } \\
\text { context } \\
\text { idea }\end{array}$ & $\begin{array}{l}\text { "And there is just too many permutations that it } \\
\text { is not practical to have something that } \\
\text { standardizes? - like a bar dispenser so its like } 1 \\
\text { shot of vodka, } 2 \text { shots of vodka... }\end{array}$ & ID \\
\hline N1 & $\mathrm{D}$ & doubt & idea & $\begin{array}{l}\text { I think you'd get into problems with control in } \\
\text { that sense, depending on if you lock it up - its } \\
\text { not a terrible idea }\end{array}$ & EVAL \\
\hline $\mathrm{D}$ & Team & clarify & idea & Pretend its not an issue with security & \\
\hline $\mathrm{N} 2$ & Team & agree & idea & It's a good idea actually & \\
\hline N1 & $\mathrm{N} 2, \mathrm{D}$ & doubt, clarify & idea & But I wonder about expiry dates, ... & \\
\hline
\end{tabular}

